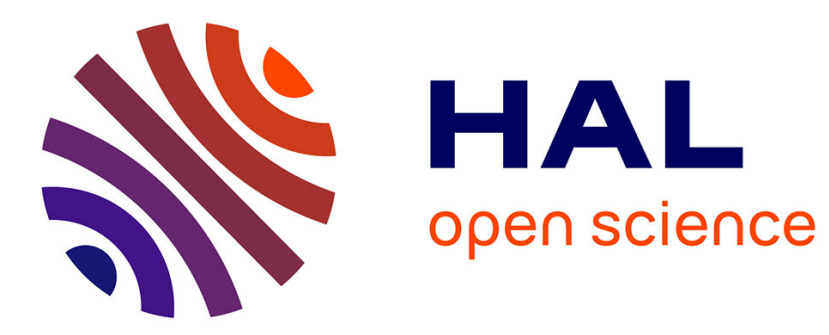

\title{
Enhanced sieving from exfoliated MoS2 membranes via covalent functionalization
}

\author{
Lucie Ries, Eddy Petit, Thierry Michel, Cristina Coelho Diogo, Christel \\ Gervais, Chrystelle Salameh, Mikhael Bechelany, Sébastien Balme, Philippe \\ Miele, Nicolas Onofrio, et al.
}

\section{To cite this version:}

Lucie Ries, Eddy Petit, Thierry Michel, Cristina Coelho Diogo, Christel Gervais, et al.. Enhanced sieving from exfoliated MoS2 membranes via covalent functionalization. Nature Materials, 2019, 18 (10), pp.1112-1117. 10.1038/s41563-019-0464-7 . hal-02310531

\section{HAL Id: hal-02310531 \\ https://hal.umontpellier.fr/hal-02310531}

Submitted on 23 Jan 2020

HAL is a multi-disciplinary open access archive for the deposit and dissemination of scientific research documents, whether they are published or not. The documents may come from teaching and research institutions in France or abroad, or from public or private research centers.
L'archive ouverte pluridisciplinaire HAL, est destinée au dépôt et à la diffusion de documents scientifiques de niveau recherche, publiés ou non, émanant des établissements d'enseignement et de recherche français ou étrangers, des laboratoires publics ou privés. 


\section{Enhanced sieving from exfoliated $\mathrm{MoS}_{2}$ membranes via covalent functionalization}

Lucie Ries, ${ }^{1}$ Eddy Petit, ${ }^{1}$ Thierry Michel, ${ }^{2}$ Cristina Coelho Diogo, ${ }^{3}$ Christel Gervais, ${ }^{4,5}$ Chrystelle Salameh, ${ }^{1}$ Mikhael Bechelany, ${ }^{1}$ Sébastien Balme, ${ }^{1}$ Philippe Miele, ${ }^{1,5}$ Nicolas Onofrio, ${ }^{6, *}$ Damien Voiry ${ }^{1, *}$

${ }^{1}$ Institut Européen des Membranes, IEM, UMR 5635, Université Montpellier, ENSCM, CNRS, 34095 Montpellier Cedex5, France.

${ }^{2}$ Laboratoire Charles Coulomb, L2C, Université de Montpellier, CNRS, 34095 Montpellier, France.

${ }^{3}$ Sorbonne Université, Institut des Matériaux de Paris Centre (IMPC), 4 place Jussieu, 75252 Paris cedex 05, France.

${ }^{4}$ Sorbonne Université, Collège de France, Laboratoire de Chimie de la Matière Condensée de Paris (LCMCP), 4 place Jussieu, 75252 Paris cedex 05, France.

${ }^{5}$ Institut Universitaire de France (IUF), MESRI, 1 rue Descartes, 75231 Paris cedex 05, France.

${ }^{6}$ Department of Applied Physics, The Hong Kong Polytechnic University, Hong Kong, China.

*Correspondence to: damien.voiry@umontpellier.fr; nicolas.onofrio@ polyu.edu.hk.

Nanolaminate membranes made of two-dimensional materials (2D) such as graphene oxide (GO) are promising candidates for molecular sieving via size-limited diffusion in the 2D capillaries, but high hydrophilicity makes these membranes unstable in water. Here, we report a nanolaminate membrane based on covalently functionalized molybdenum disulfide $\left(\mathrm{MoS}_{2}\right)$ nanosheets. The functionalized $\mathrm{MoS}_{2}$ membranes demonstrate $>90 \%$ and $\sim 87 \%$ rejection for micropollutants and $\mathrm{NaCl}$ respectively when operating under reverse osmotic conditions. The sieving performance and water flux of the functionalized $\mathrm{MoS}_{2}$ membranes are attributed to both control of the capillary widths of the nanolaminates and control of the surface chemistry of the nanosheets. We identified small hydrophobic 
functions, such as methyl, as the most promising functional group for water purification. Methyl functionalized nanosheets show higher water permeation rates as confirmed by our molecular dynamic simulations, while maintaining high $\mathrm{NaCl}$ rejection. Control of the surface chemistry and the interlayer spacing therefore offers opportunities to tune the selectivity of the membranes while enhancing their stability.

Membranes are semi-permeable barriers that allow separating contaminants depending on various parameters including their size, charge and chemical affinity. Exfoliated twodimensional materials have demonstrated promising performance as membranes for nanofiltration and desalination ${ }^{1-7}$. Seminal contributions from Nair's group have shown that stacking 2D materials such as graphene oxide can be used as nanolaminate membranes ${ }^{3,10}$. The pore size - dictated by the capillary width between the nanosheets - enables to efficiently sieve chemical species such as small ions and molecules depending on their size ${ }^{3,5}$. The remarkable property of the GO membranes is attributed to the unique surface chemistry of the nanosheets combining graphene-like and oxidized domains rich in oxygenated functional groups ${ }^{6,10-12}$. The graphene domains of graphene oxide nanosheets enable fast water diffusion thanks to large slip length whereas the oxygenated functions play the role of spacers allowing water to penetrate in the $2 \mathrm{D}$ capillaries ${ }^{12,13}$. Unfortunately the oxygenated functional groups render GO nanosheets highly hydrophilic which causes uncontrollable swelling of the membranes ${ }^{1}$. The swelling of GO membranes currently limits the control of its porosity - dramatically limiting the sieving performance - and eventually leads to irreversible degradation of the membrane integrity. Other 2D materials such as transition metal dichalcogenides have been investigated as nanolaminate membranes ${ }^{12,13,6,14,15}$. In particular $\mathrm{MoS}_{2}$ nanosheets have been explored as building blocks of 
membranes for water purification and gas sieving ${ }^{14,16}$. However the use of $\mathrm{MoS}_{2}$ remains strongly hampered by the difficulty in controlling the width of the capillaries that is found to be critical for the sieving performance ${ }^{14}$.

\section{Preparation and characterization of the functionalized $\mathrm{MoS}_{2}$ membranes}

We fabricated nanolaminate membranes consisting in exfoliated single-layer nanosheets of $\mathrm{MoS}_{2}$ functionalized with different types of functional groups varying their hydrophilic character. Single layer nanosheets of $\mathrm{MoS}_{2}$ were obtained following our previously reported method based on lithium intercalation ${ }^{17,18}$. Covalent functionalization of the $\mathrm{MoS}_{2}$ nanosheets was carried out by reacting the exfoliated nanosheets with organohalide molecules ${ }^{19}$. We selected 3 types of organohalide molecules: iodomethane, 2-iodoethanol and 2-iodoacetamide in order to vary the polarity of the grafted functional groups. The obtained functionalized $\mathrm{MoS}_{2}$ membranes will be now referred as acetamide-, methyl-, and ethyl-2-ol- functionalized $\mathrm{MoS}_{2}$ membranes (Supplementary Fig. 4) ${ }^{20}$. The single-layer $\mathrm{MoS}_{2}$ nanosheets were vertically stacked using vacuum filtration ${ }^{3,8,21,22}$. A typical functionalized $\mathrm{MoS}_{2}$ membrane is shown in Figure 1a,b. The high stability of the $\mathrm{MoS}_{2}$ nanosheets in solution translates into highly uniform membranes - no pinholes can be found under scanning electron microscope (SEM) (Figure 1c). The cross-section view of the membrane using SEM suggests a high degree of alignment of the nanosheets in the vertical direction (Figure 1d, Supplementary Fig. 5). High-resolution TEM images show the crystalline nature of the functionalized nanosheets (Figure 1e), while elemental mapping confirms the presence of functional groups on the surface of the nanosheets (Figure 1f). The covalent nature of the attachment of the functional groups was demonstrated by infra-red spectroscopy (Supplementary Figs. 7,8). ${ }^{13} \mathrm{C}$ CP MAS NMR was used to qualitatively assess the 
covalent attachment of the functional groups by comparing the chemical shift of the aliphatic carbon $(\alpha-C)$ of the functional group before and after the reaction - the spectra from the organohalide molecules are presented in Supplementary Figure 10. The chemical shift from the $\alpha-C$ of the grafted molecules appears at $50.0 \mathrm{ppm}, 34.7 \mathrm{ppm}$ and $49.4 \mathrm{ppm}$ for acetamide-, methyl- and ethyl-2-ol- functionalized $\mathrm{MoS}_{2}$ respectively compared to $-2.5 \mathrm{ppm},-23.2 \mathrm{ppm}$ and $9 \mathrm{ppm}$ for the corresponding $\alpha-\mathrm{C}$ of the organohalide references (Fig. 2a) ${ }^{19}$. The extent of functions grafted on the nanosheets was estimated using X-Ray photoelectron spectroscopy (XPS) and the density of functional groups reaches $\sim 40$ at.\% corresponding to a density of functional groups of $\sim 4.6 \times 10^{14} \mathrm{~cm}^{-2}$ (Supplementary Figs. 9-12 and Table 2).

10 We investigated the structure of the functionalized $\mathrm{MoS}_{2}$ membranes using X-ray diffraction (XRD). In the case of $\mathrm{MoS}_{2}$ membranes, an intense peak can be detected at $\sim 13.9^{\circ}$ and attributed to the (002) peak corresponding to interlayer spacing (d) of $\sim 6.18 \AA$ (Figure $2 \mathrm{~b}, \mathrm{c}$ ). The (002) peak is slightly shifted compared to the position from bulk $2 \mathrm{H}-\mathrm{MoS}_{2}$ crystal confirming the quasi-perfect restacking of the nanosheets (Supplementary Fig. 14). After functionalization, the $(002)$ peak at $\sim 13.9^{\circ}$ is displaced to lower angles at $\sim 8.8^{\circ}, \sim 7.5^{\circ}$, and $~$ $8.2^{\circ}$ for acetamide-, methyl- and ethyl-2-ol- functionalized $\mathrm{MoS}_{2}$ respectively (Figure 2b). These displacements correspond to the increase of the interlayer spacing due to the presence of the grafted functions (Figure 2d). It is worth noting that the (002) peak from pristine $\mathrm{MoS}_{2}$ completely disappears after functionalization suggesting that all the nanosheets in the membrane are functionalized. According to XRD and assuming a thickness of $6.15 \AA$ for a single layer of $\mathrm{MoS}_{2}$ - including the van der Waals radius of two sulfur atoms [See "X-Ray diffraction (XRD) on $\mathrm{MoS}_{2}$ membranes" in the Supplementary materials] - the width of the capillary $(\delta)$ of the $\mathrm{MoS}_{2}$ membranes varies from $\sim 0.03 \AA$ for non-functionalized $\mathrm{MoS}_{2}$ up to $\sim 5.4 \AA$ for methyl- 
functionalized $\mathrm{MoS}_{2}$ (Figure 2b-e, Supplementary Table 3). One of the main limitation of graphene oxide membranes is the uncontrollable swelling when immerged in water, which deteriorates the sieving performance towards smaller chemical species and ions ${ }^{1,23}$. The functionalized $\mathrm{MoS}_{2}$ membranes demonstrate limited swelling below than $<15 \%$ after 15 hours in water compared to $\sim 65 \%$ for graphene oxide (Figure 2e,f, Supplementary Figs. 16-18). This shows that covalent functionalization of the $\mathrm{MoS}_{2}$ membranes increases the width of the capillaries while limiting the swelling. The effect of the functionalization of the $\mathrm{MoS}_{2}$ nanosheets can directly be observed by contact angle measurements. Figure $2 \mathrm{~g}$ shows the contact angle of a water droplet on pristine $\mathrm{MoS}_{2}$ membranes compared to their functionalized counterparts. As expected, the contact angle increases with the hydrophobic nature of the grafted moieties and varies from $\sim 58^{\circ}$ up to $90^{\circ}$ for pristine and methyl-functionalized $\mathrm{MoS}_{2}$ respectively in perfect agreement with the decrease of the polarity of the functional groups.

\section{Ionic and water transport in the nanolaminate membranes}

We first evaluate the ionic transport across the functionalized $\mathrm{MoS}_{2}$ membranes for various salts: $\mathrm{LiCl}, \mathrm{NaCl}$ and $\mathrm{KCl}$ using two-compartment cells filled with a salt solution and deionized water [see "Ion permeance measurements" section in the Supplementary Information]. The functionalized membranes demonstrate low permeation rates for $\mathrm{Li}^{+}, \mathrm{Na}^{+}$and $\mathrm{K}^{+}$(Figure 3a,b). The ion permeation rates for acetamide- functionalized membranes are found to strictly depend on the ionic strength, while the permeation rates for ethyl-2-ol- functionalized $\mathrm{MoS}_{2}$ membranes do not significantly change with the increase of the ion concentration. At $1 \mathrm{M}$ of $\mathrm{NaCl}-$ corresponding to ionic strength of $1 \mathrm{M}-$ the permeation rates for $\mathrm{Na}^{+}$are estimated to $4.2 \times 10^{-5}$ $\left( \pm 8.1 \times 10^{-6}\right) \mathrm{mol} \mathrm{h}^{-1} \mathrm{~m}^{-2}$ bar $^{-1}$ and $7.6 \times 10^{-6}\left( \pm 1.6 \times 10^{-6}\right) \mathrm{mol} \mathrm{h}^{-1} \mathrm{~m}^{-2}$ bar $^{-1}$ for acetamide- and 
ethyl-2-ol- functionalized $\mathrm{MoS}_{2}$ membranes respectively. These values are two orders of magnitude lower than the expected permeation rates obtained from the classical diffusion law (Supplementary Table 4) [See "Permeation rate calculations" section in the Supplementary Materials]. They are also 3 orders of magnitude lower than that of graphene oxide membranes $\left(5 \times 10^{-2} \mathrm{~mol} \mathrm{~h}^{-1} \mathrm{~m}^{-2} \mathrm{bar}^{-1}\right)$ and similar to the $\mathrm{Na}^{+}$permeation rates for graphene oxide/graphene membranes $\left(5 \times 10^{-5} \mathrm{~mol} \mathrm{~h}^{-1} \mathrm{~m}^{-2} \mathrm{bar}^{-1}\right)^{1}$. Such low permeation rates are attributed to the pore steric hindrance on hydrated ions as the hydrated ion diameters are typically larger than the capillary width of the functionalized $\mathrm{MoS}_{2}$ membranes (Supplementary Table 6). Other factors such as the electrostatic interactions and the binding energy are also found to influence of the ionic transport (Supplementary Tables 5,6 and Supplementary Figs. 20,21) ${ }^{5}$.

The effect of the nature of the functional groups on the water permeance of $\mathrm{MoS}_{2}$ membranes was then investigated in forward (FO) and reverse osmotic (RO) configurations. The thickness of the $\mathrm{MoS}_{2}$ membranes was precisely adjusted from $250 \mathrm{~nm}$ up to $2 \mu \mathrm{m}$ by controlling the volume of $\mathrm{MoS}_{2}$ solution to be filtered. When used as FO membranes [See "Forward osmosis-driven measurements" section in the Supplementary Materials], the flux of water reaches up to 0.15 $( \pm 0.04) \mathrm{L} \mathrm{m}^{-2} \mathrm{~h}^{-1}$ bar $^{-1}$ for ethyl-2-ol- functionalized $\mathrm{MoS}_{2}-2.5$ and 3-fold higher than that of coordinated GO and dye-decorated $\mathrm{MoS}_{2}$ nanosheets respectively (Supplementary Fig. 23) ${ }^{4,6}$. We tested the $\mathrm{MoS}_{2}$ nanolaminate membranes in RO configuration and the water flux was measured using pure water in a dead-end cell [see "Reverse osmosis (RO) measurements" section in the Supplementary Information]. Interestingly water does not flow across the nonfunctionalized $\mathrm{MoS}_{2}$ membranes (Figure 3c). This observation agrees with the close-to-ideal stacking of the nanosheets. In the case of non-functionalized $\mathrm{MoS}_{2}$, the pore size of the capillaries between two successive nanosheets is estimated to $\delta \sim 0.03 \AA$ - too small for letting 
water permeate ${ }^{14}$. At the opposite water permeates through all the functionalized $\mathrm{MoS}_{2}$ membranes. The highest water flux is obtained for ethyl-2-ol- functionalized membranes with a flux of $\sim 45 \mathrm{~L} \mathrm{~m}^{-2} \mathrm{~h}^{-1}$ bar $^{-1}$ for a thickness of $250 \mathrm{~nm}$ (Figure 3c). For comparison, the highest water flux reported for GO membrane typically remains below $10 \mathrm{~L} \mathrm{~m}^{-2} \mathrm{~h}^{-1}$ bar $^{-1}$ for membranes with similar thickness ${ }^{14}$. Interestingly we found that the nature of the functional groups has profound effect on the permeation rate. The water flux decreases with the polarity of the functional groups demonstrating the importance of controlling the surface chemistry of the nanosheets for enhancing the water flux across the membrane (Figure 3d). We estimated the $\mathrm{NaCl}$ rejection from the different functionalized membranes with thickness ranging from $250 \mathrm{~nm}$ up to $1000 \mu \mathrm{m}$ using a salt concentration of $0.58 \mathrm{wt} . \%(0.1 \mathrm{M})$. All the functionalized $\mathrm{MoS}_{2}$ membranes are found efficient towards the separation of $\mathrm{NaCl}$ from water and the salt rejection is maintained as high as $70 \%$ for ethyl-2-ol- functionalized $\mathrm{MoS}_{2}$ membranes with a thickness of $250 \mathrm{~nm}$ for a water flux of $43 \mathrm{~L} \mathrm{~m}^{-2} \mathrm{~h}^{-1} \mathrm{bar}^{-1}$ (Fig. 3c). Remarkably- the water permeation from methyl- functionalized $\mathrm{MoS}_{2}$ membranes is largely preserved for thicknesses up to $4 \mu \mathrm{m}$ and we measured a water flux of $24( \pm 0.6) \mathrm{L} \mathrm{m}^{-2} \mathrm{~h}^{-1}$ bar $^{-1}$ for $2 \mu \mathrm{m}$-thick membranes -14 times higher than ethyl-2-ol- functionalized $\mathrm{MoS}_{2}$ membranes with same thickness $\left(1.68 \mathrm{~L} \mathrm{~m}^{-2} \mathrm{~h}^{-1} \mathrm{bar}^{-1}\right)$ (Supplementary Fig. 25). The behavior of methyl-functionalized $\mathrm{MoS}_{2}$ membranes is in apparent contradiction with the expected exponential decrease of the flux as the thickness increases. This phenomenon is attributed to locally higher degree of functionalization of the nanosheets together with the hydrophobic nature of the methyl groups - responsible for its improved slip length [see "Influence of the membrane thickness on the functionalization degree and stacking order of the $\mathrm{MoS}_{2}$ nanosheets" and "Velocity profiles and slip length estimation" sections in the Supplementary Information] ${ }^{24-27}$. 


\section{Micropollutant removals and water desalination by reverse osmosis}

To further validate our approach, we assess the performance of functionalized $\mathrm{MoS}_{2}$ membranes for nanofiltration and desalination. We measured permeation rates for several micropollutants in RO dead-end configuration [See "Molecular sieving performances under reverse osmosis conditions" section in the Supplementary Information]. We selected four key micropollutants such as an organic dye: phtalocyanine, a psycho-active drug: caffeine, a common drug: acetaminophen, and a hormone: $\beta$-estradiol - for which strategies of elimination are scarce and costly. We note that the micropollutants possess a large range of size from $\sim 3.8 \AA$ up $\sim 10 \AA$ (Supplementary Table 7). Our measurements reveal that the rejection: $R\left(R=1-C_{\text {permeate }} / C_{\text {feed }}\right)$ for all micropollutants tested is higher than $90 \%$ while the water flux is maintained above $3 \mathrm{~L} \mathrm{~m}^{-2} \mathrm{~h}^{-1}$ $\operatorname{bar}^{-1}$ (Figure 3e). Notably, methyl- functionalized membranes are the most promising candidates towards water nano-decontamination; as the rejection reaches $\sim 93 \%$ and $\sim 94 \%$ for caffeine and acetaminophen respectively $100 \%$ for the organic dye and $\beta$-estradiol. We also tested the performance of membranes towards desalination in RO conditions in dead-end configuration under a pressure of 9 bar and a $0.58 \mathrm{wt} \% \mathrm{NaCl}$ solution [See "Desalination performance under reverse osmosis conditions" section in the Supplementary Information]. The salt rejection peaks at $87 \%$ with a water flux of $1.6 \mathrm{~L} \mathrm{~m}^{-2} \mathrm{~h}^{-1}$ bar $^{-1}$ for ethyl-2-ol- functionalized $\mathrm{MoS}_{2}$ membranes (Fig. 3f, Supplementary Table 8). Higher water fluxes are obtained using acetamidefunctionalized $\mathrm{MoS}_{2}$ membranes, for which an $82 \% \mathrm{NaCl}$ rejection and a water flux of $\sim 33.7 \mathrm{~L}$ $\mathrm{m}^{-2} \mathrm{~h}^{-1}$ bar $^{-1}$ is achieved. The desalination performances from the functionalized $\mathrm{MoS}_{2}$ membranes are superior to previous reports for $1 \mathrm{D}$ and $2 \mathrm{D}$ nanolaminate $\mathrm{RO}$ membranes. For instance, graphene oxide $\mathrm{RO}$ membranes exhibit a $\mathrm{NaCl}$ rejection of $\sim 40 \%$ with a water flux of 
$4 \mathrm{~L} \mathrm{~m}^{-2} \mathrm{~h}^{-1}$ bar $^{-1}$ when using a salt concentration of $0.12 \mathrm{wt} . \%$ and under 5 bar $^{5}$. Our results also compare favorably to the performance of nanolaminate RO membranes based on graphene oxide (GO)/graphene hybrids that demonstrate $\mathrm{a} \sim 75 \% \mathrm{NaCl}$ rejection with a water flux of $<0.46 \mathrm{~L} \mathrm{~m}^{-}$ ${ }^{2} \mathrm{~h}^{-1}$ bar $^{-1}$ when using a 0.58 wt.\% $\mathrm{NaCl}$ solution (Supplementary Table 9$)^{28}$. The functionalized $\mathrm{MoS}_{2}$ RO membranes also outperform hybrid GO/GO quantum dots and GO/carbon nanotubes RO membranes that exhibit $\mathrm{NaCl}$ rejections of $15 \%$ and $59 \%$ for water fluxes of $128 \mathrm{~L} \mathrm{~m}^{-2} \mathrm{~h}^{-1}$ bar $^{-1}$ and $5.5 \mathrm{~L} \mathrm{~m}^{-2} \mathrm{~h}^{-1}$ bar $^{-1}$ for a $\mathrm{NaCl}$ concentration of 0.02 and $0.06 \mathrm{wt} . \%$ respectively $\left(\right.$ Supplementary Table 9) ${ }^{29,30}$.

\section{Molecular dynamic simulations of water flux}

In a view to rationalize the experimental results, we performed classical molecular dynamics simulations of water transport in the different functionalized $\mathrm{MoS}_{2}$ nanochannels [see "Molecular dynamics simulations" section in the Supplementary Information]. To describe with precision the potential chemistry between water, functional groups and $\mathrm{MoS}_{2}$, including partial charge transfer and polarization effects, we performed reactive simulations ${ }^{29}$. Simulation cells consist in two water reservoirs separated by a nanochannel, and the external pressure is controlled using the fluctuating wall method ${ }^{30}$. A snapshot of a typical simulation cell is presented in Figure 4a. We first study the water flux inside pristine $\mathrm{MoS}_{2}$ as a function of channel width. Within the timescale of MD simulations, we did not observe any water molecule penetrating inside a $\mathrm{MoS}_{2}$ channel of $8 \AA$ width. When increasing the capillary width, the predicted water fluxes quickly rise to $1.1,5.3,110.5,499.0,1241.0$ and $3302.0 \mathrm{~L} \mathrm{~m}^{-2} \mathrm{~h}^{-1} \mathrm{bar}^{-1}$ for spacings of 10, 12, 14, 16, 18 and $20 \AA$, respectively (Figure $4 b$ ). The water flux was evaluated for the different types of $\mathrm{MoS}_{2}$ membranes at a fixed interlayer spacing of $12 \AA$ (Figure $4 \mathrm{f}$ ). We 
note that such value of interlayer spacing width is larger than that measured experimentally for non-functionalized $\mathrm{MoS}_{2}$ membranes. Fluxes predicted by MD simulation reach 5.3, 13.0, 1.9 and 3.2 $\mathrm{L} \mathrm{m}^{-2} \mathrm{~h}^{-1}$ bar $^{-1}$ for pristine $\mathrm{MoS}_{2}$, methyl-, acetamide- and ethyl-2-ol- functionalized $\mathrm{MoS}_{2}$ membranes in agreement with the experimental trend presented in Figure $3 \mathrm{c}$. To understand the effect of functionalization on the water flux, we studied the organization of water molecules in the various $\mathrm{MoS}_{2}$ nanochannels, under pressure. We computed density profiles and axial density function (ADF) of water confined in the channels. [See "Density profiles" and "Axial density function" section in the Supplementary Information]. For pristine channels, we found that water molecules are highly organized in layers (Supplementary Fig. 37) and along the flux direction with a period of $\sim 3 \AA$ (Fig. 4c). Interestingly water molecules typically sit on top of sulfur atoms (Figure 4c,d). Such organization of confined water is in agreement with recent simulations of water between $\mathrm{MoS}_{2}{ }^{33}$. ADF of water molecules between methyl-functionalized $\mathrm{MoS}_{2}$ shows minimal structure along the flux direction while no structure is observed between acetamide and ethyl-2-ol functionalized layers (Figure 4d). We further calculated the hydrogen bond correlation function ${ }^{34}$. We found lifetimes of $\sim 1$ ns for water inside $12 \AA \mathrm{MoS}_{2}$ capillaries, two orders of magnitude larger than in bulk water and characteristic of an ice-like structure. The presence of non-polar functional groups such as methyl lowers lifetimes by a factor of 2 leading to more bulk-like water (Supplementary Fig. 39). To correlate the water dynamics to the nature of the nanochannel, we analyzed the velocity of water molecules in contact with pristine and methyl-functionalized $\mathrm{MoS}_{2}$. Figure $4 \mathrm{e}$ displays the time-averaged water velocity map in the XZ plane (under constant pressure) and its projection along the channel direction (Supplementary Figs. 40,41). The positions of sulfur atoms are indicated by white and black dashed lines in the velocity map and its projection, respectively. 
We predict smaller velocity of water molecules located right under S-atoms. These locations represent "pinning points", electrostatic in nature, between highly polarized O-H and Mo-S bonds. This suggests that $\mathrm{S}$-atoms provide the template for the water structure. The presence of methyl groups substitutes some of these pinning points and enhances the velocity of water molecules located right below them. Our numerical simulations also reveal that methyl groups enhance the water flux by improving the water slip length and limiting the steric effect compared to other larger functional groups (Supplementary Figs. 31,42,43).

\section{Outlook}

We have shown that covalent functionalization of exfoliated nanosheets can efficiently control the interlayer spacing and enhance the sieving performance of the nanolaminate membranes. The functionalized $\mathrm{MoS}_{2}$ membranes demonstrate remarkable performance towards water purification and desalination compared to the current state of the art. Our experimental results suggest that small, hydrophobic functional groups such as methyl, are key for enhancing the water flow in $\mathrm{MoS}_{2}$ membranes as supported by our molecular dynamic simulations. We believe our strategy paves the way to the preparation of membranes with tunable sieving behaviour. The control of the surface chemistry of exfoliated 2D materials allows further exploring nanofluidic phenomena inside nanolaminate membranes at fundamental and practical levels for water purification or osmotic energy. 


\section{References}

1. Abraham, J. et al. Tunable sieving of ions using graphene oxide membranes. Nat. Nanotechnol. 12, 546-550 (2017).

2. Kim, H. W. et al. Selective Gas Transport Through Few-Layered Graphene and Graphene Oxide Membranes. Science 342, 91-95 (2013).

3. Joshi, R. K. et al. Precise and Ultrafast Molecular Sieving Through Graphene Oxide Membranes. Science 343, 752-754 (2014).

4. Chen, L. et al. Ion sieving in graphene oxide membranes via cationic control of interlayer spacing. Nature 550, 380-383 (2017).

5. Han, Y., Xu, Z. \& Gao, C. Ultrathin Graphene Nanofiltration Membrane for Water Purification. Adv. Funct. Mater. 23, 3693-3700 (2013).

6. Hirunpinyopas, W. et al. Desalination and Nanofiltration through Functionalized Laminar $\mathrm{MoS}_{2}$ Membranes. ACS Nano 11, 11082-11090 (2017).

7. Heiranian, M., Farimani, A. B. \& Aluru, N. R. Water desalination with a single-layer $\mathrm{MoS}_{2}$ nanopore. Nat. Commun. 6, 8616 (2015).

8. Nair, R. R., Wu, H. A., Jayaram, P. N., Grigorieva, I. V. \& Geim, A. K. Unimpeded permeation of water through helium-leak-tight graphene-based membranes. Science $\mathbf{3 3 5}$, 442-444 (2012).

9. Koltonow, A. R. \& Huang, J. Two-dimensional nanofluidics. Science 351, 1395-1396 (2016).

10. Wei, N., Peng, X. \& Xu, Z. Understanding Water Permeation in Graphene Oxide Membranes. ACS Appl. Mater. Interfaces 6, 5877-5883 (2014). 
11. Wei, N., Peng, X. \& Xu, Z. Breakdown of fast water transport in graphene oxides. Phys. Rev. E 89, 012113 (2014).

12. Sun, L., Huang, H. \& Peng, X. Laminar $\mathrm{MoS}_{2}$ membranes for molecule separation. Chem. Commun. 49, 10718-10720 (2013).

13. Sun, L. et al. Ultrafast Molecule Separation through Layered $\mathrm{WS}_{2}$ Nanosheet Membranes. ACS Nano 8, 6304-6311 (2014).

14. Wang, Z. et al. Understanding the Aqueous Stability and Filtration Capability of $\mathrm{MoS}_{2}$ Membranes. Nano Lett. 17, 7289-7298 (2017).

15. Deng, M., Kwac, K., Li, M., Jung, Y. \& Park, H. G. Stability, Molecular Sieving, and Ion Diffusion Selectivity of a Lamellar Membrane from Two-Dimensional Molybdenum Disulfide. Nano Lett. 17, 2342-2348 (2017).

16. Achari, A., S, S. \& Eswaramoorthy, M. High performance $\mathrm{MoS}_{2}$ membranes: effects of thermally driven phase transition on $\mathrm{CO}_{2}$ separation efficiency. Energy Environ. Sci. 9, 1224-1228 (2016).

17. Joensen, P., Frindt, R. F. \& Morrison, S. R. Single-layer $\mathrm{MoS}_{2}$. Mater. Res. Bull. 21, 457461 (1986).

18. Eda, G. et al. Photoluminescence from Chemically Exfoliated $\mathrm{MoS}_{2}$. Nano Lett. 11, 5111$5116(2011)$

19. Voiry, D. et al. Covalent functionalization of monolayered transition metal dichalcogenides by phase engineering. Nat. Chem. 7, 45-49 (2015).

20. Wang, H. et al. $\mathrm{MoSe}_{2}$ and $\mathrm{WSe}_{2}$ Nanofilms with Vertically Aligned Molecular Layers on Curved and Rough Surfaces. Nano Lett. 13, 3426-3433 (2013). 
21. Eda, G., Fanchini, G. \& Chhowalla, M. Large-area ultrathin films of reduced graphene oxide as a transparent and flexible electronic material. Nat. Nanotechnol. 3, 270-274 (2008).

22. Acerce, M., Voiry, D. \& Chhowalla, M. Metallic $1 \mathrm{~T}$ phase $\mathrm{MoS}_{2}$ nanosheets as supercapacitor electrode materials. Nat. Nanotechnol. 10, 313-318 (2015).

23. Yeh, C.-N., Raidongia, K., Shao, J., Yang, Q.-H. \& Huang, J. On the origin of the stability of graphene oxide membranes in water. Nat Chem 7, 166-170 (2015).

24. Holt, J. K. et al. Fast Mass Transport Through Sub-2-Nanometer Carbon Nanotubes. Science 312, 1034-1037 (2006).

25. Secchi, E. et al. Massive radius-dependent flow slippage in carbon nanotubes. Nature 537, $210-213$ (2016).

26. Balme, S. et al. Unexpected ionic transport behavior on hydrophobic and uncharged conical nanopore. Faraday Discuss. 210, 69-85 (2018).

27. Balme, S. et al. Ionic transport through sub-10 nm diameter hydrophobic high-aspect ratio nanopores: experiment, theory and simulation. Sci. Rep. 5, 10135 (2015).

28. Morelos-Gomez, A. et al. Effective $\mathrm{NaCl}$ and dye rejection of hybrid graphene oxide/graphene layered membranes. Nat. Nanotechnol. 12, 1083-1088 (2017).

29. Han, Y., Jiang, Y. \& Gao, C. High-Flux Graphene Oxide Nanofiltration Membrane Intercalated by Carbon Nanotubes. ACS Appl. Mater. Interfaces 7, 8147-8155 (2015).

30. Zhao, G., Hu, R., Zhao, X., He, Y. \& Zhu, H. High flux nanofiltration membranes prepared with a graphene oxide homo-structure. J. Membr. Sci. 585, 29-37 (2019).

31. van Duin, A. C. T., Dasgupta, S., Lorant, F. \& Goddard, W. A. ReaxFF: A Reactive Force Field for Hydrocarbons. J. Phys. Chem. A 105, 9396-9409 (2001). 
32. Lupkowski, M. \& van Swol, F. Computer simulation of fluids interacting with fluctuating walls. J. Chem. Phys. 93, 737-745 (1990).

33. Kwac, K. et al. Multilayer Two-Dimensional Water Structure Confined in MoS 2 . J. Phys. Chem. C 121, 16021-16028 (2017).

34. Chandra, A. Effects of ion atmosphere on hydrogen-bond dynamics in aqueous electrolyte solutions. Phys. Rev. Lett. 85, 768 (2000).

\section{Acknowledgments}

L.R. acknowledges scholarship from the Graduate School "Ecole doctorale des Sciences Chimiques Balard, ED 459". D.V. acknowledges financial supports from "Project Axe Transverse Santé" and CNRS Cellule Energie exploratory project: "NANOSMO”. This project has also received partial funding from the European Research Council (ERC) under the European Union's Horizon 2020 research and innovation programme (grant agreement No 804320). The French Région Ile de France - SESAME program is acknowledged for financial support (700 MHz NMR spectrometer). We thank The Hong Kong Polytechnic University and the department of Applied Physics for the computational resources. Didier Cot and Erwan Oliviero are acknowledged for the electron microscopy. We thank Valérie Flaud and Léa Causse for the XPS and the ICP-OES measurements.

\section{Author Contributions}

D.V. conceived the idea, designed the experiments and wrote the manuscript. L.R. designed the experiments with D.V., fabricated the membranes and performed membrane characterizations 
and analyzed the results. L.R. and D.V. analyzed the data and wrote the manuscript. E.P. carried out HPLC and liquid NMR spectroscopy measurements. T.M. performed RAMAN measurements with L.R. and discussed the results with D.V. and L.R.. C.C.D. and C.G. performed ${ }^{13} \mathrm{C}$ CAS NMR spectroscopy measurements and C.S. discussed the results with D.V. and L.R.. S.B. and M.B. assisted L.R. on ionic permeation experiments and discussed the water permeation results. N.O. performed the Molecular dynamic simulations and wrote the manuscript with D.V. and L.R.. P.M. discussed the results with D.V. and L.R. All of the authors edited the manuscript before submission.

\section{Competing financial interests}

The authors declare no competing financial interests.

\section{Data availability}

The data that support the plots within this paper and other findings of this study are available from the corresponding authors upon reasonable request.

\section{Methods}

\section{Synthesis of exfoliated single-layer $\mathrm{MoS}_{2}$ nanosheets}

$\mathrm{MoS}_{2}$ nanosheets were prepared via lithium intercalation method as reported previously. $0.4 \mathrm{~g}$ of

20 bulk crystal of $\mathrm{MoS}_{2}$ was mixed with $4 \mathrm{~mL}$ of n-butyllithium in hexane and refluxed for $48 \mathrm{~h}$ under argon ${ }^{18}$. The intercalated powder was then recovered by vacuum filtration and washed several times with hexane $(4 \times 50 \mathrm{~mL})$. The intercalated powder was exfoliated in water at $1 \mathrm{mg} / \mathrm{mL}$ and sonicated for $1 \mathrm{~h}$ to facilitate the exfoliation. The solution was then centrifuged to 
remove all lithium cations as well as the non-exfoliated materials ${ }^{35}$. The single-layer nature of the exfoliated nanosheets has previously been characterized ${ }^{18}$.

\section{Functionalization of single-layer $\mathrm{MoS}_{2}$ nanosheets}

Single-layer $\mathrm{MoS}_{2}$ nanosheets were functionalized using organohalide reagents. Functionalization of the nanosheets was performed either in solution or directly on the membranes. In liquid phase, the reagents were added to the $\mathrm{MoS}_{2}$ solution with a 10 -fold excess. After $48 \mathrm{~h}$, the solution was filtered on the polymeric support in order to fabricate the functionalized membranes following the method described above. Alternatively $\mathrm{MoS}_{2}$ nanosheets were functionalized after the fabrication of the membranes by diffusing the reactant solution through the lamellar structure. The membranes made of $\mathrm{MoS}_{2}$-Acet. and $\mathrm{MoS}_{2}-\mathrm{EtOH}$ were functionalized in solution prior the fabrication of the nanolaminate membranes. $\mathrm{MoS}_{2}-\mathrm{Met}$. membranes were functionalized directly on the porous support after the filtration of the $\mathrm{MoS}_{2}$ nanosheets. The non-functionalized $\mathrm{MoS}_{2}$ solution was first filtered over a nylon membrane with 220-nm pore-size. After all the solution was filtered, the solution of organohalide reagents (for example iodomethane) - in 10-fold excess - dissolved in water:2-propanol (2:1 in volume) was carefully poured in the filtration funnel while the $\mathrm{MoS}_{2}$ membrane remains fully wet. The reagent solution was let to slowly diffuse through the $\mathrm{MoS}_{2}$ membrane for 48 hours after what the membrane was rinsed with 2-propanol $(4 \times 50 \mathrm{~mL})$, ethanol $(4 \times 50 \mathrm{~mL})$ and water $(4 \times 50 \mathrm{~mL})$.

\section{Fabrication of $\mathrm{MoS}_{2}$ membranes}

The $\mathrm{MoS}_{2}$ membranes were prepared by vacuum filtration as described previously ${ }^{36}$. Briefly, the solution of exfoliated single-layer $\mathrm{MoS}_{2}$ nanosheets was filtered over porous polymeric supports with $25 \mathrm{~nm}$ and $220 \mathrm{~nm}$ pore sizes. Examples of polymeric support are typically made of 
nitrocellulose, nylon, PVDF. In this study, we used Nylon supports with $200 \mathrm{~nm}$ pore size for testing all the $\mathrm{MoS}_{2}$-based membranes. The size of the filtration apparatus was $3.5 \mathrm{~cm}$ in diameter. The as-prepared $\mathrm{MoS}_{2}$ membranes were then cut to a size of approx. $1 \mathrm{~cm}^{2}$ in order to confirm the reproducibility of the performance for the same batch. At least 4 membranes were tested for each batch. We also fabricated and tested $\mathrm{MoS}_{2}$ membranes from different batches in order to confirm the reproducibility of the results.

We measured that the density of the $\mathrm{MoS}_{2}$ membranes is $\sim 5.2 \mathrm{~g} \mathrm{~cm}^{-3}$. The porosity of the $\mathrm{MoS}_{2}$ membranes was estimated using the equation:

$$
\phi=1-\frac{\rho_{\text {membranes }}}{\rho_{\text {Mos }}}(1)
$$

where $\rho_{\text {membranes }}$ and $\rho_{M o S_{2}}$ are the density of the membrane and bulk $\operatorname{MoS}_{2}\left(5.09 \mathrm{~g} \mathrm{~cm}^{-3}\right)$, respectively. The density of the non-functionalized $\mathrm{MoS}_{2}$ membranes varies of only $5 \%$ compared to the density of bulk crystal. This indicates that the turbostatic stacking of the $\mathrm{MoS}_{2}$ nanosheets in the membrane is close to the stacking of $2 \mathrm{H} \mathrm{MoS}_{2}$ crystals suggesting a dense structure.

\section{Molecular dynamics simulations}

Simulations cells were built starting from a 6-atoms orthogonal primitive cell of $\mathrm{MoS}_{2}$ in the 1T' phase ${ }^{37}$ and replicated $20 \times 8$ in the in-plane directions to create one of the (upper and lower) channel wall. The curved region was achieved by bending to $90^{\circ} 4$ unit cells symmetrically along the long direction. This geometry insured a faster filling of the water molecule inside the channel. To further speed-up filling, water molecules were introduced using VMD ${ }^{38}$ in both reservoirs and inside the nanochannel (for $\delta \geq 10 \AA$ ). C, H, O and N force field parameters were 
taken from Ref. 39 and combined with that of $\mathrm{MoS}_{2}$ from Ref. 40. We slightly modified the S-O van der Waals parameters to improve the water/MoS 2 interactions [see "Reactive MD and interatomic potential" in the Supplementary Information]. All simulations were performed with the large-scale MD package LAMMPS ${ }^{41}$ with a time step of $0.5 \mathrm{fs}$ in the NPT ensemble at 300 $\mathrm{K}$ and under 1 Atmosphere in the periodic directions. Periodic boundary conditions were applied along the directions perpendicular to the channel. Simulation cells were minimized and equilibrated for a short 5 ps run without external pressure followed by 500 ps under an applied pressure of $500 \mathrm{MPa}$ to reach steady-state.

\section{References}

35. Kappera, R. et al. Phase-engineered low-resistance contacts for ultrathin $\mathrm{MoS}_{2}$ transistors. Nat. Mater. 13, 1128-1134 (2014).

36. Acerce, M., Voiry, D. \& Chhowalla, M. Metallic 1T phase $\mathrm{MoS}_{2}$ nanosheets as supercapacitor electrode materials. Nat. Nanotechnol. 10, 313-318 (2015).

37. Voiry, D. et al. Covalent functionalization of monolayered transition metal dichalcogenides by phase engineering. Nat. Chem. 7, 45-49 (2015).

38. Humphrey, W., Dalke, A. \& Schulten, K. VMD: visual molecular dynamics. J. Mol. Graph. 14, 33-38 (1996).

39. Kim, S.-Y. \& van Duin, A. C. T. Simulation of Titanium Metal/Titanium Dioxide Etching with Chlorine and Hydrogen Chloride Gases Using the ReaxFF Reactive Force Field. J. Phys. Chem. A 117, 5655-5663 (2013).

40. Ostadhossein, A. et al. ReaxFF Reactive Force-Field Study of Molybdenum Disulfide (MoS 2). J. Phys. Chem. Lett. 8, 631-640 (2017).

41. Plimpton, S. Fast Parallel Algorithms for Short-Range Molecular Dynamics. J. Comput. Phys. 117, 1-19 (1995). 
Figure 1 | Nanolaminate membranes made of covalently functionalized $\mathrm{MoS}_{2}$ nanosheets. ab, Photographs of a functionalized $\mathrm{MoS}_{2}$ membrane prepared by vacuum filtration. c, Top view of a functionalized $\mathrm{MoS}_{2}$ membrane observed by scanning electron microscope (SEM). d, Side view of a functionalized $\mathrm{MoS}_{2}$ membrane observed by SEM showing the high degree of the alignment of the vertically stacked $\mathrm{MoS}_{2}$ nanosheets forming 2D capillaries. e, High-resolution Transmission Electron Microscope (TEM) images of $\mathrm{MoS}_{2}$ nanosheets functionalized with acetamide groups. Interlayer spacing of $\sim 10.1 \AA$ can be observed from vertically aligned nanosheets (left) whereas the basal plane of the nanosheets are found crystalline (right) as confirmed by the diffraction pattern (inset). f, Energy Dispersive X-ray mapping of the Mo (in red) and $\mathrm{N}$ (in blue) elements from acetamide- functionalized $\mathrm{MoS}_{2}$ nanosheets.

Figure 2 | Characterization of the functionalized $\mathrm{MoS}_{2}$ membranes. a, ${ }^{13} \mathrm{C} \mathrm{CP}$ MAS NMR spectra for the different covalently functionalized $\mathrm{MoS}_{2}$ nanosheets. The peaks corresponding to 15 the $\alpha-C$ of the functional groups are detected at $\sim 50 \mathrm{ppm}, 34.7 \mathrm{ppm}$ and $49.7 \mathrm{ppm}$ for acetamide, methyl- and ethyl-2-ol- functionalized $\mathrm{MoS}_{2}$ respectively. b, X-ray diffraction spectra from pristine non-functionalized $\mathrm{MoS}_{2}$ membranes (blue) and functionalized $\mathrm{MoS}_{2}$ membranes with acetamide ( $\mathrm{MoS}_{2}$-Acet.; black), methyl ( $\mathrm{MoS}_{2}$-Met.; green) and ethyl-2-ol (MoS 2 -EtOH; orange). After functionalization the (002) peaks of the $\mathrm{MoS}_{2}$ nanosheets appear shifted towards lower angles due to the presence of the functional groups. c-d, Schematics of restacked $\mathrm{MoS}_{2}$ nanosheets (c) and acetamide-functionalized $\mathrm{MoS}_{2}(\mathbf{d})$ with the interlayer spacing: $\mathrm{d}$ and the capillary width: $\delta$. e, Evolution of the capillaries width from the $\mathrm{MoS}_{2}$ membranes when immerged in water. $\mathbf{f}$, Swelling of the graphene oxide membranes (in red) compared to the $\mathrm{MoS}_{2}$ 
membranes. g, Contact angle measurements from $\mathrm{MoS}_{2}$ and functionalized $\mathrm{MoS}_{2}$ membranes. The contact angle can to be tuned from $58^{\circ}$ for pristine $\mathrm{MoS}_{2}$ up to $90^{\circ}$ for methylfunctionalized $\mathrm{MoS}_{2}$. All error bars correspond to standard deviation of at least 4 samples.

Figure 3 | Performance of the functionalized $\mathrm{MoS}_{2}$ membranes towards water purification and desalination. a,b Permeation rates for $\mathrm{Na}^{+}, \mathrm{Li}^{+}$and $\mathrm{K}^{+}$across acetamide- functionalized (a) and ethyl-2-ol- functionalized (b) $\mathrm{MoS}_{2}$ membranes measured for different ionic strengths under forward osmosis. Chloride was used as counter ion in all the cases. The permeation rates are relatively stable even for the highest ionic strength corresponding to $1 \mathrm{M}$ feed solutions. c, Water flux (dashed lines) and salt rejection (dotted lines) measured from $\mathrm{MoS}_{2}$ and functionalized$\mathrm{MoS}_{2}$ membranes under reverse osmosis. The water fluxes have been obtained in dead end configuration using pure water. High water flux is observed for all functionalized $\mathrm{MoS}_{2}$ membranes whereas the flux is suppressed in the absence of functional groups. d, Evolution of the water flux with the water contact angle of the functionalized membranes. e, Rejection of 15 different micropollutants measured for the functionalized $\mathrm{MoS}_{2}$ membranes measured under a pressure of 2 bars. f, Comparison of the desalination performance from 500 nm-thick functionalized $\mathrm{MoS}_{2}$ membranes measured at 9 bar using a 0.58 wt.\% $\mathrm{NaCl}$ solution with other membranes made of carbon nanotubes, graphene oxide and their hybrids as well as polymeric membranes based on Thin Film Composite (TFC) technology. All error bars correspond to 20 standard deviation of at least 4 samples. We note that the salt concentration may differ between the experiments. The direct comparison of the desalination performance of the $\mathrm{MoS}_{2}$ membranes with previous results from the literature can be found in Supplementary Table 9. 
Figure 4 | Molecular dynamics simulations of water transport in 2D nanochannels. a, Snapshot of a typical atomistic model used in the simulations with the $\mathrm{x}, \mathrm{y}$ and $\mathrm{z}$ axes. The fluctuating wall method is used to control pressure in the water reservoirs. Periodic boundary conditions are applied in the $y$ - and z-direction. b, Number of water molecules crossing the midpoint of the nanochannel as a function of simulation time for various interlayer spacings $(d)$. The logarithmic of the flux versus the interlayer spacing is represented in the inset. The water flux varies as the cubic power of the spacing. c, Snapshots zooming-in the nanochannels of various functionalized $\mathrm{MoS}_{2}$ nanosheets. d, Axial density function representing the probability of finding a water molecule from a reference water molecule. For pristine case, we can clearly identify the structure of water in the axial direction with a period of approximately $3 \AA$ while no clear structure is observed for the functionalized cases. e, Time averaged velocity map of the first layer of water molecules in the xz plane and its projection along the channel direction $\left(\mathrm{v}_{\mathrm{x}}\right)$. The velocity of water molecules is enhanced by the presence of methyl groups. f, Number of water molecules crossing the mid-point of the nanochannel as a function of simulation time for the 4 channels described in (c). The corresponding water fluxes are summarized in the inset. The fluxes are normalized to surface of the nanochannel. Methyl-functionalized $\mathrm{MoS}_{2}$ channel enhances water flux. 
a

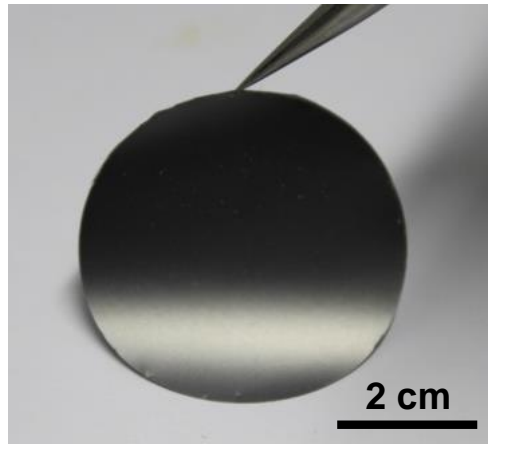

C

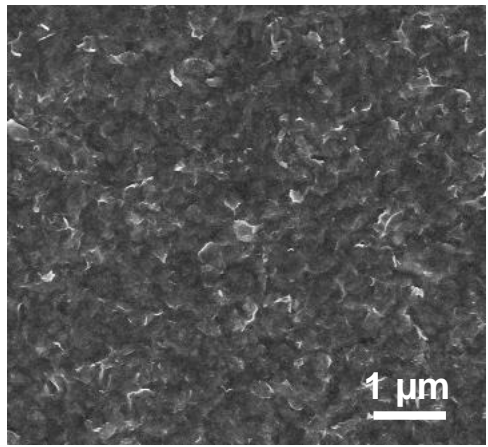

e

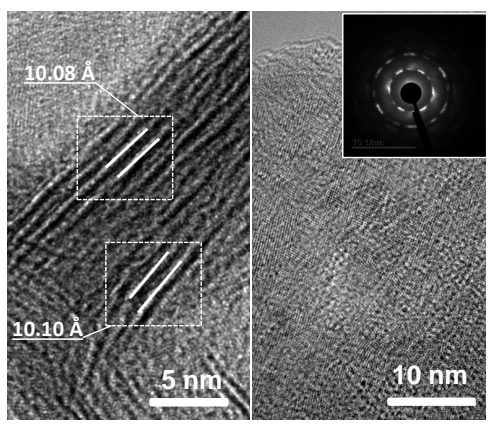

b

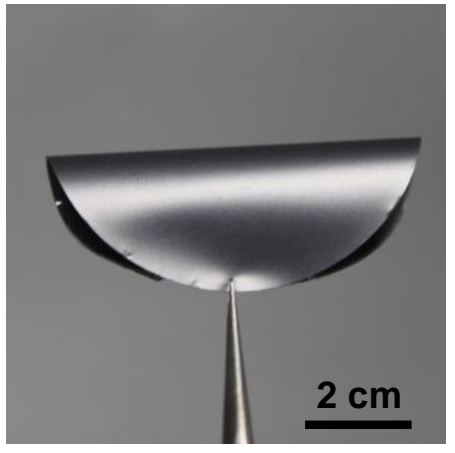

d

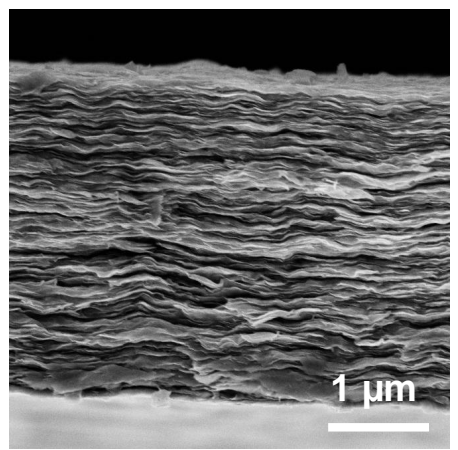

f

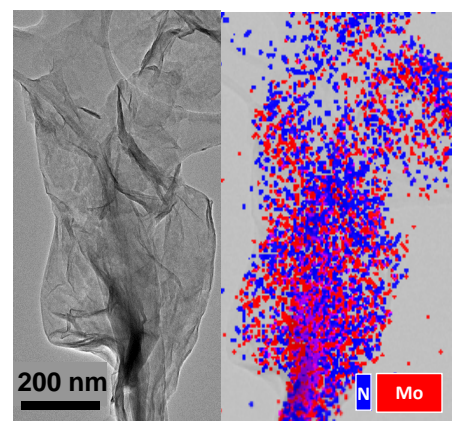


a

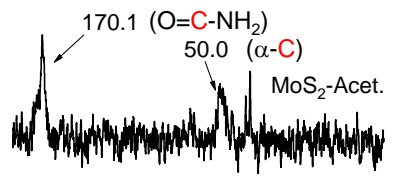

$(\alpha-C) 34.7$.

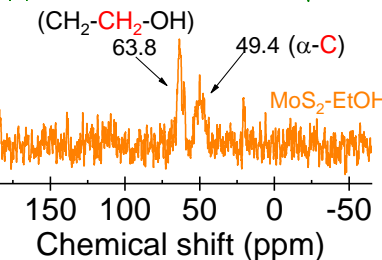

e

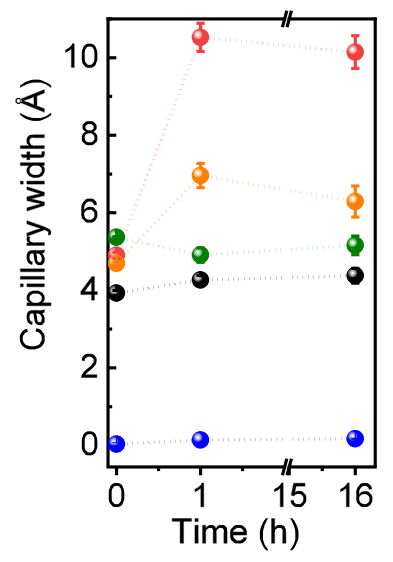

b

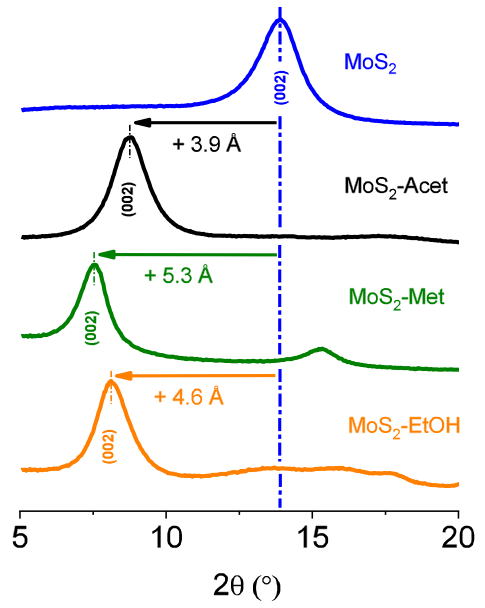

f

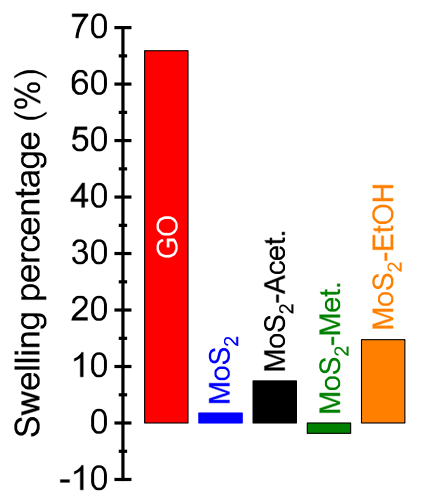

C

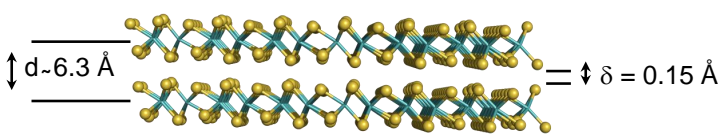

d

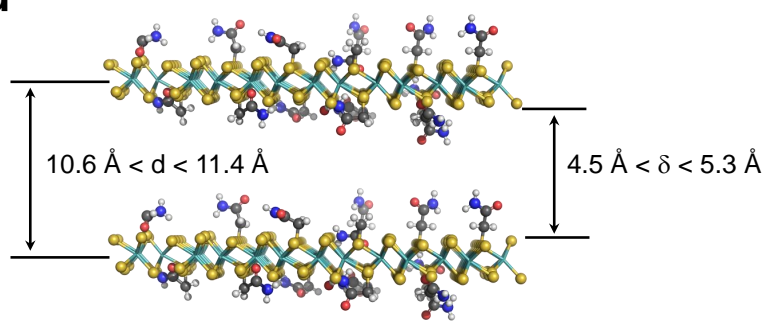

g

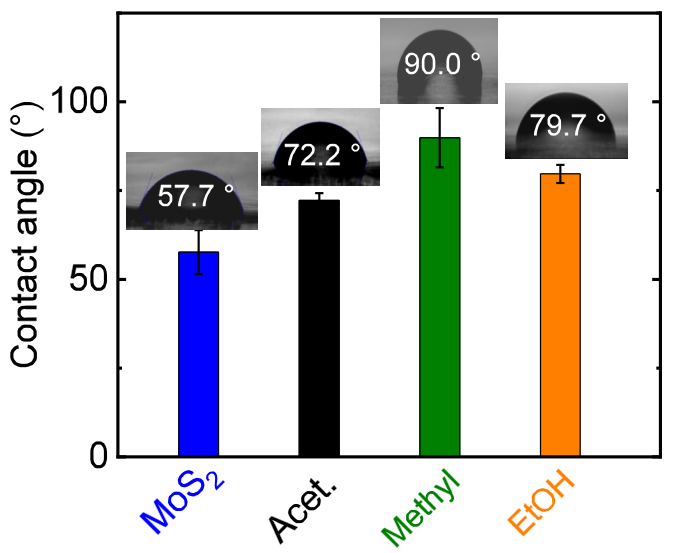


a

C

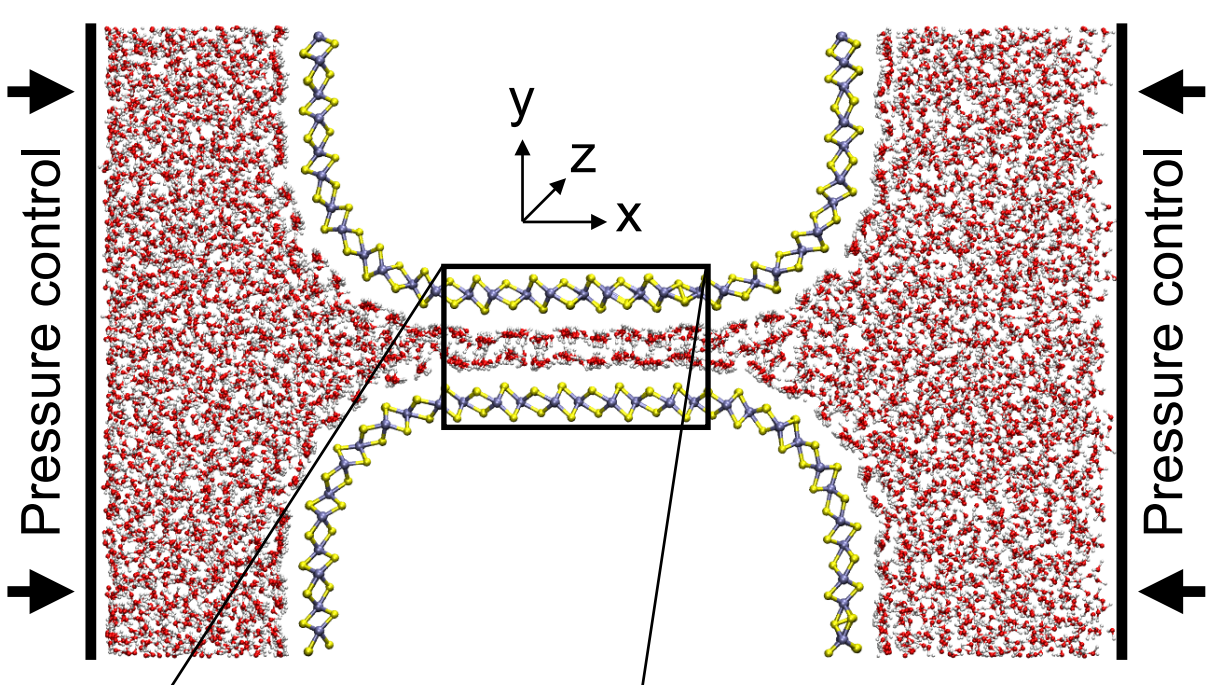

c

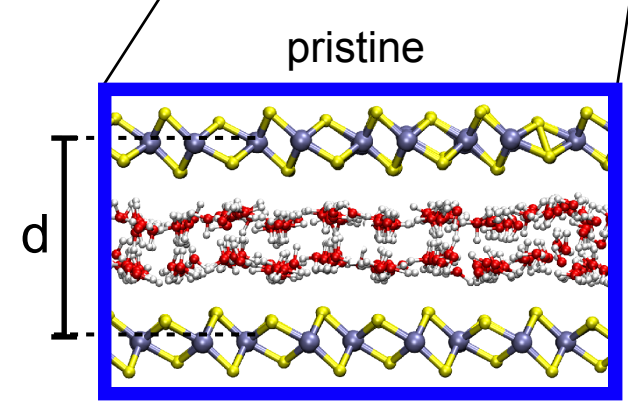

d

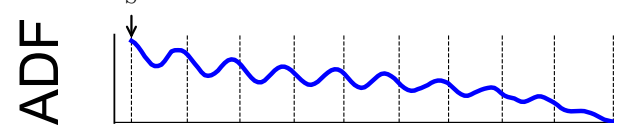

e

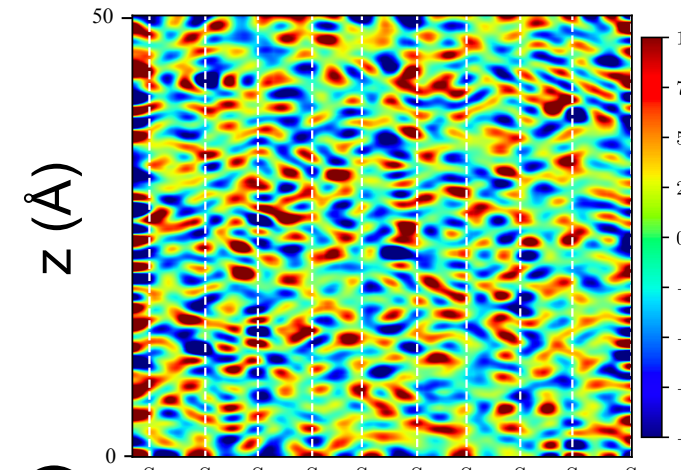

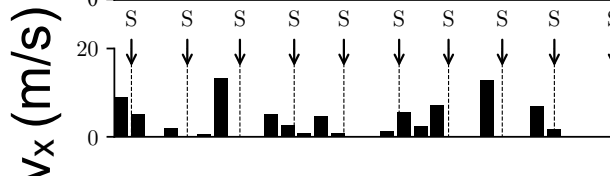

$x(\AA)$

methyl

$x(\AA)$ b
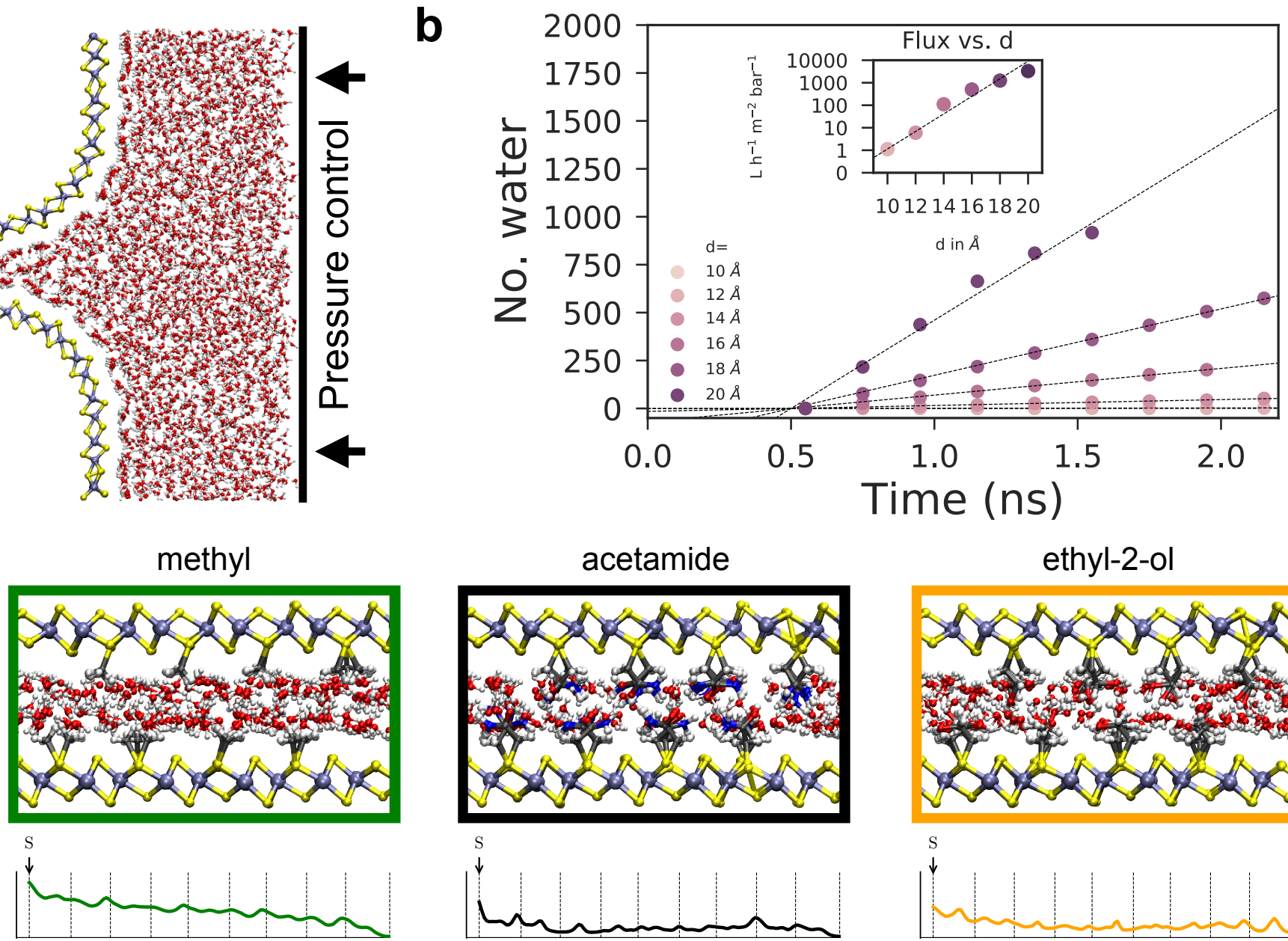

acetamide

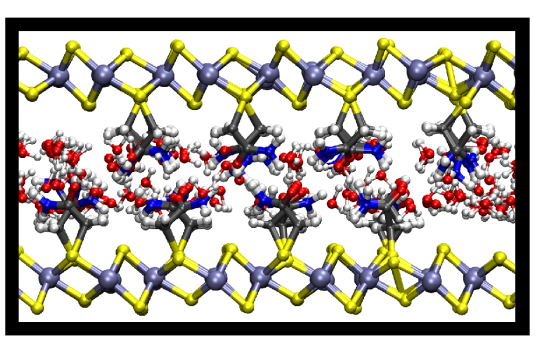

ethyl-2-ol
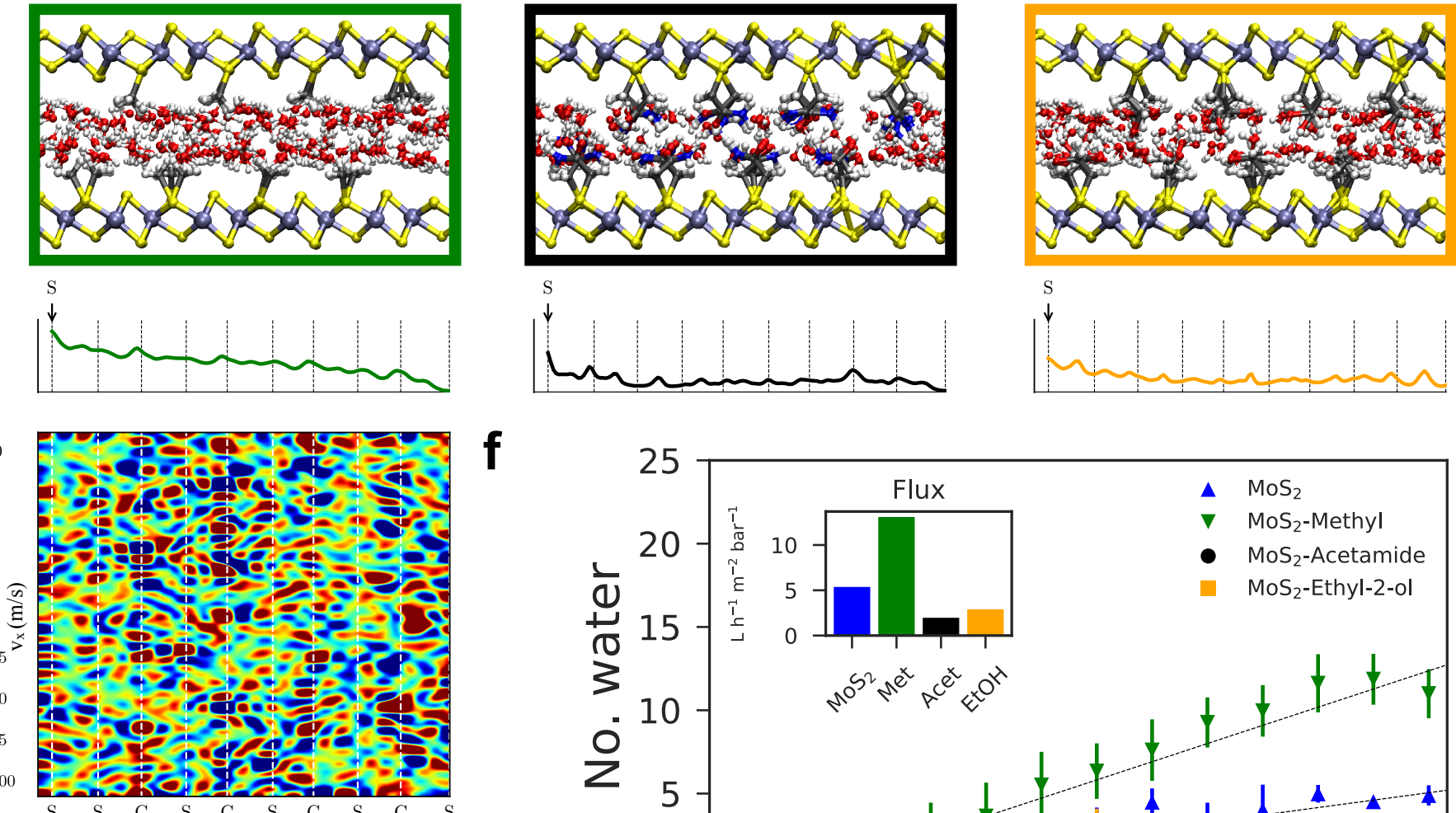

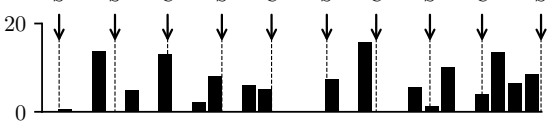

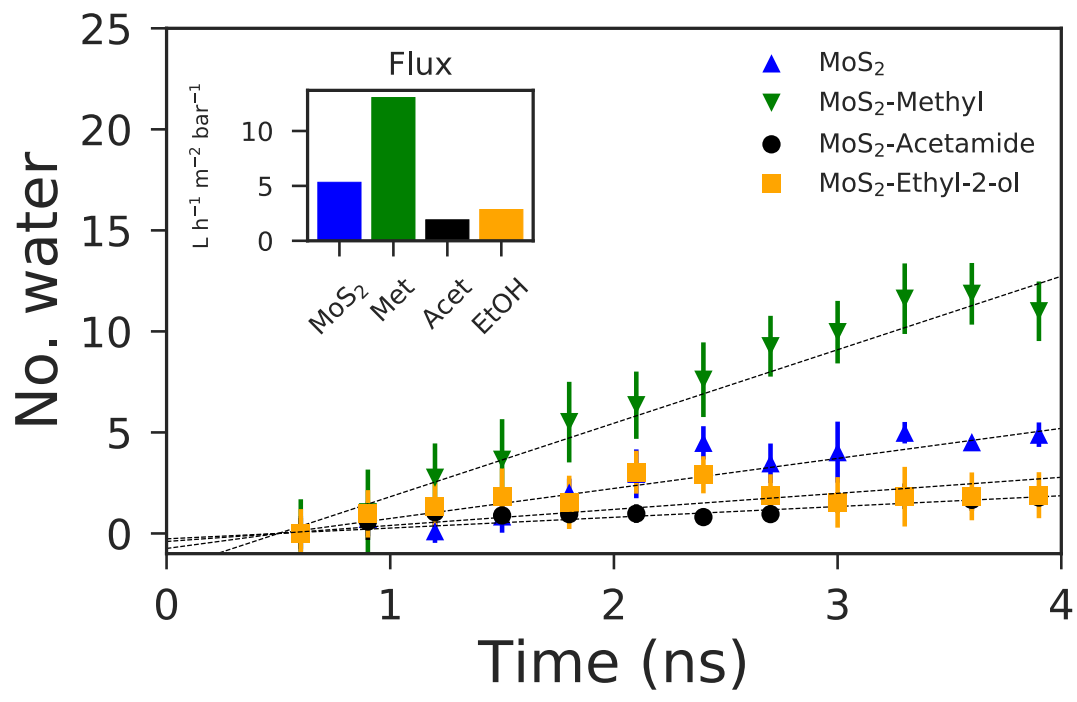


Supplementary Materials for

\section{Enhanced sieving from exfoliated $\mathrm{MoS}_{2}$ membranes via covalent functionalization}

Lucie Ries, ${ }^{1}$ Eddy Petit, ${ }^{1}$ Thierry Michel, ${ }^{2}$ Cristina Coelho Diogo,${ }^{3}$ Christel Gervais, ${ }^{4,5}$ Chrystelle Salameh, ${ }^{1}$ Mikhael Bechelany, ${ }^{1}$ Sébastien Balme, ${ }^{1}$ Philippe Miele, ${ }^{1,5}$ Nicolas Onofrio, ${ }^{6, *}$ Damien Voiry,

${ }^{1}$ Institut Européen des Membranes, IEM, UMR 5635, Université Montpellier, ENSCM, CNRS, 34095 Montpellier Cedex5, France.

2 Laboratoire Charles Coulomb, L2C, Université de Montpellier, CNRS, 34095 Montpellier, France.

${ }^{3}$ Sorbonne Université, Institut des Matériaux de Paris Centre (IMPC), 4 place Jussieu, 75252 Paris cedex 05, France.

4 Sorbonne Université, Collège de France, Laboratoire de Chimie de la Matière Condensée de Paris (LCMCP), 4 place Jussieu, 75252 Paris cedex 05, France.

${ }^{5}$ Institut Universitaire de France (IUF), MESRI, 1 rue Descartes, 75231 Paris cedex 05, France.

${ }^{6}$ Department of Applied Physics, The Hong Kong Polytechnic University, Hong Kong, China.

*Correspondence to: damien.voiry@umontpellier.fr; nicolas.onofrio@ polyu.edu.hk.

\section{Supplementary Materials}




\section{Table of content}

Sections $\quad$ Page

Enhanced sieving from exfoliated $\mathrm{MoS}_{2}$ membranes via covalent functionalization ........ 1

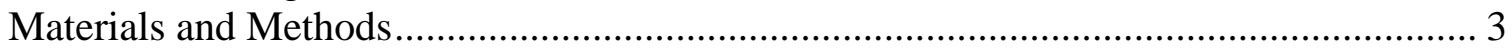

Size distribution of the single-layer $\mathrm{MoS}_{2}$ nanosheets ................................................... 3

Zeta potential measurements....................................................................................... 4

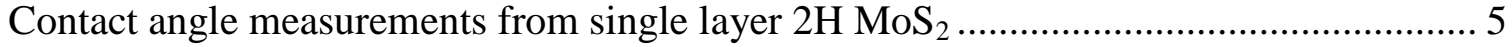

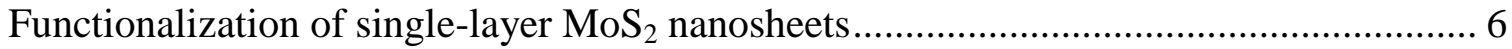

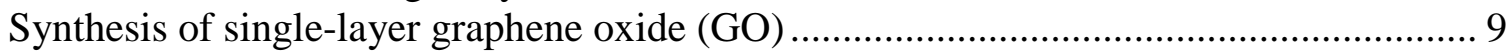

Physical characterizations ....................................................................................... 10

ATR-FTIR spectroscopy measurements on functionalized $\mathrm{MoS}_{2}$ membranes.................. 11

TGA measurements on functionalized $\mathrm{MoS}_{2}$ membranes ............................................. 12

${ }^{13} \mathrm{C}$ Nuclear Magnetic resonance spectroscopy (NMR) ................................................. 14

X-ray photoelectron spectroscopy (XPS) on functionalized $\mathrm{MoS}_{2}$ membranes ............... 15

Raman spectroscopy on functionalized $\mathrm{MoS}_{2}$ nanosheets.............................................. 19

$\mathrm{X}$-Ray diffraction (XRD) on $\mathrm{MoS}_{2}$ membranes ………............................................ 21

Stability of the functionalized $\mathrm{MoS}_{2}$ membranes ………………………………….... 23

Controlled of the swelling in the functionalized $\mathrm{MoS}_{2}$-based membranes ....................... 24

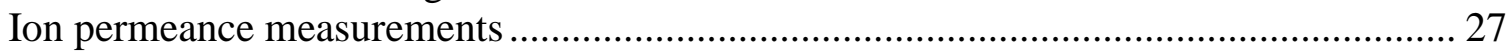

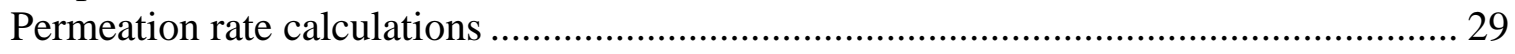

Binding energy between the ions and the functional groups ............................................ 30

Forward osmosis (FO)-driven measurements ............................................................. 33

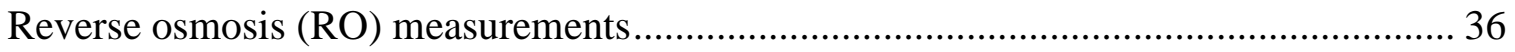

Stability of the water flux in dead-end configuration ....................................................... 39

Influence of the membrane thickness on the functionalization degree and stacking order

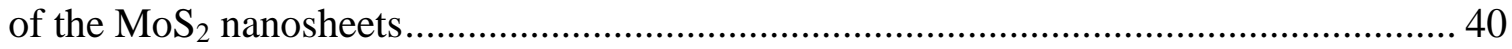

Velocity profile and slip length measurements............................................................... 46

Molecular sieving performances under reversed osmosis conditions............................... 47

Desalination performance under reverse osmosis conditions ........................................... 49

Comparison of the performance of the $\mathrm{MoS}_{2}$ membranes with the state of the arts ........ 51

Molecular dynamics simulations ................................................................................. 57

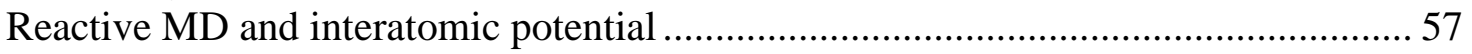

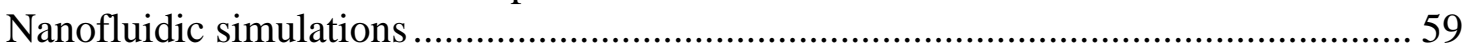

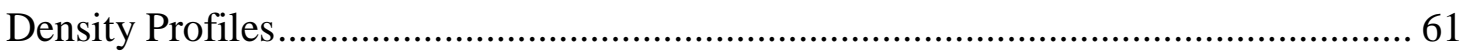

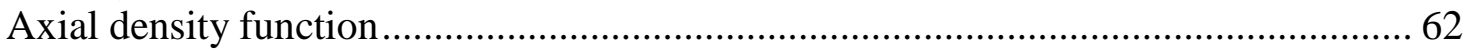

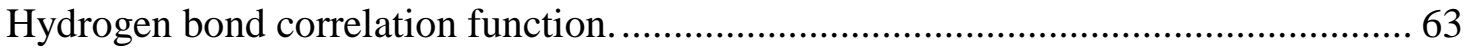

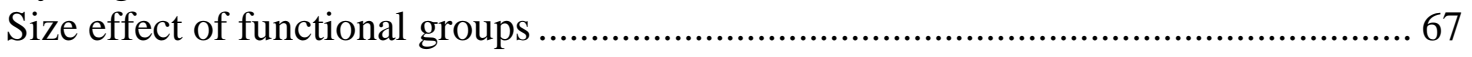

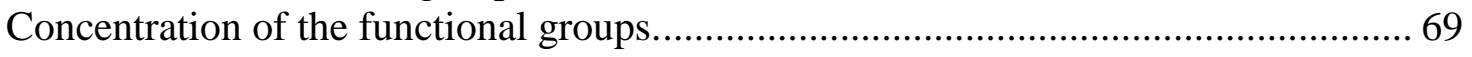

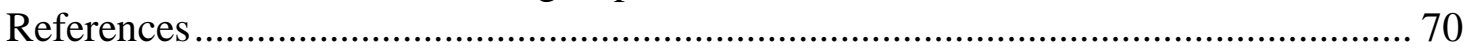




\section{Materials and Methods}

Bulk $\mathrm{MoS}_{2}$ powder, iodomethane, 2-iodoacetamide, 2-iodoethanol (> $\left.99 \%\right)$, acetamide, n-butyllithium (1.6 $\mathrm{M}$ in hexane) and Nickel(II) phthalocyanine-tetrasulfonic acid tetrasodium salt were purchased from Sigma Aldrich. Organic solvents were ordered from either Sigma Aldrich or Alfa Aesar and used as received.

\section{Size distribution of the single-layer $\mathrm{MoS}_{2}$ nanosheets}

The size of the single-layer nanosheets was analyzed by atomic force microscopy (Digital Instruments Nanoscope IV in tapping mode with standard cantilevers with spring constant of $40 \mathrm{~N} / \mathrm{m}$ and tip curvature $<10 \mathrm{~nm}$ ). Supplementary Figure 1 shows the statistics of the size distribution of the exfoliated $\mathrm{MoS}_{2}$ nanosheets. The average length and width are estimated to 340 and $230 \mathrm{~nm}$ respectively.
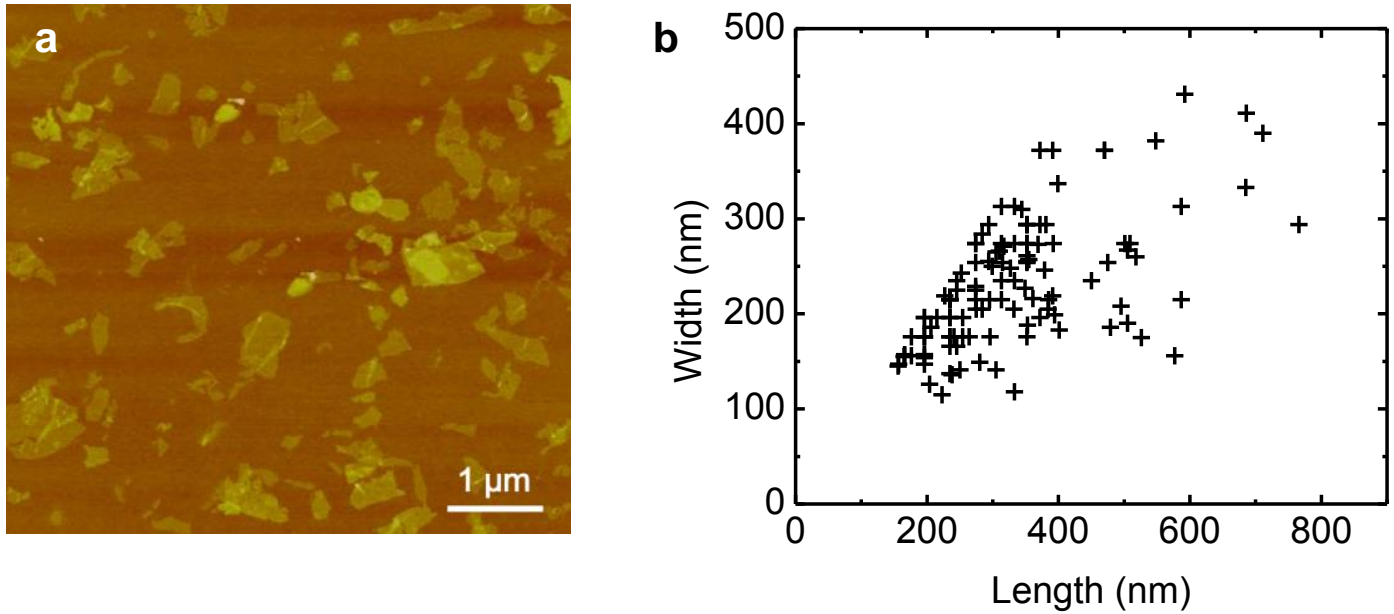

Supplementary Figure 1. (a) AFM image of chemically exfoliated $\mathrm{MoS}_{2}$ nanosheets. (b) Size distribution of the exfoliated $\mathrm{MoS}_{2}$ nanosheets measured by Atomic Force Microscopy. 


\section{Zeta potential measurements}

The Zeta potential measurements ( $\zeta$ ) of as-exfoliated and functionalized (1T) $\mathrm{MoS}_{2}$ nanosheets were performed using an Anton Paar Litesizer 500 Particle Analyzer at neutral $\mathrm{pH}$ (in water). Our results shows that the zeta potential for pristine $\mathrm{MoS}_{2}$ nanosheets is $-45.4 \pm 0.8 \mathrm{mV}$ confirming the presence of a large excess of negative charges in the exfoliated materials. This result agrees with previous results from the literature ${ }^{1}$. As expected, the Zeta potential decreases after covalent functionalization because of the consumption of the extra electrons during the functionalization reaction. We found a remarkable agreement between the contact angle and the Zeta potential for the different $\mathrm{MoS}_{2}$ membranes. More importantly the evolution of the Zeta potential closely follows the trend of polarity of the functional groups: $\zeta_{\mathrm{MoS}_{2}-\text { Acet. }}>\zeta_{\mathrm{MoS}_{2}-\mathrm{EtOH}}>\zeta_{\mathrm{MoS}_{2}-\mathrm{Met}}$ (the values are presented in Table 3 below). The Zeta potential measurements suggest that the nanosheets remain partially charged after functionalization and that the density of charges can be tuned by the nature of the functional groups grafted on the nanosheets.

Supplementary Table 1 Zeta potential and contact angle measurements of as-exfoliated (1T) $\mathrm{MoS}_{2}$ and functionalized $\mathrm{MoS}_{2}$

\begin{tabular}{|c|c|c|}
\hline Samples & Zeta potential $(\boldsymbol{\xi}, \mathbf{m V})$ & Contact angle \\
\hline $1 \mathrm{~T} \mathrm{MoS}$ & & $58^{\circ}$ \\
\hline $2 \mathrm{H} \mathrm{MoS}$ & $-45.4 \pm 0.8$ & $98^{\circ}$ \\
\hline $\mathrm{MoS}_{2}$-Acet. & $-30($ Ref 6) & $72^{\circ}$ \\
\hline $\mathrm{MoS}_{2}$-Met. & $-39.3 \pm 0.4$ & $90^{\circ}$ \\
\hline $\mathrm{MoS}_{2}$-EtOH & $-29.6 \pm 0.3$ & $80^{\circ}$ \\
\hline
\end{tabular}




\section{Contact angle measurements from single layer $2 \mathrm{H} \mathbf{M o S}_{2}$}

The contact angle of the as-exfoliated (1T) $\mathrm{MoS}_{2}$ membranes is $58^{\circ}$. We compared this value with the contact angle of single-layer $2 \mathrm{H} \mathrm{MoS}$ grown by chemical vapor deposition ${ }^{2}$. We found that the contact angle of $2 \mathrm{H} \mathrm{MoS}_{2}$ is $\sim 95^{\circ}$ (Supplementary Figure 2). This value is close to the ones reported by Chow et al. for single-layer $\mathrm{MoS}_{2}$ and $\mathrm{WS}_{2}$ : $82^{\circ}$ and $85^{\circ}$, respectively. The lower contact angle of as-exfoliated $\mathrm{MoS}_{2}$ membranes is attributed to the presence of charge in chemically exfoliated $1 \mathrm{~T} \mathrm{MoS}_{2}$. Indeed Heising et al. have reported that there is a $30 \%$ excess of charge in the chemically exfoliated $\mathrm{MoS}_{2}$ nanosheets ${ }^{4}$. This is also confirmed by the Zeta potential of as-exfoliated $\mathrm{MoS}_{2}$ which is significantly larger than that of functionalized $\mathrm{MoS}_{2}$ and $2 \mathrm{H} \mathrm{MoS}_{2}{ }^{5}:-45.4 \mathrm{mV}$ vs. -30 $\mathrm{mV}$ for $1 \mathrm{~T}$ and $2 \mathrm{H} \mathrm{MoS}$, respectively.

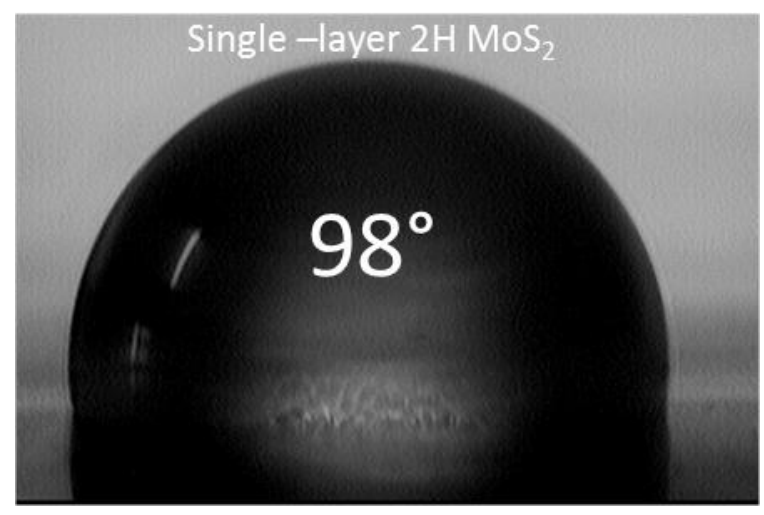

Supplementary Figure 2. Water contact angle for single-layer $\mathrm{MoS}_{2}$ grown by CVD and deposited on $\mathrm{SiO}_{2} / \mathrm{Si}$ wafer. 


\section{Functionalization of single-layer $\mathrm{MoS}_{2}$ nanosheets}

Single-layer $\mathrm{MoS}_{2}$ nanosheets were functionalized using organohalide reagents. Functionalization of the nanosheets was performed either in solution or directly on the membranes. According to the (002) peak obtained from X-ray diffraction (XRD), the $d$ space is estimated to about $13 \AA\left(2 \theta=6.6^{\circ}\right)$ and $6.18 \AA\left(2 \theta=14.4^{\circ}\right)$ in the case of asfiltered and dried $\mathrm{MoS}_{2}$ membrane respectively (Supplementary Figure 3). The large physical gaps between the $\mathrm{MoS}_{2}$ nanosheets allow the reagents and the solvent to slowly diffuse between the layers. The reagent solution was let to slowly diffuse through the $\mathrm{MoS}_{2}$ membrane for 48 hours after what the membrane was rinsed with 2-propanol $(4 \times 50$ $\mathrm{mL})$, ethanol $(4 \times 50 \mathrm{~mL})$ and water $(4 \times 50 \mathrm{~mL})$. The organohalide reagent is typically dissolved in the 2:1 water:2-propanol solution at the concentration of $15 \mathrm{mM}$. For example, for a membrane of $250 \mathrm{~nm}$ in thickness, the typical mass of $\mathrm{MoS}_{2}$ nanosheets is estimated to be $1.14 \mathrm{mg}\left(7.1 \times 10^{-6} \mathrm{~mol}\right)$. A 10 -fold excess of iodomethane corresponding to $7.1 \times 10^{-5} \mathrm{~mol}(0.010 \mathrm{~g})$ was used for the functionalization and dissolved in $4.7 \mathrm{~mL}$ of water:2-propanol solution. The excess of reagents and sub-products of the reaction was eliminated by rising with 2-propanol $(4 \times 50 \mathrm{~mL})$, ethanol $(4 \times 50 \mathrm{~mL})$ and water $(4 \times 50$ $\mathrm{mL}$ ). Finally the membranes were dried in air at $60^{\circ} \mathrm{C}$ under atmospheric pressure. Cross section SEM observations confirm the stacking of the nanosheets in the different functionalized $\mathrm{MoS}_{2}$ membranes while the presence of aggregates and crumpling is not detected (Supplementary Figure 5). 


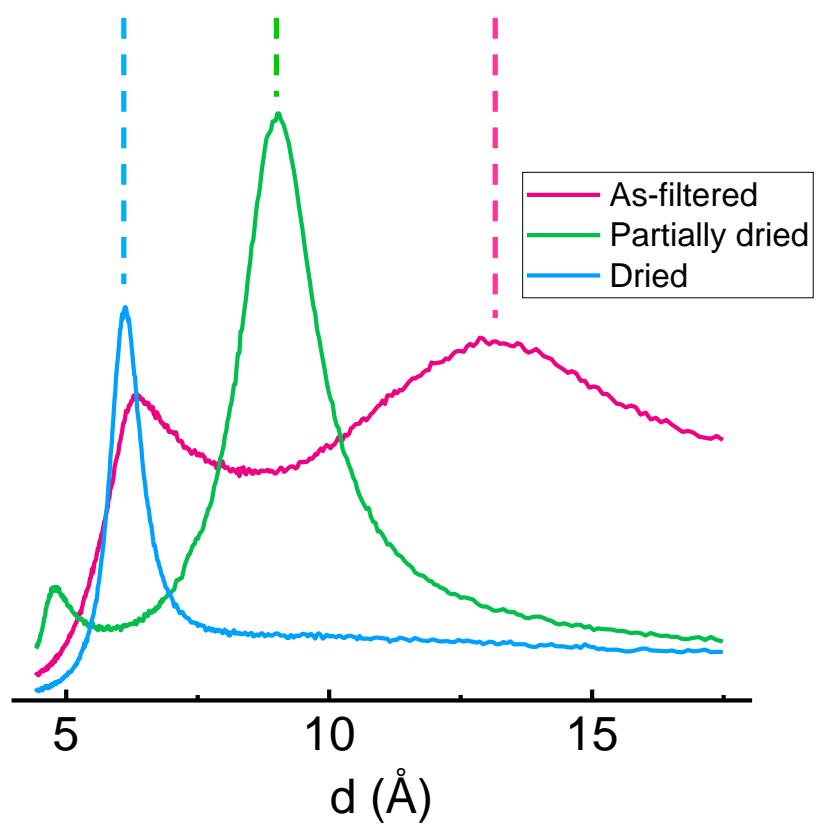

Supplementary Figure 3. X-ray diffraction spectra centered around the (002) peak (dashed line) from as-filtered (pink), partially dried (green) and dried (blue) nanolaminate $\mathrm{MoS}_{2}$ membrane

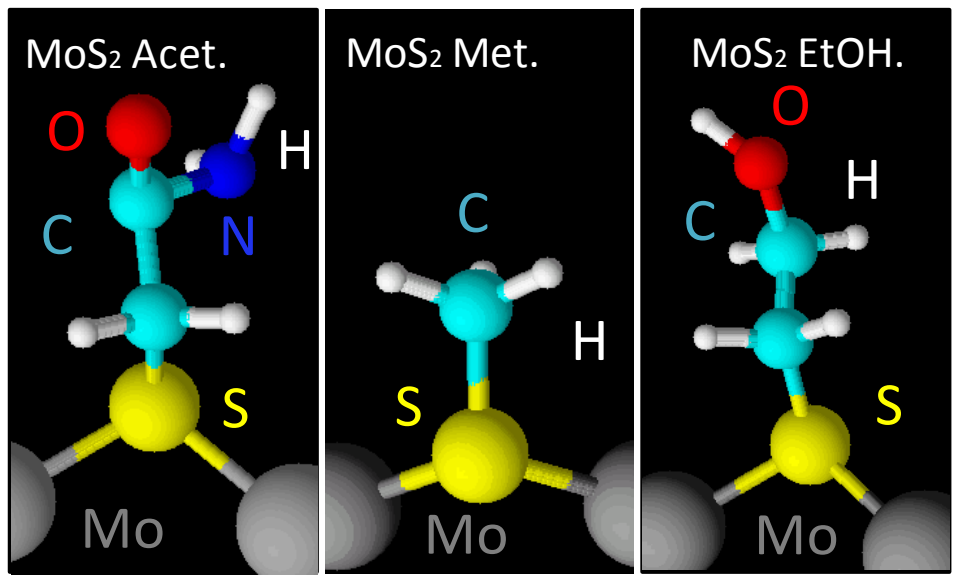

Supplementary Figure 4. Schematic representation of the functional groups on the surface of the $\mathrm{MoS}_{2}$ nanosheets. 

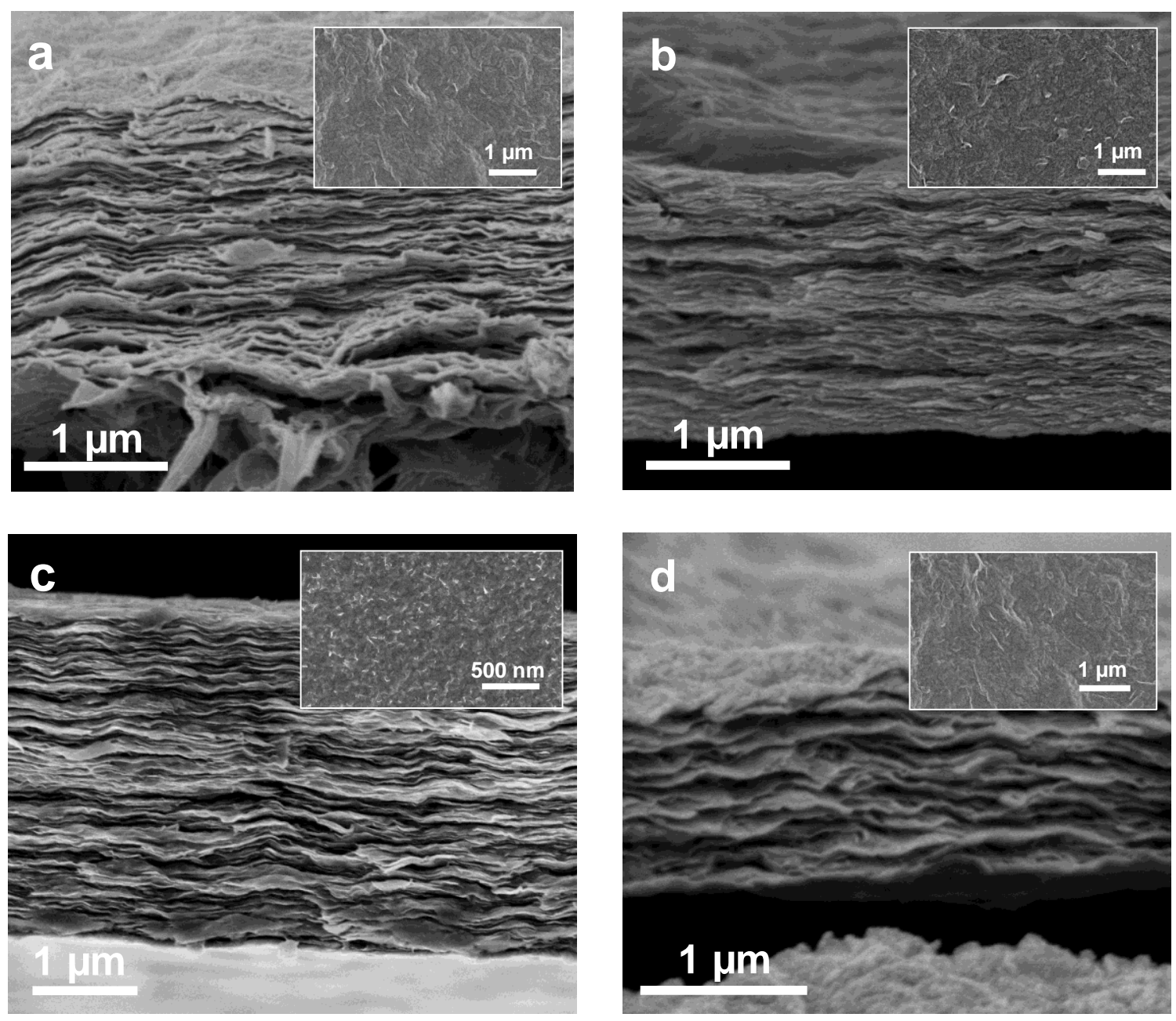

Supplementary Figure 5. Cross section images of pristine (a), acetamide (b), methyl (c) and ethyl-2-ol (d) functionalized $\mathrm{MoS}_{2}$ membranes. Insets: Top view observations of the corresponding membranes under SEM.

To further attest the covalent bonding of functional groups on $\mathrm{MoS}_{2}$, we let the exfoliated $\mathrm{MoS}_{2}$ nanosheets reacting with acetamide following the same methodology. Supplementary Figure 6 shows the X-ray diffraction signals from membranes made of exfoliated $\mathrm{MoS}_{2}$ nanosheets treated with acetamide and 2-iodoacetamide. We observe no shift of the (002) peak in comparison to non-functionalized $\mathrm{MoS}_{2}$ membranes. This indicates that the washing step, after functionalization, efficiently removes any 
intercalated species non-covalently bonded to the nanosheets. It also demonstrates the key role played by the iodine groups in the functionalization reaction.

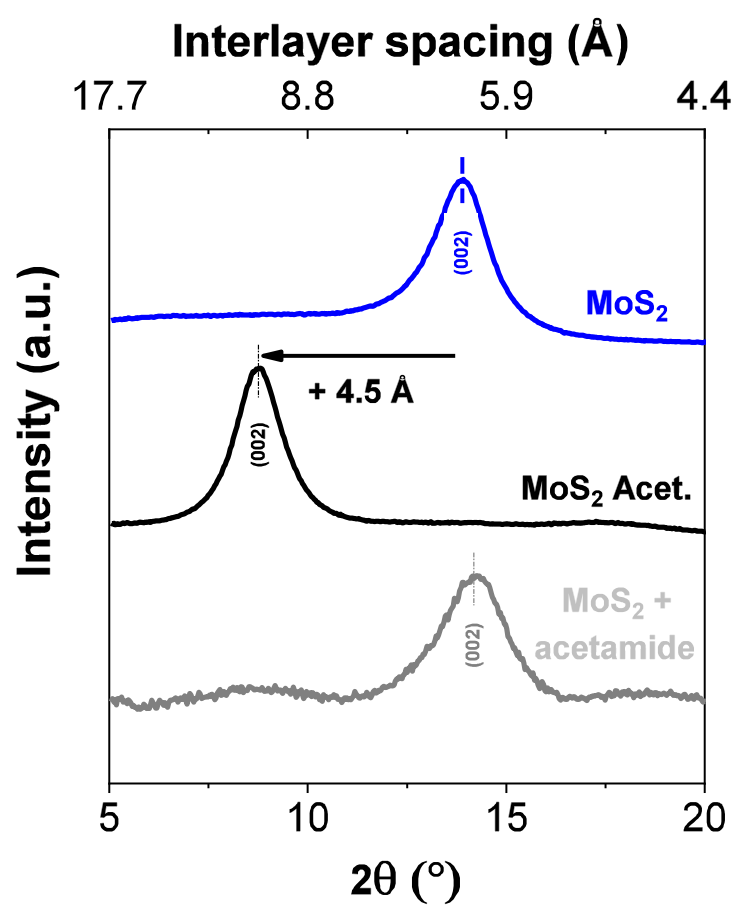

Supplementary Figure 6. X-Ray diffraction spectra from non-functionalized $\mathrm{MoS}_{2}$ membranes compared to $\mathrm{MoS}_{2}$ membranes treated with acetamide and 2-iodoacetamide.

\section{Synthesis of single-layer graphene oxide (GO)}

GO was prepared from natural graphite by the modified Hummers' method. ${ }^{6}$ Graphite powder $(1.5 \mathrm{~g})$ was mixed with $\mathrm{H}_{2} \mathrm{SO}_{4}(50.7 \mathrm{~mL})$ and sodium nitrate $(1.14 \mathrm{~g})$. In an ice bath, $\mathrm{KMnO}_{4}(6 \mathrm{~g})$ was added slowly while stirring. After 72 hours, $150 \mathrm{~mL}$ solution of $\mathrm{H}_{2} \mathrm{SO}_{4}$ was slowly added and the reaction was finally quenched by the addition of $4.5 \mathrm{~mL}$ of $\mathrm{H}_{2} \mathrm{O}_{2}(30 \%)$. Metal ion impurities were removed by treating the solution with $10 \%$ $\mathrm{HCl}$. The solution was then washed using dialysis (molecular weight cut-off $=14,000$, 
Sigma-Aldrich) to completely remove metal ions and acids. The GO nanosheets were centrifuged at 3,000 rpm for 3 minutes to remove the non-exfoliated particles.

\section{Physical characterizations}

X-ray photoelectron spectroscopy (XPS, Thermo Scientific K-Alpha spectrometer) was performed using a $\mathrm{Al}-\mathrm{K} \alpha$ microfocused monochromatized source $(1486.7 \mathrm{eV})$ with a resolution of $0.6 \mathrm{eV}$ and a spot size of $400 \mu \mathrm{m}$. High resolution transmission electron microscopy images (HRTEM) were obtained using HRTEM JEOL 2200 FS microscope with an emission gun operating at $200 \mathrm{kV}$. Elemental mappings have been performed using Scanning Transmission Electron Microscope (STEM) coupled with energy dispersive X-ray spectroscopy (EDS) using SDD JEOL (30 $\left.\mathrm{mm}^{2}-129 \mathrm{eV}\right)$. Functionalized $\mathrm{MoS}_{2}$ nanosheets were characterized using RAMAN spectroscopy (Renishaw 1000 system operating at $514 \mathrm{~nm}$ ), attenuated total reflectance FTIR (Nicolet Nexus FT-IR spectrometer equipped with a ATR Diamant Golden Gate) and solid state

${ }^{13} \mathrm{C}$ CP MAS NMR (Bruker Avance $700 \mathrm{MHz}$ ). Organohalide reagents were analyzed by ${ }^{13} \mathrm{C}$ solution nuclear magnetic resonance (Avance $300 \mathrm{MHz}$ instrument from Bruker); both 2-iodoethanol and iodomethane were analyzed using $\mathrm{CDCl}_{3}(77.3 \mathrm{ppm})$ as an internal solvent whereas 2-iodoactetamide spectra was recorded in $\mathrm{D}_{2} \mathrm{O}$. X-ray Diffraction was performed on an Xpert Pro apparatus (Philips Pana Analytical, $\mathrm{Cu} \mathrm{K} \alpha ; \lambda$ $=1.5418 \AA$ ) operating at $40 \mathrm{kV}$ and $20 \mathrm{~mA}$. Scanning electron microscopy (SEM) was carried out on a Zeiss Sigma Field Emission SEM with an Oxford INCA PentaFETx3 EDS system (model 8100). 


\section{ATR-FTIR spectroscopy measurements on functionalized $\mathrm{MoS}_{2}$ membranes}

ATR-FTIR spectroscopy was performed on $\mathrm{MoS}_{2}$-derived materials (Supplementary Figure 7). The ATR-FTIR spectra of functionalized $\mathrm{MoS}_{2}$ nanosheets bring the first evidence of covalent grafting of the functional groups. The S-C stretching is identified at $\sim 710 \mathrm{~cm}^{-1}$ for both acetamide-, methyl- and ethyl-2-ol- functionalized $\mathrm{MoS}_{2}$, whereas the stretching mode $\gamma_{\mathrm{W}}$ for $\mathrm{S}_{-} \mathrm{CH}_{2}$ - appears at $1151 \mathrm{~cm}^{-1}$ and $1129 \mathrm{~cm}^{-1}$ for acetamide and ethyl-2-ol- functionalized $\mathrm{MoS}_{2}$. The deformation modes $\delta$ for C-H are clearly visible at $1293 \mathrm{~cm}^{-1}$ and $954 \mathrm{~cm}^{-1}$ in methyl- functionalized $\mathrm{MoS}_{2}{ }^{7}$.

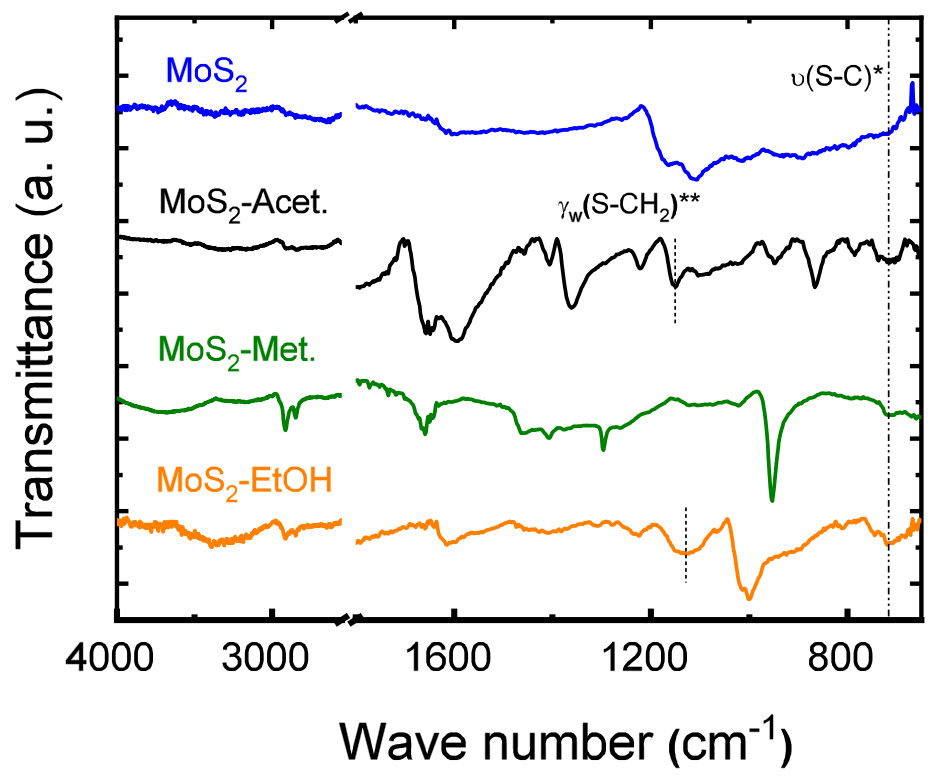

Supplementary Figure 7. ATR-FTIR spectra of pristine (non-functionalized) $\mathrm{MoS}_{2}$ (blue), acetamide- functionalized $\mathrm{MoS}_{2}$ (black), methyl- functionalized $\mathrm{MoS}_{2}$ (green) and ethyl-2-ol-functionalized $\mathrm{MoS}_{2}$ (orange); $\left(*:{ }^{7} ; * *{ }^{8}\right)$.

Acetamide- functionalized $\mathrm{MoS}_{2}$ nanosheets have been further studied on Supplementary Figure 8 wherein ATR-FTIR spectra of pristine $\mathrm{MoS}_{2}$ (blue trace) is compared to 
acetamide- functionalized $\mathrm{MoS}_{2}$ (black), 2-iodoacetamide (brown), and pure acetamide compound (red). Acetamide functionalized $\mathrm{MoS}_{2}$ displays strong signals at $1655 \mathrm{~cm}^{-1}$ and $1605 \mathrm{~cm}^{-1}$ attributed to $\mathrm{C}=\mathrm{O}$ and $\mathrm{N}-\mathrm{H}$ stretching ${ }^{7}$. Remarkably the FTIR response for acetamide- functionalized $\mathrm{MoS}_{2}$ appears very similar to acetamide.

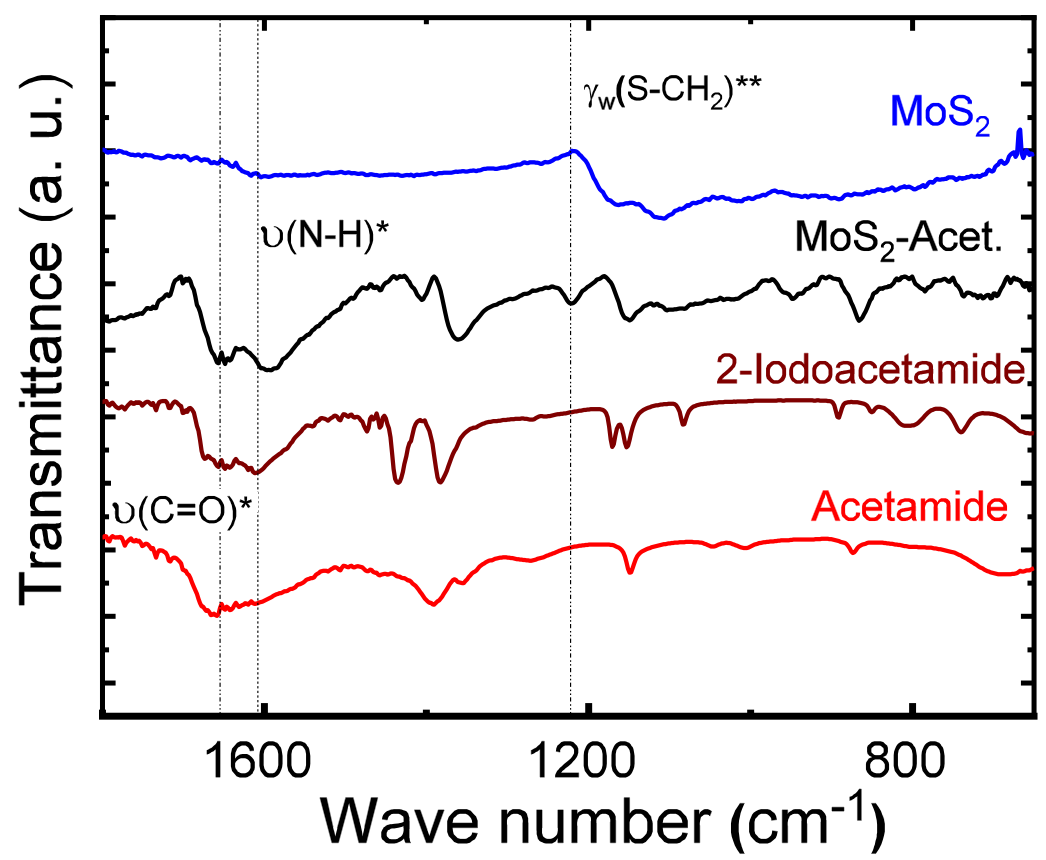

Supplementary Figure 8. ATR-FTIR spectra of pristine (non-functionalized) $\mathrm{MoS}_{2}$ compared with covalently functionalized $\mathrm{MoS}_{2}$ nanosheets with acetamide groups (black trace), 2-iodoacetamide (brown trace) and acetamide (red trace); $\left(*:{ }^{7} ; * * 8^{8}\right)$.

\section{TGA measurements on functionalized $\mathrm{MoS}_{2}$ membranes}

Thermogravimetric analysis (TGA) were performed on a TGA Q500 Thermogravimetric Analyzer equipped with Thermal Analysis Controller. After purging the system for 30 min at $50^{\circ} \mathrm{C}$, samples were heated under nitrogen at $10^{\circ} \mathrm{C}-\min ^{-1}$ from $50^{\circ} \mathrm{C}$ up to $800{ }^{\circ} \mathrm{C}$. The weight loss has been recorded for pristine $\mathrm{MoS}_{2}$ and functionalized $\mathrm{MoS}_{2}$ nanosheets 
(Supplementary Figure 9a). The derivative signal from pristine $\mathrm{MoS}_{2}$ nanosheets remains relatively flat. At the opposite, the derivative of the weight loss shows peaks at $223^{\circ} \mathrm{C}$, $248^{\circ}$ and $378^{\circ} \mathrm{C}$ for the degradation of ethy-2-ol, acetamide and methyl groups respectively (Supplementary Figure 9b). The extent of functionalization was calculated from the weight loss at $500^{\circ} \mathrm{C}$ and assuming that the difference between the weight loss from pristine and functionalized $\mathrm{MoS}_{2}$ nanosheets is solely coming from the degradation of the grafted functions. The functionalization degrees per sulfur reach $\sim 19$ at.\%, 22 at.\% and $\sim 55$ at. $\%$ for $\mathrm{MoS}_{2}$-Acet., $\mathrm{MoS}_{2}-\mathrm{EtOH}$ and $\mathrm{MoS}_{2}$-Met. respectively. These values are in relatively good agreement with the XPS results obtained by deconvoluting the S-C signals from the S2p regions. We note that the higher functionalization degree for $\mathrm{MoS}_{2}$-Met. is explained by the difficulty in precisely measuring the weight solely coming from the methyl groups.
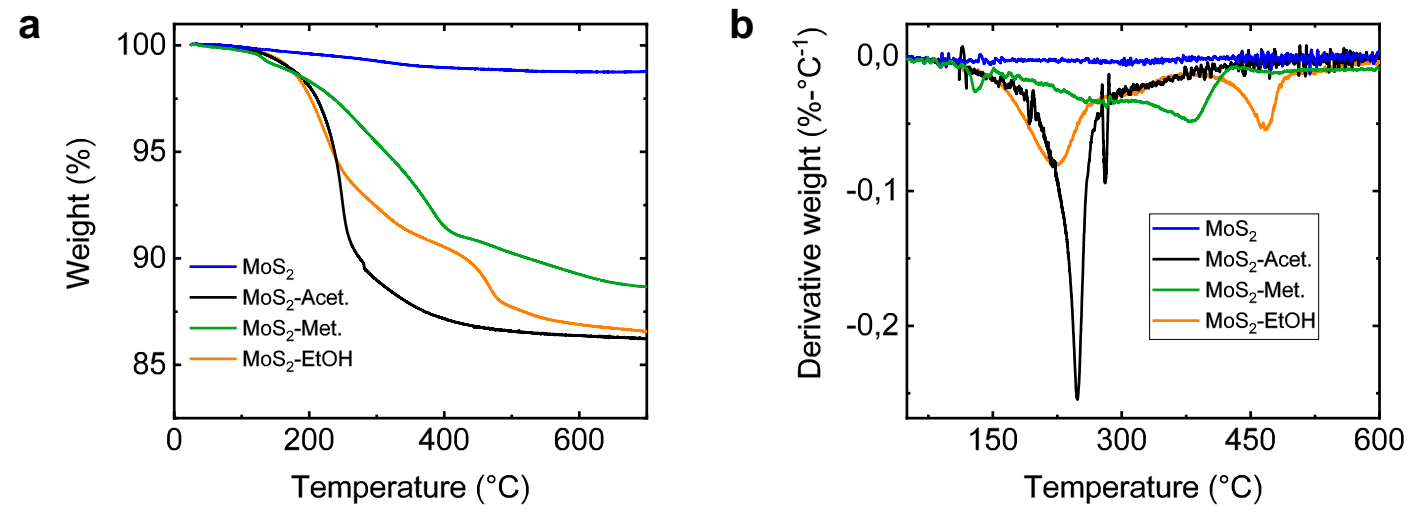

Supplementary Figure 9. (a) Thermogravimetric traces of pristine and functionalized $\mathrm{MoS}_{2}$ nanosheets up to $700^{\circ} \mathrm{C}$ under $\mathrm{N}_{2}$. (b) First derivative of the TGA traces. 


\section{${ }^{13}$ C Nuclear Magnetic resonance spectroscopy (NMR)}

The solid-state ${ }^{13} \mathrm{C}$ CP MAS NMR experiments were performed on a Bruker $700 \mathrm{MHz}$ (16.4 T) spectrometer at a frequency of $176.05 \mathrm{MHz}$, using a $3.2 \mathrm{~mm}$ Bruker MAS probe (contact time: $2 \mathrm{~ms}$, SPINAL-64 $\left\{{ }^{1} \mathrm{H}\right\}$ decoupling, ramp 90 scheme during the polarization transfer, rotation frequency: $12 \mathrm{kHz}$, recycle delay: $4 \mathrm{~s}$, number of scans: between $42 \mathrm{~K}$ and $58 \mathrm{~K}$ ). The samples were prepared by mixing $\mathrm{NaCl}$ with the powders followed by careful packing of the mixture into a $3.2 \mathrm{~mm}$ zirconia rotor. ${ }^{13} \mathrm{C}$ chemical shifts were referenced to tetramethylsilane, using adamantane as a secondary reference.

To confirm the shift of the $\alpha-\mathrm{C}$ after covalent attachment on the $\mathrm{S}$ atoms of the $\mathrm{MoS}_{2}$ nanosheets, liquid-state ${ }^{13} \mathrm{C}$ NMR were performed on the organohalide reagents used for each functionalization route (Supplementary Figure 10). The chemical shift from $\mathrm{O}=C$ $\mathrm{NH}_{2}$ bond in 2-iodoacetamide, and $\mathrm{HO}-\mathrm{CH}_{2}-\mathrm{CH}_{2}$ bond in 2-iodoethanol appear respectively at $174.8 \mathrm{ppm}$ and $63.37 \mathrm{ppm}$. These peaks are not significantly shifted after functionalization (main Figure 2a). The chemical shift of the $\alpha$-C from C-I appears at $2.51 \mathrm{ppm},-23.20 \mathrm{ppm}$ and $8.97 \mathrm{ppm}$ for 2-iodoacetamide, iodomethane and 2iodoethanol, respectively (Supplementary Figure 10). These peaks are largely shifted by 40-50 ppm after covalent grafting on the surface of the $\mathrm{MoS}_{2}$ nanosheets (main Figure 2a). 


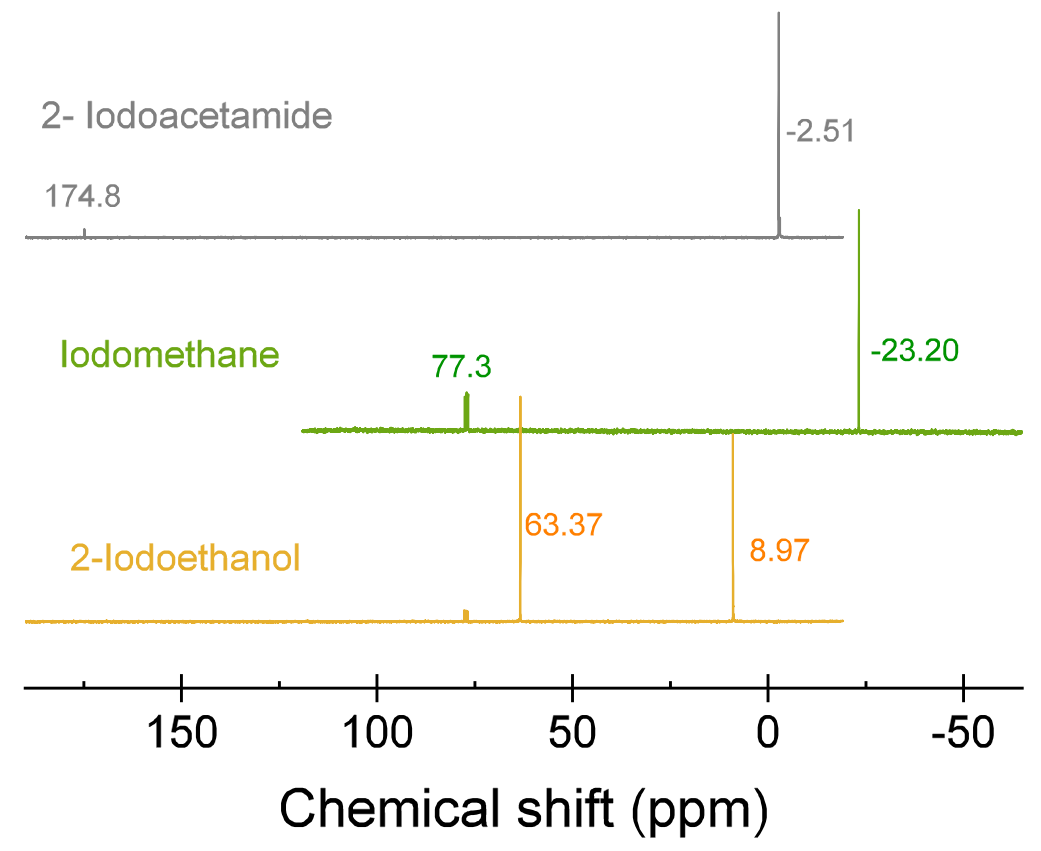

Supplementary Figure 10. Liquid-state ${ }^{13} \mathrm{C}$ NMR spectra of 2-iodoacetamide (grey), iodomethane (green), 2-iodoethanol (orange). The chemical shift observed at $77.3 \mathrm{ppm}$ for 2-iodoethanol and iodomethane originates from $\mathrm{CDCl}_{3}$ used as solvent.

\section{X-ray photoelectron spectroscopy (XPS) on functionalized $\mathrm{MoS}_{2}$ membranes}

Covalent functionalization was also probed by X-ray photoelectron spectroscopy. First from the survey spectra, no iodine was detected, which confirms the successful rinsing of the $\mathrm{MoS}_{2}$ nanosheets after the functionalization reaction (Supplementary Figure 11, left). 


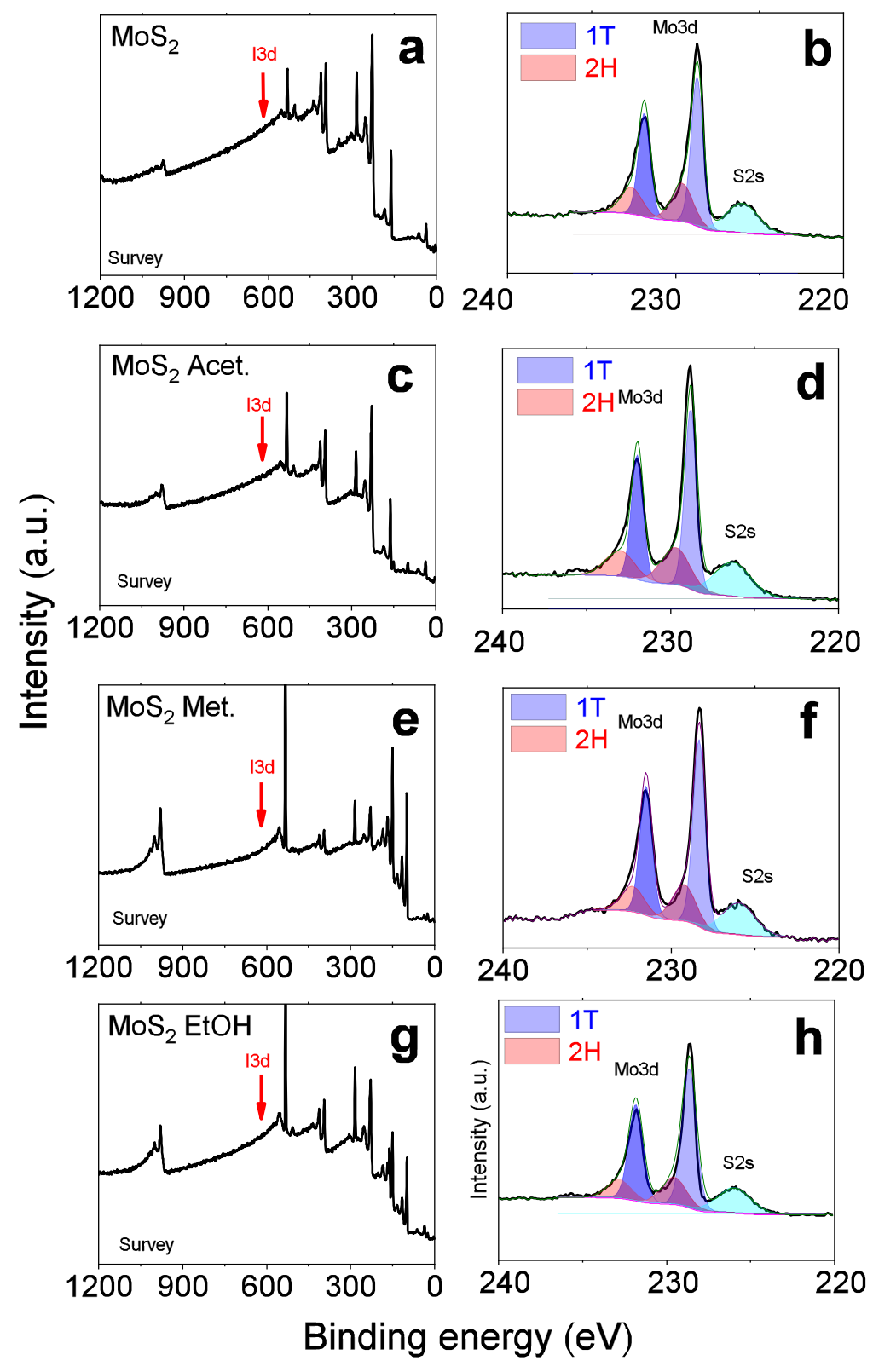

Supplementary Figure 11. XPS survey (left) and Mo3d (right) spectra from the pristine and functionalized $\mathrm{MoS}_{2}$ nanosheets.

X-ray photoelectron spectra for the $\mathrm{S} 2 \mathrm{p}$ and Mo3d regions are shown in Supplementary Figure 12. As reported previously in the case of chemically exfoliated $\mathrm{MoS}_{2}$, the S2p and Mo3d regions can be convoluted with components from the $1 \mathrm{~T}$ and $2 \mathrm{H}$ phases. The presence of functional groups can be seen on the $\mathrm{S} 2 \mathrm{p}$ regions (158-166 
eV) whereas signals from the Mo3d regions remain virtually unchanged (389-400 eV). Such changes are attributed to the formation of the S-C bonds (in green) due to the covalent grafting on the $\mathrm{S}$ atoms.

Another evidence of functionalization is brought from the N1s region in the case of the acetamide-functionalized $\mathrm{MoS}_{2}$ (Supplementary Figure 12). For comparison, no N1s can be detected at $\sim 400 \mathrm{eV}$ from the pristine non-functionalized $\mathrm{MoS}_{2}$ nanosheets. The fitting of the N1s signals enables quantification of the functional groups. The N/Mo is estimated to 40 at.\% giving a functionalization degree of 20 at.\% per sulfur atoms. Similar functionalization degree has been measured from S-C signals obtained by the deconvolution of the S2p signals (Supplementary Table 2). 


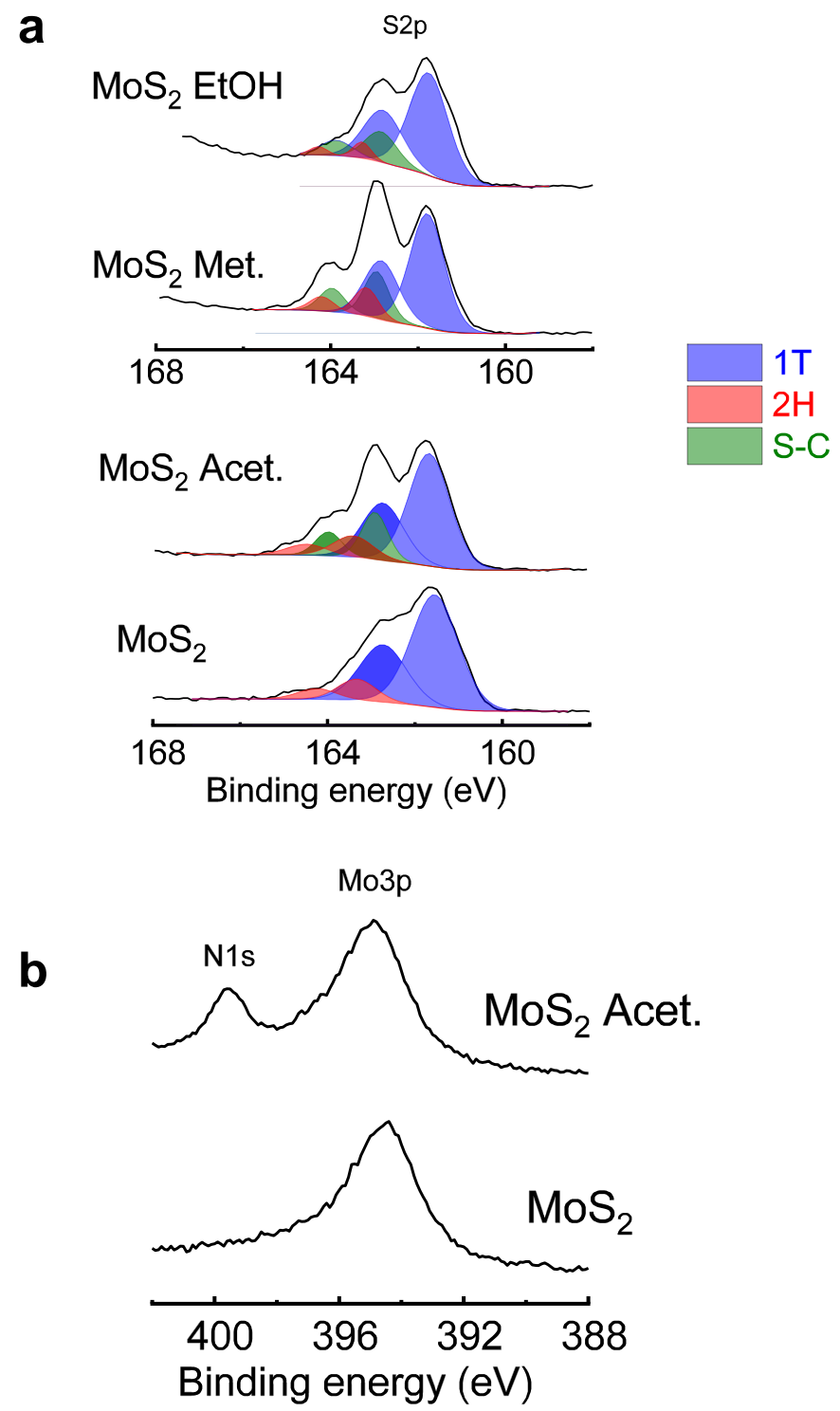

Supplementary Figure 12. X-ray photoelectron spectra of S2p (a) and N1s (b) regions from the pristine and functionalized $\mathrm{MoS}_{2}$ nanosheets. The functionalization degree refers to the percentage of functionalization group per $\mathrm{S}$ atoms.

Supplementary Table 2. Details of the XPS results for $\mathrm{MoS}_{2}$ and functionalized $\mathrm{MoS}_{2}$ samples. The functionalization degree refers to the percentage of functionalization group per S atoms. 


\begin{tabular}{|c|c|c|c|c|c|}
\hline \multicolumn{6}{|c|}{$\mathbf{M o S}_{2}$-Met. } \\
\hline & \multirow{2}{*}{$\begin{array}{c}\text { Peak } \\
\text { position } \\
(\mathrm{eV})\end{array}$} & \multirow{2}{*}{$\begin{array}{c}\text { FWHM } \\
(\mathrm{eV})\end{array}$} & \multirow[b]{2}{*}{ Area (P) CPS } & \multirow[b]{2}{*}{ at. $\%$} & \multirow{2}{*}{$\begin{array}{c}\text { Funct. degree } \\
(\%)\end{array}$} \\
\hline & & & & & \\
\hline \multirow{2}{*}{$1 T$} & 161.8 & \multirow{2}{*}{0.93} & 7817.9 & \multirow{2}{*}{62.5} & \\
\hline & 162.9 & & 3993.5 & & \\
\hline \multirow{2}{*}{$2 \mathrm{H}$} & 163.2 & \multirow{2}{*}{0.67} & 1595.1 & \multirow{2}{*}{12.8} & \\
\hline & 164.2 & & 814.8 & & \\
\hline \multirow{2}{*}{ S-C } & 162.9 & \multirow{2}{*}{0.88} & 3091.5 & \multirow{2}{*}{24.7} & \multirow{2}{*}{25} \\
\hline & 164.0 & & 1579.17 & & \\
\hline \multicolumn{6}{|c|}{$\mathrm{MoS}_{2}$-Acet. } \\
\hline \multirow{2}{*}{$1 T$} & 161.7 & \multirow{2}{*}{1.11} & 6375.6 & \multirow{2}{*}{68.3} & \\
\hline & 162.7 & & 3256.7 & & \\
\hline \multirow{2}{*}{$2 \mathrm{H}$} & 163.4 & \multirow{2}{*}{1.08} & 1186.3 & \multirow{2}{*}{12.7} & \\
\hline & 164.5 & & 605.9 & & \\
\hline \multirow{2}{*}{ S-C } & 162.9 & \multirow{2}{*}{0.70} & 1767.2 & \multirow{2}{*}{19} & \multirow{2}{*}{19} \\
\hline & 164.0 & & 899.8 & & \\
\hline \multicolumn{6}{|c|}{$\mathrm{MoS}_{2}$-EthOH } \\
\hline \multirow{2}{*}{$1 \mathrm{~T}$} & 161.65 & \multirow{2}{*}{1.05} & 5715.3 & \multirow{2}{*}{75.8} & \\
\hline & 162.7 & & 2919.4 & & \\
\hline \multirow{2}{*}{$2 \mathrm{H}$} & 163.18 & \multirow{2}{*}{0.48} & 416.8 & \multirow{2}{*}{5.5} & \\
\hline & 164.18 & & 212.9 & & \\
\hline \multirow{2}{*}{$S-C$} & 162.75 & 087 & 1406.9 & 187 & 10 \\
\hline & 163.77 & 0.01 & 718.7 & 10.1 & 17 \\
\hline
\end{tabular}

\section{Raman spectroscopy on functionalized $\mathrm{MoS}_{2}$ nanosheets}

1T phase of $\mathrm{MoS}_{2}$ has two Raman modes, the in-plane mode $\mathrm{E}_{2 \mathrm{G}}^{1}$ and the out-of-plane mode $A_{1 G}$, as well as $J_{1}, J_{2}$ and $J_{3}$ vibrational modes recently identified by Calandra ${ }^{9}$. Supplementary Figure 13 shows the Raman spectra of $\mathrm{MoS}_{2}$-derived materials where 
only a weak signal of the $\mathrm{E}_{2 \mathrm{G}}^{1}$ mode is observed for pristine $\mathrm{MoS}_{2}$ (blue trace); indicating a majority of 1T-phase $\mathrm{MoS}_{2}$. In accordance with the literature, $\mathrm{J}_{1}, \mathrm{~J}_{2}$ and $\mathrm{J}_{3}$ vibrational modes are observed at $147 \mathrm{~cm}^{-1}, 236 \mathrm{~cm}^{-1}$, and $332 \mathrm{~cm}^{-1}$. As previously reported by Voiry et al. ${ }^{7}$, the signal from the $1 \mathrm{~T}$ phase is preserved after functionalization. The $\mathrm{A}_{1 \mathrm{G}}$ band splits into two signals due to the covalent binding on the $\mathrm{S}$ atom. Both $\mathrm{J}_{1}$ and $\mathrm{J}_{3}$ widen and get downshifted whereas $J_{2}$ peak gets significantly stronger and upshifted. In addition, strong bands attributed to the anchorage of molecules on the nanosheets can be detected. New peaks appear at $\sim 260 \mathrm{~cm}^{-1}$ and at $\sim 320 \mathrm{~cm}^{-1}$ for all the functionalized samples clearly indicating new vibrational modes due to the covalent attachment on the sulfur atoms. A new peak is also detected at $\sim 210 \mathrm{~cm}^{-1}$ for both acetamide- and methylfunctionalized $\mathrm{MoS}_{2}$.

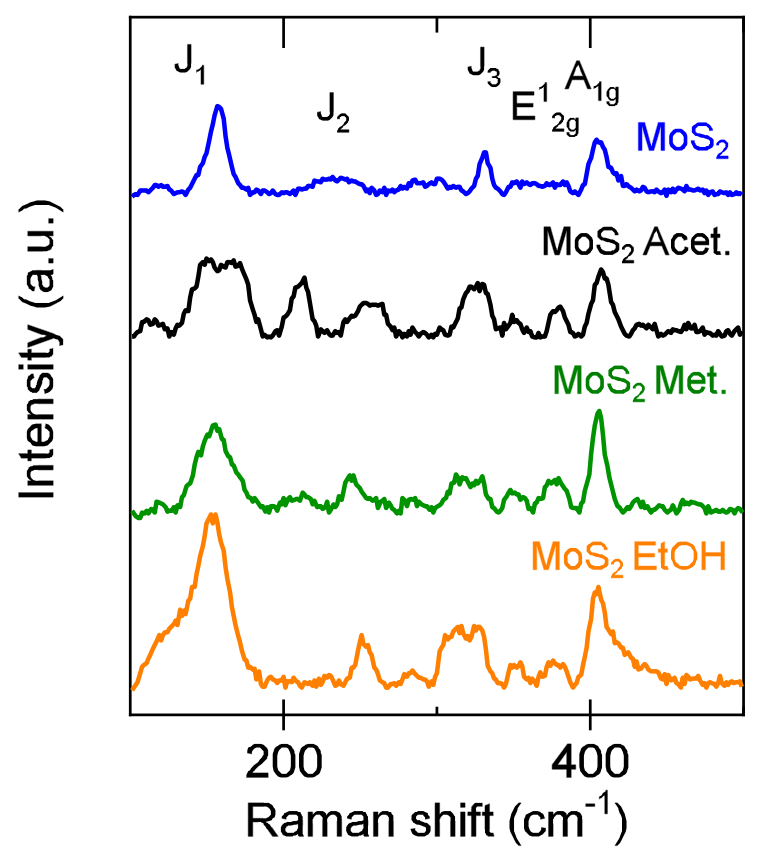

Supplementary Figure 13. Raman spectra of pristine (non-functionalized) $\mathrm{MoS}_{2}$ (blue), acetamide-functionalized $\mathrm{MoS}_{2}$ (black), methyl-functionalized $\mathrm{MoS}_{2}$ (green) and ethyl-2ol-functionalized $\mathrm{MoS}_{2}$ (orange). 


\section{X-Ray diffraction (XRD) on $\mathrm{MoS}_{2}$ membranes}

The d-spacing between the $\mathrm{MoS}_{2}$ layers can be precisely measured using X-ray diffraction (main Figure 2b-d). Upon lithium intercalation, the d-spacing increases from $2 \theta=14.4^{\circ}$ up to $\sim 7.6^{\circ}$ due to the presence of hydrated Li cations (Supplementary Figure 14). After exfoliation and removing Li cations via centrifugation, the d-spacing in the restacked $\mathrm{MoS}_{2}$ reaches $2 \theta=13.96^{\circ}$. Such a minor change in the d-spacing combined to the relative high intensity of the (002) peak suggests a high degree of alignment of the nanosheets in the membranes.

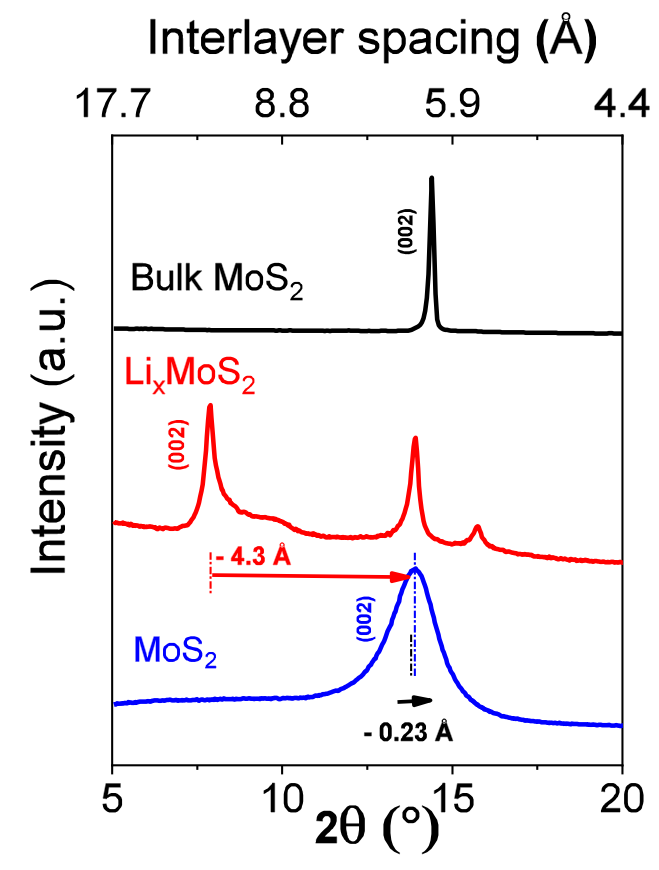

Supplementary Figure 14. X-Ray diffraction spectra from bulk $2 \mathrm{H}-\mathrm{MoS}_{2}$ crystal (black), lithium intercalated $\mathrm{MoS}_{2}$ obtained while liquid exfoliation (red) and pristine non-functionalized $\mathrm{MoS}_{2}$ membrane (blue). 
Interlayer spacing of membranes $d$ are summarized in Supplementary Table 3. and were determined using XRD measurements for all the functionalized $\mathrm{MoS}_{2}$ membranes (see the corresponding diffractograms in main Figure $2 b$ ). The full width at half maximum (FWHMs) of (002) peak are similarly sensitive within all functionalized structures suggesting a comparable degree of functionalization. Interestingly slightly larger $d$-spacing are measured for methyl-functionalized $\mathrm{MoS}_{2}$ membranes. This is surprising as methyl functions are smaller compared to ethyl-2-ol and acetamide (see Supplementary Table 3) suggesting different arrangements of the functional groups in the capillaries depending on their size and polarity.

The pore spacing (or capillary width) was estimated by the difference between the interlayer $\mathrm{d}$ spacing measured from XRD and the thickness of a single-layer of $\mathrm{MoS}_{2}$ : $6.15 \AA$ (c/2, with c the lattice parameter of $\left.2 \mathrm{H} \mathrm{MoS}_{2}\right)$. Our approach is thus similar to previous work from Abraham et al. on graphene oxide ${ }^{10}$. Using molecular dynamic simulations, we report water density profiles in the direction perpendicular to the flux inside 2D channels of $12 \AA$ separation (Supplementary Figure 15). Our molecular dynamic results show that water molecules do not get closer to sulfur atoms than $1.45 \AA$ giving a hydrodynamic thickness of $5.57 \AA$. The space that is inaccessible to water molecules is estimated to be: $12-5.57=6.43 \AA$ - close to the $6.15 \AA$ used for the study. 


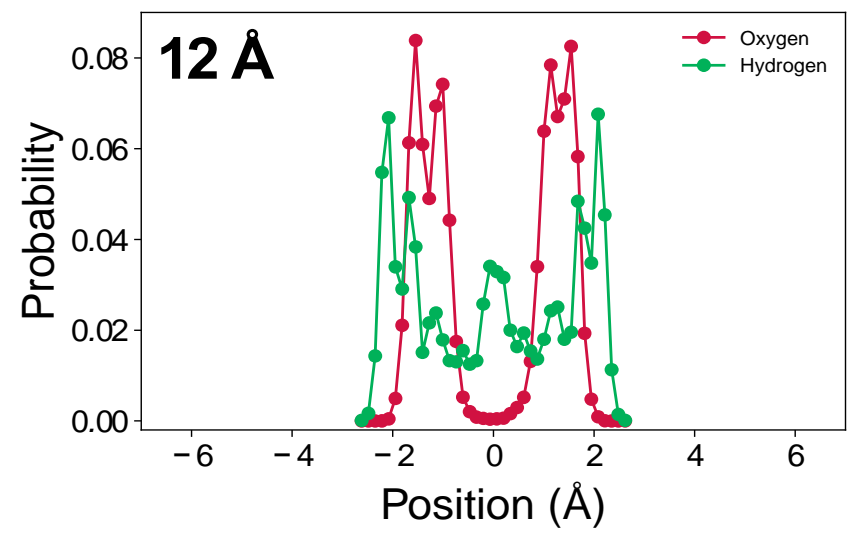

Supplementary Figure 15. Water density profile for $\mathrm{O}$ and $\mathrm{H}$ along the Y-direction for pristine $\mathrm{MoS}_{2}$ nanochannel with a 12 Å separation.

Supplementary Table 3. Comparison of lamellar structures of GO and $\mathrm{MoS}_{2}$ derived membranes. Capillary width of $\mathrm{GO}$ and $\mathrm{MoS}_{2}$-based membranes were estimated by considering the thickness of pristine graphene and $\mathrm{MoS}_{2}$ nanosheets: $3.4 \AA$ and $6.15 \AA$ respectively ${ }^{8}$. Errors correspond to standard deviation between several samples.

\begin{tabular}{|c|c|c|c|}
\hline Samples & $\begin{array}{c}\text { Interlayer } \\
\text { spacing d }(\AA)\end{array}$ & $\begin{array}{c}\text { Average } \\
\text { FWHM of }(002) \text { peak }\end{array}$ & $\begin{array}{c}\text { Capillary } \\
\text { width } \delta(\AA)^{*}\end{array}$ \\
\hline Graphite & 3.4 & - & - \\
\hline GO & $8.3( \pm 0.07)$ & 0.3 & 4.9 \\
\hline Bulk $\mathrm{MoS}_{2}$ powder & 6.14 & - & - \\
\hline Exfoliated $\mathrm{MoS}_{2}$ & $6.18( \pm 0.03)$ & 1.35 & 0.035 \\
\hline MoS $_{2}$-Acet. & $10.1( \pm 0.07)$ & 0.6 & 3.93 \\
\hline MoS $_{2}$-Met. & $11.5( \pm 0.02)$ & 0.82 & 5.37 \\
\hline $\mathrm{MoS}_{2}$-EtOH & $10.8( \pm 0.04)$ & 0.64 & 4.69 \\
\hline
\end{tabular}

\section{Stability of the functionalized $\mathrm{MoS}_{2}$ membranes}

Graphene oxide membranes are typically found unstable in water due to uncontrollable swelling. In order to assess the stability of the $\mathrm{MoS}_{2}$ membranes, the 
membranes were soaked in water for a period of one month. No visible macroscopic degradations are visible on the membranes as shown in Supplementary Figure 16.
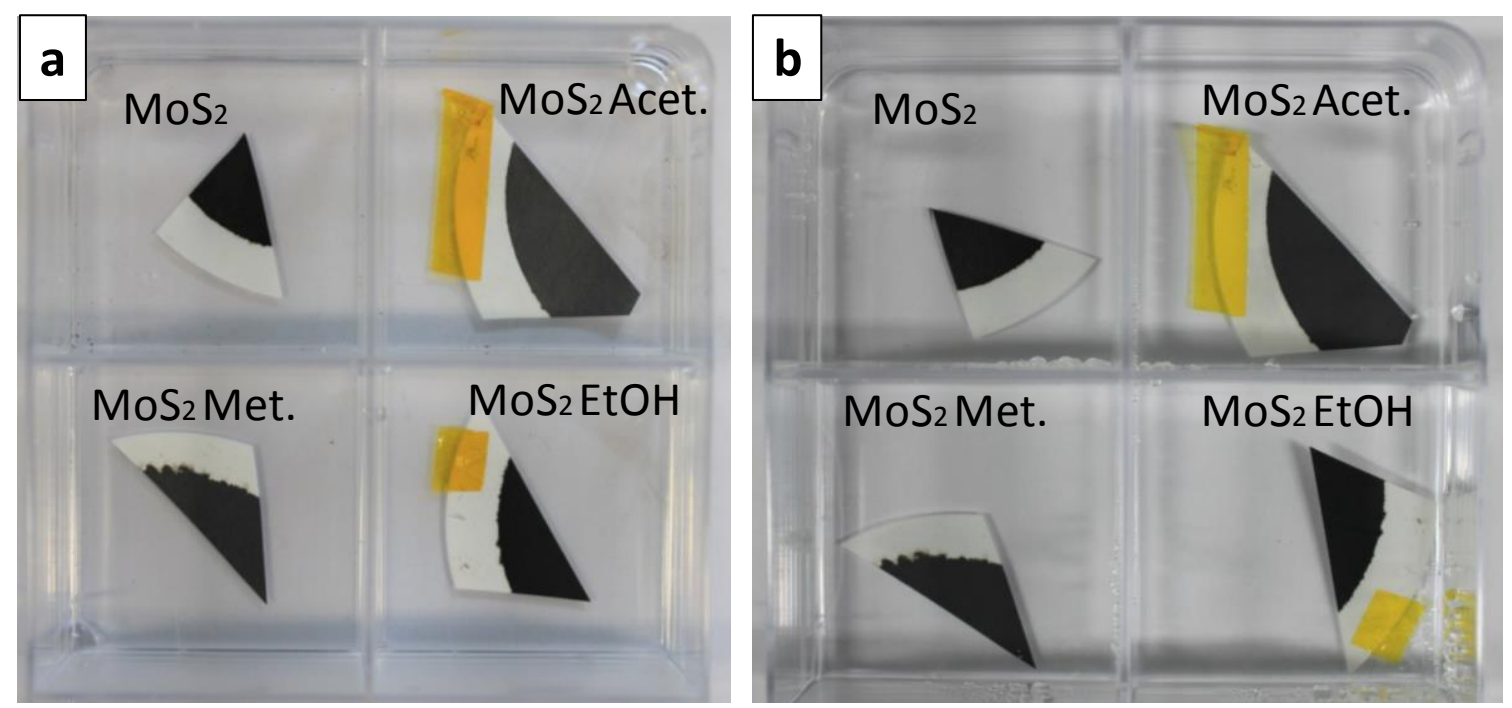

Supplementary Figure 16. Pictures of pristine and functionalized $\mathrm{MoS}_{2}$ membranes before (a) and after several months in DI water (b).

\section{Controlled of the swelling in the functionalized $\mathrm{MoS}_{2}$-based membranes}

In order to confirm the absence of deformation of the $\mathrm{MoS}_{2}$ membranes, the interlayer spacing was measured using X-ray diffraction after soaking the membrane in DI water. Supplementary Figure 17 shows the diffractograms of the $\mathrm{MoS}_{2}$ membranes compared to graphene oxide membranes for different soaking times. 

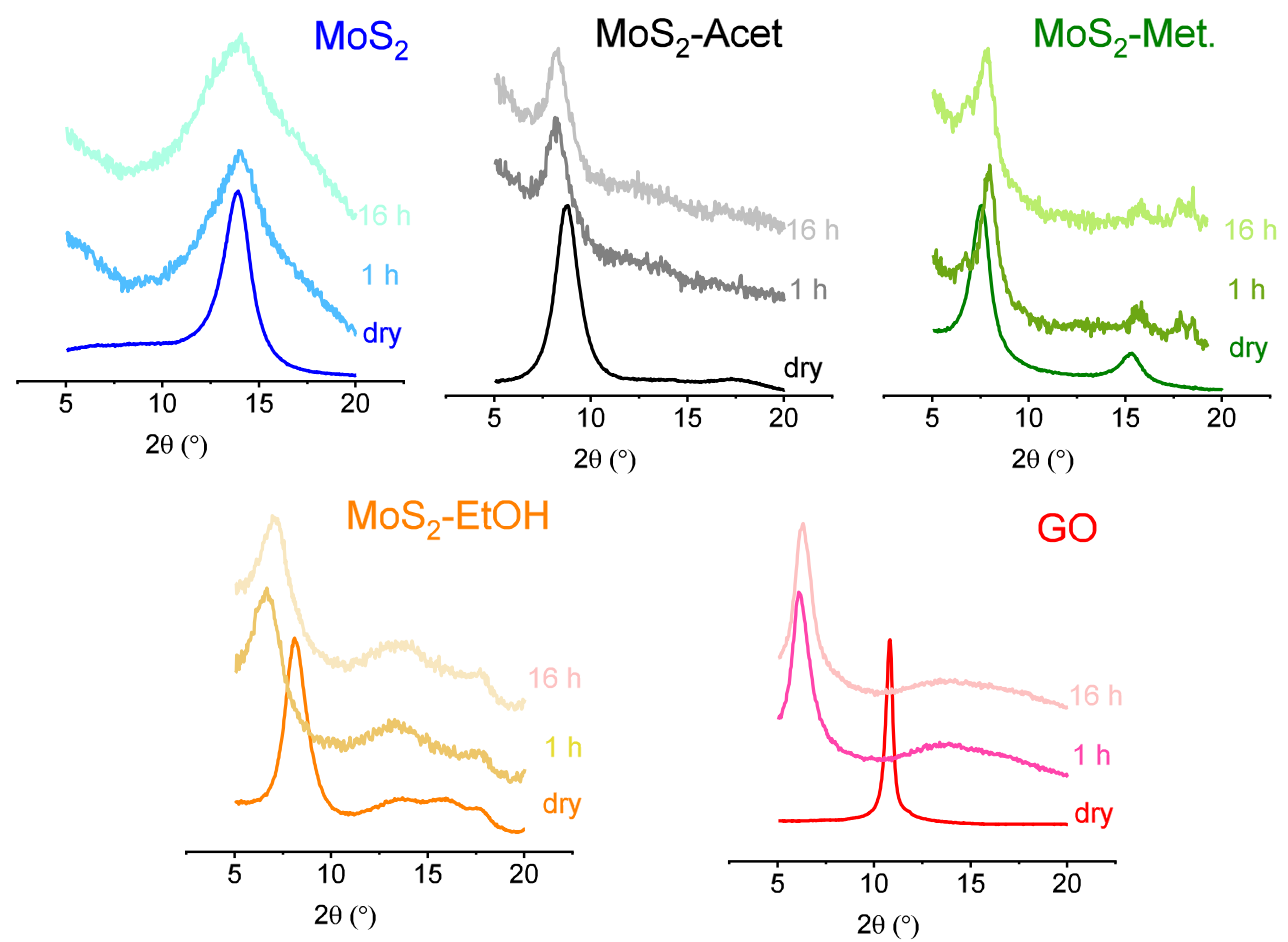

Supplementary Figure 17. X-ray diffraction spectra of $1 \mu \mathrm{m}$ thick $\mathrm{MoS}_{2}$ membranes dried and after 1 hour and 16 hours.

Next we investigated the consequences of hydration of the membrane on the (002) of the methyl- functionalized $\mathrm{MoS}_{2}$ membranes with different thicknesses. Supplementary Figure 18 shows the XRD spectra of $\mathrm{MoS}_{2}$-Met. membranes after 1h, $6 \mathrm{~h}$ and $48 \mathrm{~h}$ soaked in water. Our results show that the $\mathrm{MoS}_{2}$-Met. membranes do not swell and the broadening of the (002) peak is virtually not affected. We attributed this phenomenon to the hydrophobic nature of methyl groups. 

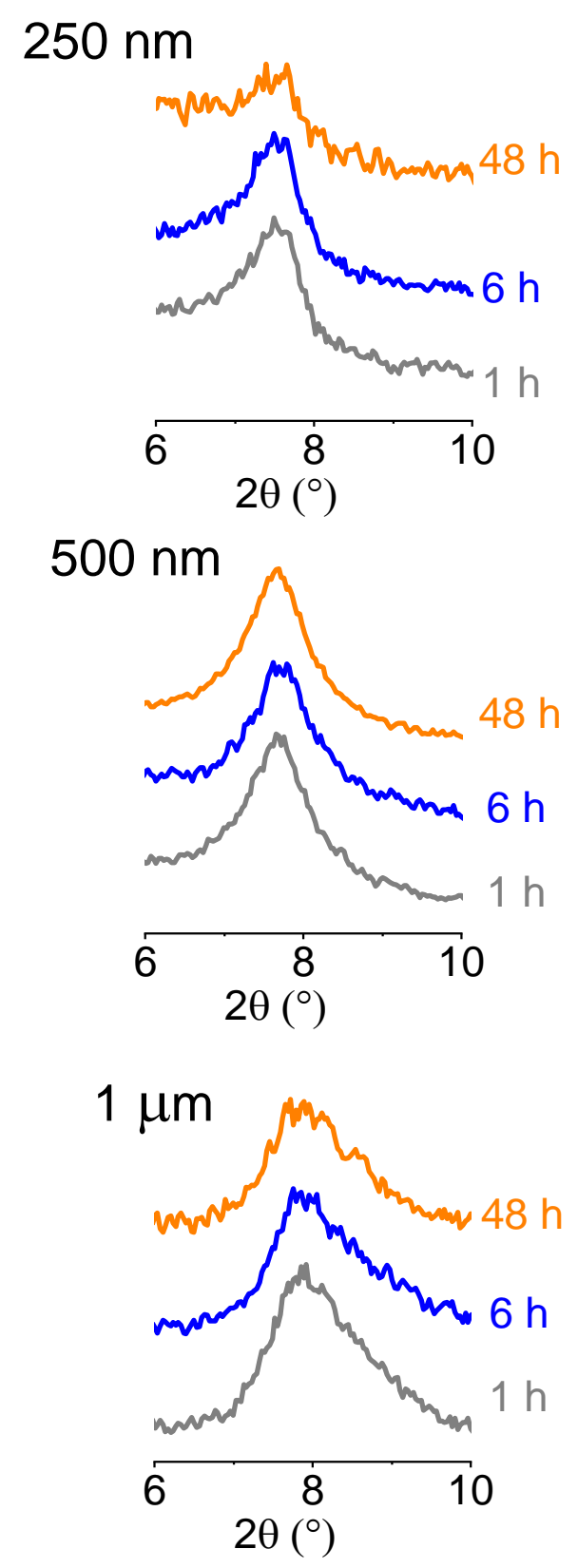

Supplementary Figure 18: X-ray diffraction spectra from hydrated $\mathrm{MoS}_{2}$-Met. membrane soaked in water for $1 \mathrm{~h}, 6 \mathrm{~h}$ and $48 \mathrm{~h}$. 


\section{Ion permeance measurements}

As discussed in the main text, ionic sieving properties have been examined. The ionic species tested in this work include $\mathrm{NaCl}, \mathrm{LiCl}$ and $\mathrm{KCl}$. We note that the anion is the same for all the salts so that the difference between the ion permeation rates is assumed to be solely due to cations. Ion permeation rate measurements were carried out in two Teflon-made compartments using $500 \mathrm{~nm}$-thick membranes and the 2 compartments were covered with aluminum foil in order to prevent water evaporation. Both permeate and feed reservoirs were filled with the same volume of $200 \mathrm{~mL}$ of DI water and aqueous ionic solution respectively (Supplementary Figure 19a). Ion transportation has been measured by recording the conductivity of the permeate solution over several hours. The size of the membrane was typically $1 \mathrm{~cm}^{2}$ in order to test at least 4 membranes from the same batch. To assess the transport properties, we performed the measurements for different ionic strengths corresponding to salt concentration of $0.25 \mathrm{M}, 0.625 \mathrm{M}$ and $1 \mathrm{M}$ in the feed side. As an example, Supplementary Figure 19b shows the permeation of $\mathrm{NaCl}$ across both acetamide- and ethyl-2-ol- functionalized $\mathrm{MoS}_{2}$ membranes using $1 \mathrm{M}$ of $\mathrm{NaCl}$ as feed compartment.

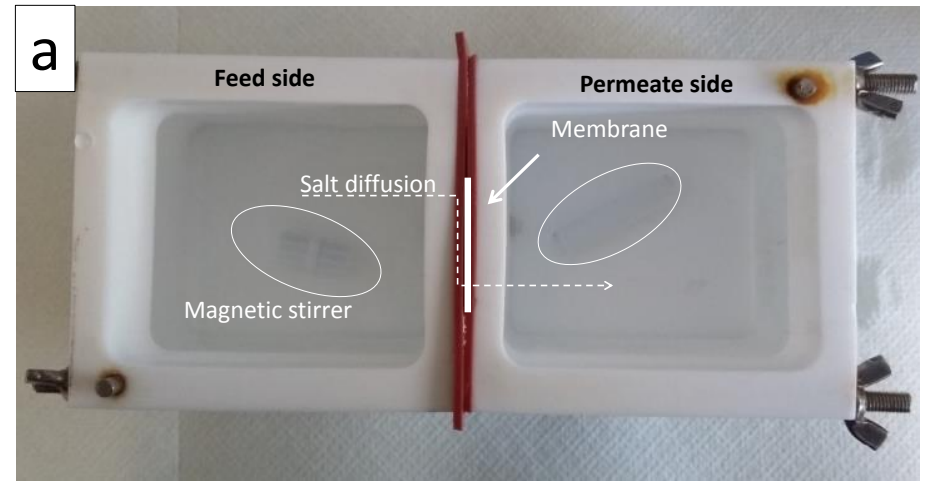




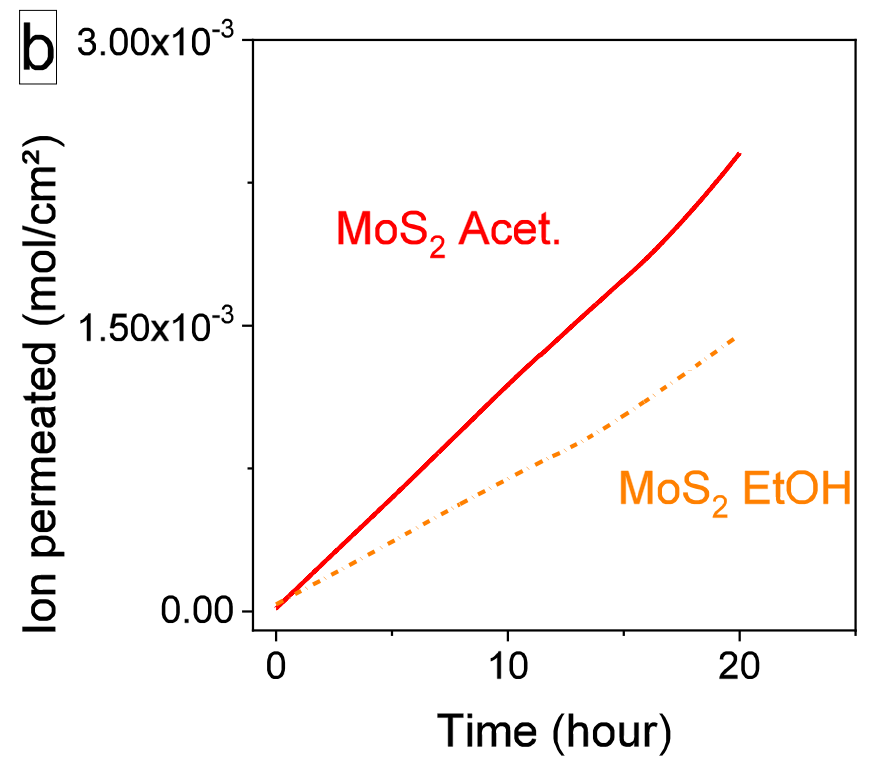

Supplementary Figure 19. Cell used for the conductivity measurement consisting of two compartments with the feed and permeate solutions (a) and evolution of the ion permeation across acetamide- (red line) and ethyl-2-ol- (orange line) functionalized membranes using a $1 \mathrm{M} \mathrm{NaCl}$ aqueous solution as feed solution (b).

The impact of the functional groups on the sieving mechanisms of several salts have been further investigated (Supplementary Figure 20). Hydrated ion diameters corresponding to cations only were obtained from $\operatorname{Ref}{ }^{10}: 6.6 \AA, 7.1 \AA$ and $7.6 \AA$ for $\mathrm{K}^{+}$, $\mathrm{Na}^{+}$, and $\mathrm{Li}^{+}$respectively. In the case of acetamide- functionalized membranes, ion permeance rates do not significantly change for all tested species at concentrations of $0.25 \mathrm{M}$ and $0.625 \mathrm{M}$. Overall the salt diffusion for small monovalent ions across acetamide-functionalized $\mathrm{MoS}_{2}$ membranes is rather independent of the size of the ions. The situation is different in the case of ethyl-2-ol-functionalized membranes, for which the ion permeance rate appears to be more controlled by the size of the ions (for concentrations $\geq 0.625 \mathrm{M}$ ) as previously seen in the case of graphene oxide membranes ${ }^{16}$. This result further demonstrates the influence of the surface chemistry for tuning the 
ionic diffusion in the 2D nanocapillaries.
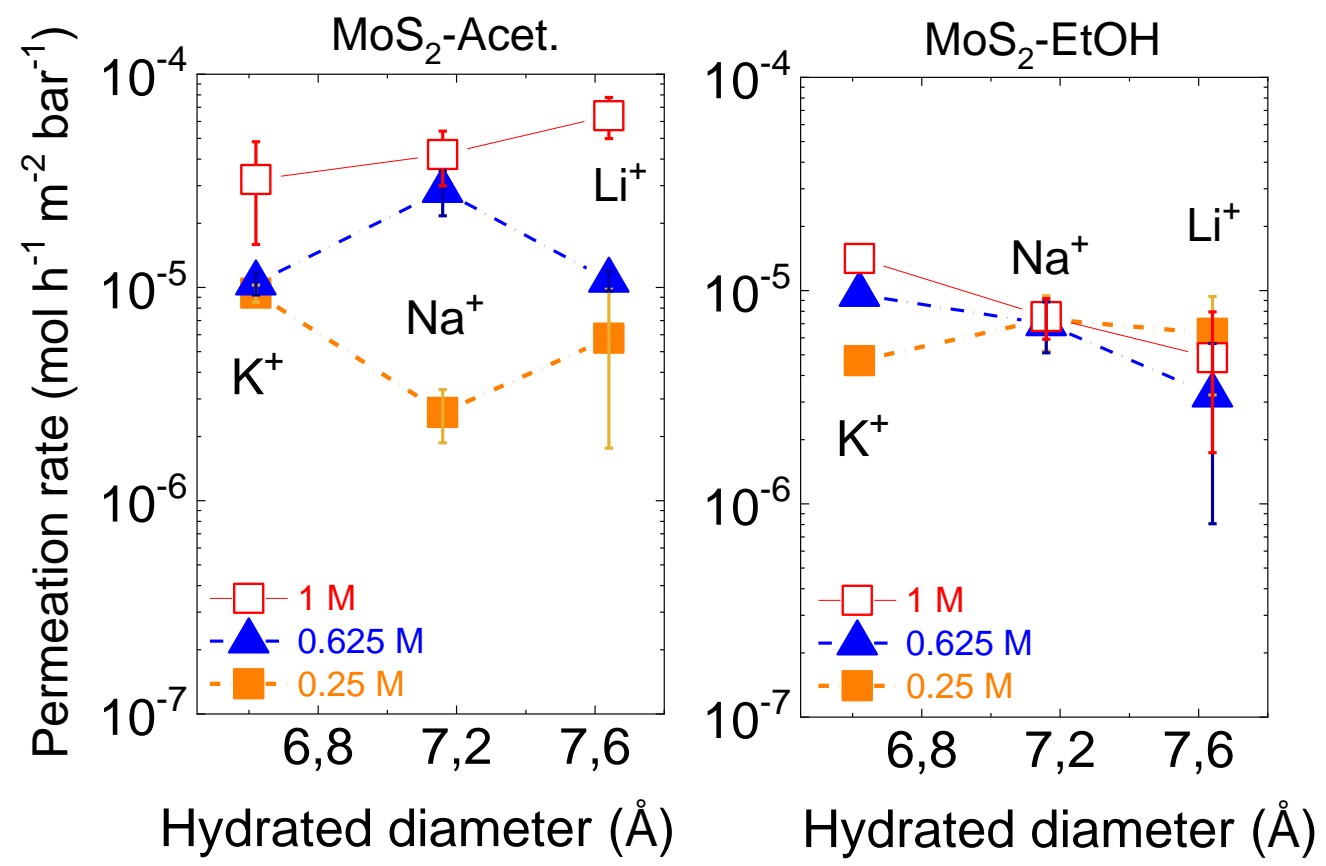

Supplementary Figure 20. Permeation rates of $\mathrm{Na}^{+}, \mathrm{Li}^{+}$and $\mathrm{K}^{+}$across acetamidefunctionalized (left) and ethyl-2-ol- functionalized (right) $\mathrm{MoS}_{2}$ membranes measured at different ionic strengths. Chloride was used as counter ion in all the cases.

\section{Permeation rate calculations}

Classical law of diffusion allows estimating the permeation rate of ions within the nanochannels. We assume that capillaries width of the structures allow the diffusion of all tested species without considering their hydrated diameters as a limiting factor. The permeation rate $\mathrm{J}$ is given by Equation (1):

$$
\mathrm{J}=\frac{D \times \Delta C \times A_{e f f}}{L_{e f f}}
$$

where $\mathrm{D}$ is the diffusion coefficient for ions in water $\left(\mathrm{D} \approx 10^{-5} \mathrm{~cm}^{2} / \mathrm{s}\right.$ for small ions $\left.{ }^{11}\right)$, $\Delta \mathrm{C}$ is the concentration gradient, $\mathrm{A}_{\mathrm{eff}}$ is the total cross-sectional area of nanochannels 
and $\mathrm{L}_{\text {eff }}$ the diffusion length through the $\mathrm{MoS}_{2}$-based membranes. For a $1 \mu \mathrm{m}$ thick membrane with an area of $1 \mathrm{~cm}^{2}$, the effective area through which the diffusion can occur, the ionic permeation rates can be obtained using Equations (2,3);

$$
\begin{gathered}
A_{e f f} \approx 1 \mathrm{~cm}^{2} \times \frac{d}{L} \\
L_{e f f} \approx L \times \frac{h}{d}
\end{gathered}
$$

where $\mathrm{L}$ is the average lateral length of functionalized $\mathrm{MoS}_{2}$ sheets $(\approx 340 \mathrm{~nm})^{12}$.

Supplementary Table 4. Estimation of fluxes of small ions using the classical law of

\begin{tabular}{|c|c|c|c|}
\hline Membrane & $A_{\text {eff }}\left(\mathbf{m m}^{2}\right)$ & $\mathbf{L}_{\text {eff }}(\mathbf{m m})$ & $\mathrm{J} \times 10^{-4}\left(\mathrm{~mol} \cdot \mathrm{h}^{-1} \cdot \mathrm{m}^{-2} \cdot \mathrm{bar}^{-1}\right)$ \\
\hline $\mathrm{MoS}_{2}$-Acet. & 0.36 & 0.28 & 3.15 \\
\hline $\mathrm{MoS}_{2}-\mathrm{EtOH}$ & 0.36 & 0.28 & 3.21 \\
\hline
\end{tabular}
diffusion (considering $1 \mathrm{M}$ concentration gradient).

\section{Binding energy between the ions and the functional groups}

As the capillary width is typically lower than the hydrated diameters of the ions, the ions must - at least partially - dehydrate to permeate the membrane. The ion permeation for ethyl-2-ol- functionalized $\mathrm{MoS}_{2}$ membranes increases with the decrease of the dehydration energy (Supplementary Figure 21a). The binding energy between the ions and the functional groups has been estimated using DFT calculations (Supplementary Table 5). We define the binding energy as the energy of the ion adsorbed on the functional group minus the energy of the functional group minus the energy of the ion. We found that the ions do not adsorbed on the $\mathrm{NH}_{2}$ site of the acetamide group but rather 
move to the O-site after geometry optimization. In the case of ethyl-2-ol group, the ions adsorbed on both $\mathrm{O}$-site and $\mathrm{CH}_{2}$, although the energy of the latter is relatively small. DFT calculations were performed with ORCA ${ }^{13}$ at the B3LYP level and with the def2TZVP basis set.

Supplementary Table 5. Binding energy between $\mathrm{Na}^{+}, \mathrm{Li}^{+}, \mathrm{K}^{+}$and $\mathrm{Cl}^{-}$and the different functional groups.

\begin{tabular}{|c|c|c|c|c|}
\hline & & \multicolumn{4}{|c|}{$\mathbf{B E}(\mathbf{e V})$} \\
\hline Ions & Acet. (-O site) & EtOH (-CH2 site) & EtOH(-O sites) & Met. \\
\hline $\mathrm{Li}^{+}$ & -2.42 & -0.44 & -1.8 & -0.53 \\
\hline $\mathrm{Na}^{+}$ & -1.72 & -0.19 & -1.25 & -0.3 \\
\hline $\mathrm{K}^{+}$ & -1.27 & -0.04 & -0.87 & -0.15 \\
\hline $\mathrm{Cl}^{-}$ & -1.09 & -0.35 & $n / a$ & -0.18 \\
\hline
\end{tabular}

Our results demonstrate that $\mathrm{Li}^{+}$has a stronger affinity with acetamide and ethy-2-ol functions than $\mathrm{Na}^{+}$and $\mathrm{K}^{+}$and the interaction sites are located at the oxygen sites of the amide and alcohol groups, respectively. Supplementary Figure 21 show the permeation rates for acetamide and ethyl-2-ol- functionalized $\mathrm{MoS}_{2}$ membranes as function of the binding energy (Supplementary Table 5). One can see that the behavior of the functionalized $\mathrm{MoS}_{2}$ membranes depends on the nature of the functional groups. In the case of $\mathrm{MoS}_{2}-\mathrm{EtOH}$ membranes, we found that the permeation rates increase for lower values of the binding energy, suggesting that the electrostatic interactions between the ions and the functional groups play an important role on the controlled diffusion of ions across the membrane: the weaker the interactions, the higher the ion permeation rates 
(Supplementary Figure 21c). This is also supported by our Zeta potential measurements that reveal that the $\mathrm{MoS}_{2}$ nanosheets are charged (Supplementary Table 1). The ion transport mechanism in functionalized $\mathrm{MoS}_{2}$ membranes is thus similar to previously reported mechanism for the permeation of ions in graphene oxide membranes, where the ion permeation is also controlled by the electrostatic interactions with the charges carried by the nanosheets ${ }^{14}$. On the contrary, in the case of membranes made of acetamidefunctionalized $\mathrm{MoS}_{2}$ nanosheets, the permeation rates appear to mainly depend on the ionic strength (Supplementary Figure 20 and main Figure 3a), whereas the binding energy and the dehydration energy do not seem to significantly influence the ion diffusion in the nanolaminate membranes (Supplementary Figure 21). The apparent different behavior between $\mathrm{MoS}_{2}-\mathrm{EtOH}$ and $\mathrm{MoS}_{2}$-Acet. membranes is attributed to the reduced capillary width: $4.6 \AA$ for $\mathrm{MoS}_{2}$-EtOH compared to $3.9 \AA$ for $\mathrm{MoS}_{2}$-Acet. and the larger binding energy between the ions and the acetamide functional group. The larger capillary widths of $\mathrm{MoS}_{2}$-EtOH membranes allow faster diffusion of the ions and thus the interactions between the ions and functional groups are likely to play a more important role on the ion permeation. At the oppostite, the ion permeation is strictly controlled by the narrower capillary width of $\mathrm{MoS}_{2}$-Acet. and the permeation is influenced by the ionic strength of the solution.

Supplementary Table 6. Summary of the hydrated diameter, binding energy between $\mathrm{Na}^{+}, \mathrm{Li}^{+}$and $\mathrm{K}^{+}$and the different functional groups compared with the permeation rates measured at $0.625 \mathrm{M}$. The dehydration energies are obtained from Ref. ${ }^{15}$.

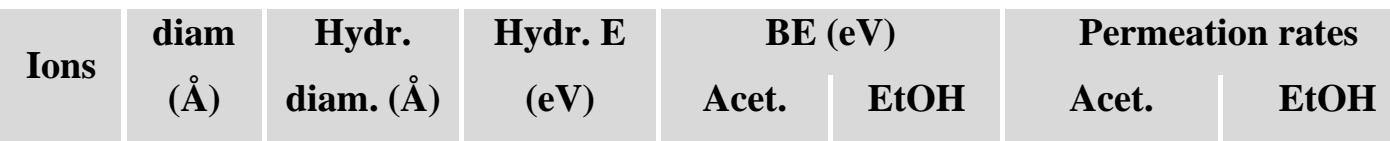




\begin{tabular}{|c|c|c|c|c|c|c|c|}
\hline $\mathrm{Li}^{+}$ & 1.36 & 7.6 & 5.38 & -2.42 & -1.8 & $1.07 \times 10^{-5}$ & $3.23 \times 10^{-6}$ \\
\hline $\mathrm{Na}^{+}$ & 1.9 & 7.1 & 4.24 & -1.72 & -1.25 & $2.84 \times 10^{-5}$ & $6.98 \times 10^{-6}$ \\
\hline $\mathrm{K}^{+}$ & 2.66 & 6.6 & 3.34 & -1.27 & -0.87 & $1.04 \times 10^{-5}$ & $9.58 \times 10^{-6}$ \\
\hline
\end{tabular}
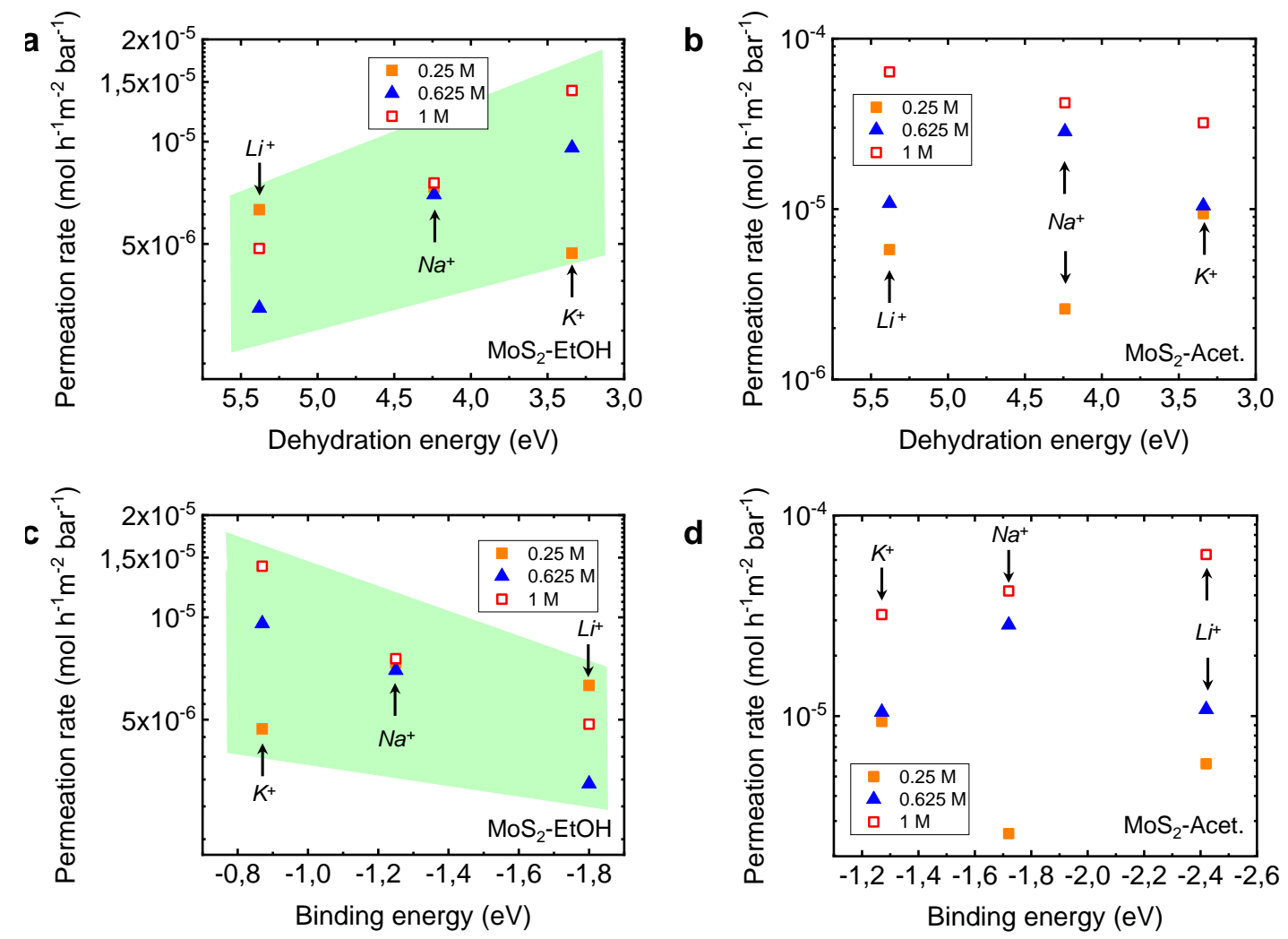

Supplementary Figure 21. Evolution of the permeation rates for $\mathrm{MoS}_{2}-\mathrm{EtOH}$ and $\mathrm{MoS}_{2}$ Acet. measured in forward osmosis configuration with the dehydration energy (a,b) and the binding energy $(\mathbf{c}, \mathbf{d})$ for $\mathrm{Na}^{+}, \mathrm{Li}^{+}, \mathrm{K}^{+}$and $\mathrm{Cl}^{-}$with ionic strengths of $0.25 \mathrm{M} ; 0.625 \mathrm{M}$ and $1 \mathrm{M}$.

\section{Forward osmosis (FO)-driven measurements}

Water flux across the $\mathrm{MoS}_{2}$ membranes has also been evaluated in forward osmosis configuration. Measurements have been performed using a custom-made cell presented in Supplementary Figure 22, wherein membranes were clamped between a feed and a draw glass reservoirs filled with $0.1 \mathrm{M} \mathrm{NaCl}$ and $3 \mathrm{M} \mathrm{KCl}$ solution in order to generate forward 
osmosis pressure of $\sim 148$ bar. The size of the membrane was typically $1 \mathrm{~cm}^{2}$ in order to test at least 4 membranes from the same batch. We also fabricated and tested $\mathrm{MoS}_{2}$ membranes from different batches to confirm the reproducibility of the results. All the membranes were tested using Nylon as porous substrate with 220 -nm pore size. The water flux was directly measured from the increase of the water meniscus in the cell (yellow arrows in Supplementary Figure 22). The detection limit of the set-up is approximated to $0.01 \quad \mathrm{~L} \cdot \mathrm{h}^{-1} \cdot \mathrm{m}^{-2} \cdot \mathrm{bar}^{-1}$ considering our experimental conditions. Interestingly methyl- functionalized $\mathrm{MoS}_{2}$ membranes do not show any water flow under forward osmosis. The suppression of the water permeance in forward osmosis in the case of methyl- functionalized $\mathrm{MoS}_{2}$ is attributed to the hydrophobic nature of the nanosheets that does not allow water to penetrate the 2D capillaries unless sufficient external pressure is supplied to the system ${ }^{16}$. These membranes are thus not discussed in the following section.

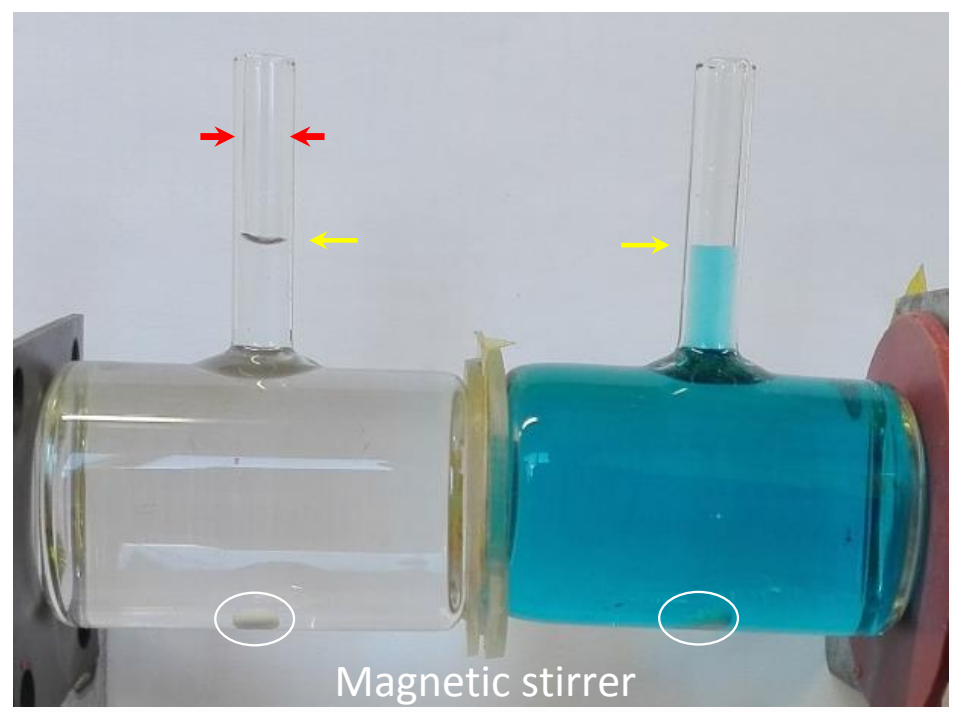

Supplementary Figure 22. Custom-made cell used for the forward osmosis measurements with the feed and draw reservoir containing the Ni-phtalocyanine solution 
(right) and the $3 \mathrm{M} \mathrm{KCl}$ draw solution (left) respectively. The water meniscuses in both compartments are shown with the yellow arrows. The red arrows indicate the $5 \mathrm{~mm}$ diameter cylinders used for measuring the water diffusion.

Supplementary Figure 23 presents the water flow rates obtained via osmosisdriven pressure for the different functionalized $\mathrm{MoS}_{2}$ membranes. We found that water cannot permeate in the non-functionalized $\mathrm{MoS}_{2}$ membranes in perfect agreement with the reversed osmosis results. Ethyl-2-ol- functionalized $\mathrm{MoS}_{2}$ membranes display the highest water permeance compared to the acetamide- functionalized $\mathrm{MoS}_{2}$ membranes. The permeance is also larger than previous results from the literature for membranes made of exfoliated-restacked nanosheets. Interestingly methyl- functionalized $\mathrm{MoS}_{2}$ membranes do not show any water flow under forward osmosis. The suppression of the water permeance in forward osmosis in the case of methyl- functionalized $\mathrm{MoS}_{2}$ is attributed to the hydrophobic nature of the nanosheets that does not allow water to penetrate the $2 \mathrm{D}$ capillaries unless sufficient external pressure is supplied to the system. 


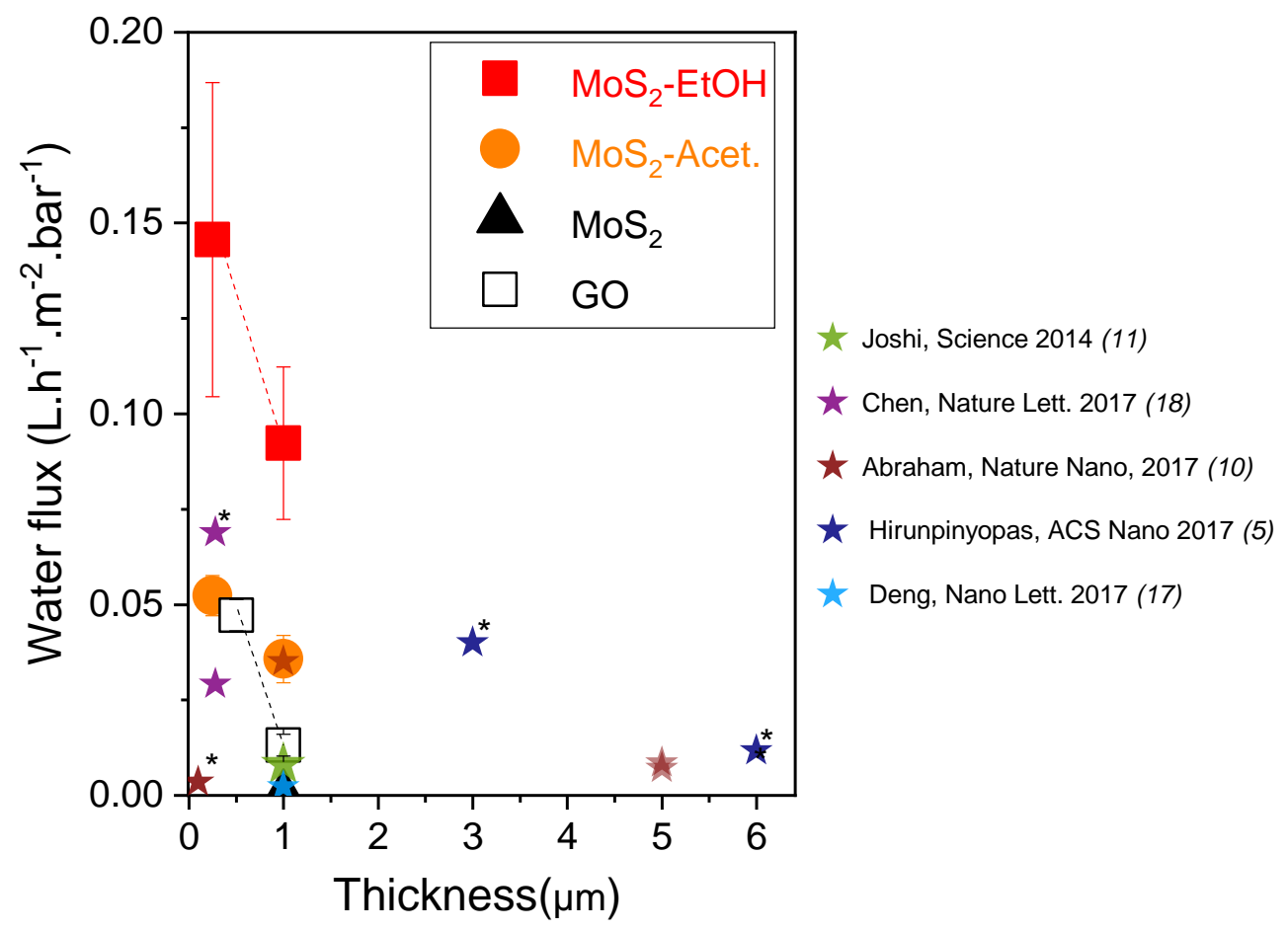

Supplementary Figure 23. Water permeance performances of several 2D-based membranes using osmotically-driven pressure. Water flow for the functionalized $\mathrm{MoS}_{2}$ membranes is compared with the performance of graphene oxide membranes prepared at the laboratory (open squares) and references from the literature (stars) $5,10,11,17,18$

\section{Reverse osmosis (RO) measurements}

The performances of the membranes towards water permeance were characterized by measuring the amount of water crossing the membranes under increasing pressure. The different membranes have been tested using a polysulfone cell (Amicon@) in dead-end configuration under pressures up to 4 bars. The size of the membrane was typically $1 \mathrm{~cm}^{2}$ in order to test at least 4 membranes from the same batch. We also fabricated and tested $\mathrm{MoS}_{2}$ membranes from different batches in order to confirm the reproducibility of the results. Prior any measurements, the flow of water across the membranes was stabilized 
under 4 bars for $1 \mathrm{~h}$. Typical water flux profile under increasing pressure is depicted in Supplementary Figure 24. The linear fit allows determining water flux (in $\mathrm{L} \mathrm{m}^{-2} \mathrm{~h}^{-1} \mathrm{bar}^{-1}$ ) of membranes (main Figure 3c).

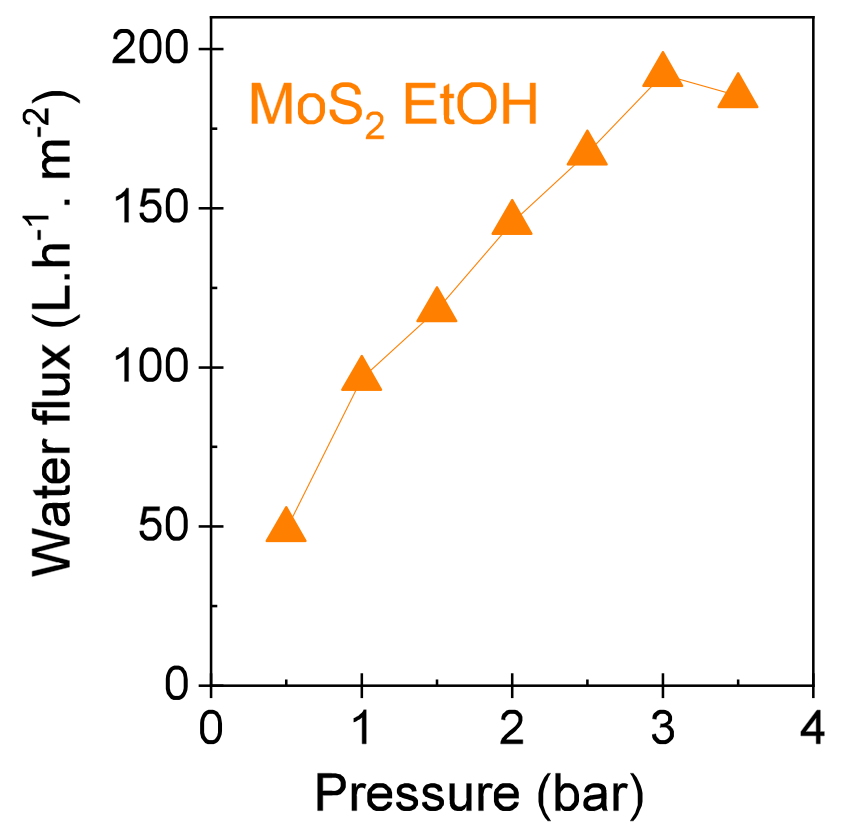

Supplementary Figure 24. Typical water flux profile obtained for ethyl-2-olfunctionalized $\mathrm{MoS}_{2}$ membranes with a thickness $250 \mathrm{~nm}$.

Interestingly the high water flux across methyl- functionalized $\mathrm{MoS}_{2}$ membranes is maintained when increasing the thickness up to $4 \mu \mathrm{m}$. Supplementary Figure 25 shows the trend of water flux with thickness in methyl- and ethyl-2-ol- functionalized $\mathrm{MoS}_{2}$ membranes. The inset shows the comparison between the water fluxes for $\mathrm{MoS}_{2}-\mathrm{Met}$. and $\mathrm{MoS}_{2}$-EtOH membranes up to $2 \mu \mathrm{m}$ in thickness. According to our molecular dynamic simulations (Supplementary Figure 43), the functionalization degree affects the water flux however, the few percent differences in functionalization degrees measured by XPS for the $\mathrm{MoS}_{2}$-Met. cannot explain the untypical water flux of the membrane with 
increased thickness (Supplementary Figure 27.b). By contrast, the distribution of $d$ spacing within the membrane can largely affect the water flux as suggested by the simulations performed on pristine membranes (Figure $4 \mathrm{~b}$ in the main text) for which a 2 $\AA$ variation in d-spacing results (from $10 \AA$ to $12 \AA$ ) in a 10-fold increase of the water flux. The larger FWHM of the (002) XRD peak for $\mathrm{MoS}_{2}$-Met. compared to $\mathrm{MoS}_{2}$-Acet. may lead to larger water flux due to different stacking order of the nanosheets (Supplementary Figure 28). We believe that this last point combined with the hydrophobic nature of the methyl group is at the origin of the behavior of $\mathrm{MoS}_{2}$-Met. membranes compared to the $\mathrm{MoS}_{2}$-Acet and $\mathrm{MoS}_{2}-\mathrm{EtOH}$ membranes. The hydrophobicity of the methyl-functionalized $\mathrm{MoS}_{2}$ membranes also translates into nanocapillaries with larger slip length. The role of the hydrophobicity on the water flux has been previously identified in the case of carbon nanotubes, whose predicted water fluxes do not depend on the length of the tube ${ }^{19}$. We note that the similar behavior has been reported by Hirunpinyopas et al. for dye-decorated $\mathrm{MoS}_{2}$ membranes with a $\sim 9$ fold increase when the thickness increases from 1 to $6 \mu \mathrm{m}$ and attributed to the hydrophobic surface of $\mathrm{MoS}_{2}\left({ }^{5}\right)$. In the case of more hydrophilic membranes such as acetamide and ethyl-2-ol membranes, the water flux rapidly decreases as the thickness increases. We suspect compaction of the thinnest $\mathrm{MoS}_{2}$-Met.membrane because of the small size of the functional groups. The stiffness of the membrane should be explored in a follow-up study. . 


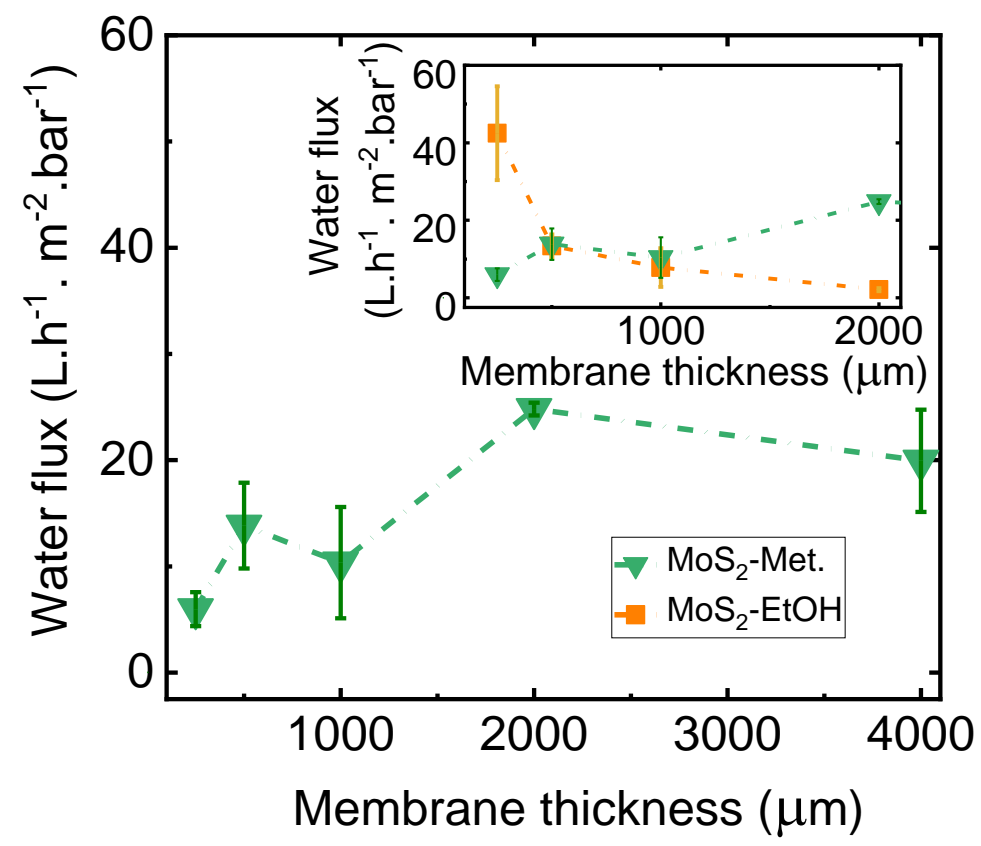

Supplementary Figure 25. Water flux for methyl-functionalized $\mathrm{MoS}_{2}$ membranes with thicknesses up to $4 \mu \mathrm{m}$. Inset: Comparison water flux in thick methyl- and ethyl-2-olfunctionalized $\mathrm{MoS}_{2}$ membranes.

\section{Stability of the water flux in dead-end configuration}

The stability of nanolaminate membranes is an important parameter and instability is typically observed for graphene oxide membranes. For example Chong et al. ${ }^{13}$ recently showed that the water flux for graphene oxide membranes decreases from 10 's $\mathrm{L} \mathrm{m}^{-2} \mathrm{~h}^{-1}$ bar $^{-1}$ to $0.1 \mathrm{~L} \mathrm{~m}^{-2} \mathrm{~h}^{-1}$ bar $^{1}$ after only $30 \mathrm{~min}$. We investigated the stability of methylfunctionalized $\mathrm{MoS}_{2}$ in dead-end configuration under a pressure of 4 bar for 15 hours. Supplementary Figure 26 shows that the water flux is largely preserved suggesting that the compaction effect is significantly reduced compared to graphene oxide membranes. 


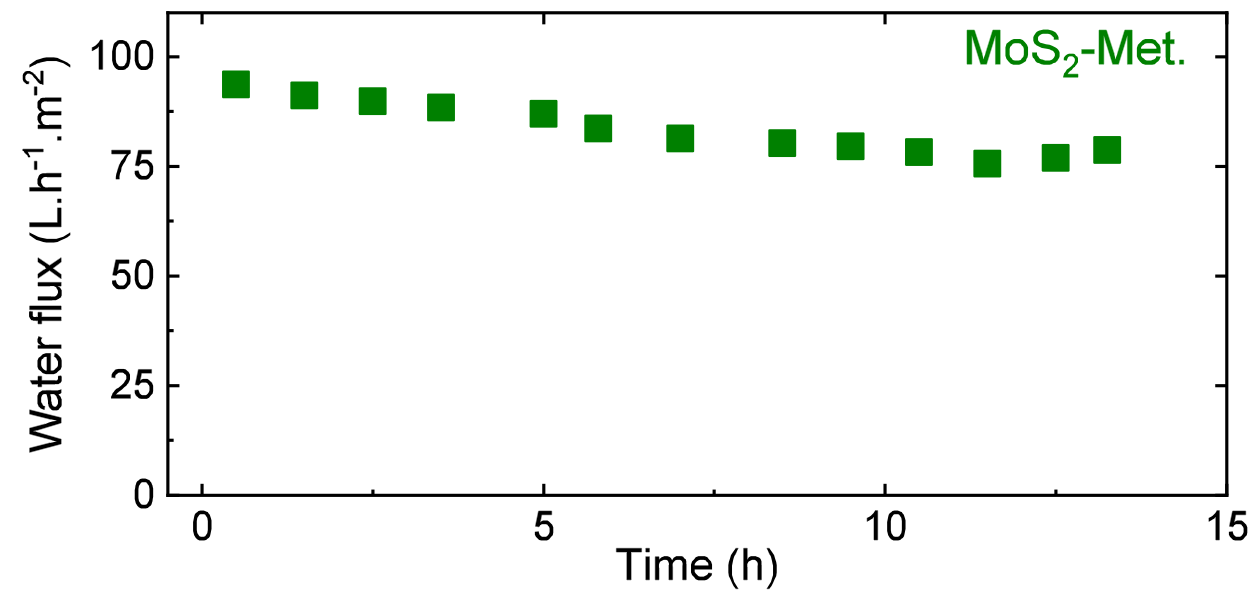

Supplementary Figure 26. Stability of the water flux for $500 \mathrm{~nm}$-thick methylfunctionalized $\mathrm{MoS}_{2}$ membrane in dead-end configuration with an applied pressure of 4 bar.

Influence of the membrane thickness on the functionalization degree and stacking order of the $\mathrm{MoS}_{2}$ nanosheets

The influence of the thickness on the structure of the functionalized $\mathrm{MoS}_{2}$ membranes were further analyzed using X-ray diffraction (XRD) and X-ray photoelectron spectroscopy (XPS). We first quantified the functionalization degree of $\mathrm{MoS}_{2}-\mathrm{Met}$. and $\mathrm{MoS}_{2}$-Acet. membranes with different thicknesses using XPS. We note that $\mathrm{MoS}_{2}$-Met. membranes were prepared via direct functionalization on the porous support whereas $\mathrm{MoS}_{2}$-acet. and $\mathrm{MoS}_{2}$-EtOH membranes were functionalized in solution prior filtration. The functionalization degrees of $\mathrm{MoS}_{2}$-acet. and $\mathrm{MoS}_{2}-\mathrm{EtOH}$ membranes are thus likely to be homogeneous. We quantified the extent of functional groups on the $\mathrm{MoS}_{2}$-Met. nanosheets at different membrane thicknesses. To do so, XPS was performed on the core of 4 different $\mathrm{MoS}_{2}$-Met. membranes with increasing thicknesses from $250 \mathrm{~nm}$ up to $2000 \mathrm{~nm}$ (). The XPS samples were prepared by mechanically pealing the membranes 
using adhesive tape. We deconvoluted the signals from the S2p region of each sample following the same method as described above (and Table 2). The signals of the S2p doublet ascribed to the S-C bond were used to quantify the functionalization degree. Our results show that the extent of functionalization per $\mathrm{S}$ atom does not significantly change when increasing the thickness of the membranes. The nanosheets are slightly more functionalized on the top surface compared to the core of the membrane and the functionalization degree varies from $\approx 25 \%$ down to $\approx 19 \%$ for the top surface and the $2000 \mathrm{~nm}$-thick membrane respectively. Importantly our measurements also reveal that the amount of grafted functional groups on the $\mathrm{MoS}_{2}$ nanosheets inside the membrane remains virtually constant for thicknesses as high as $2000 \mathrm{~nm}$.
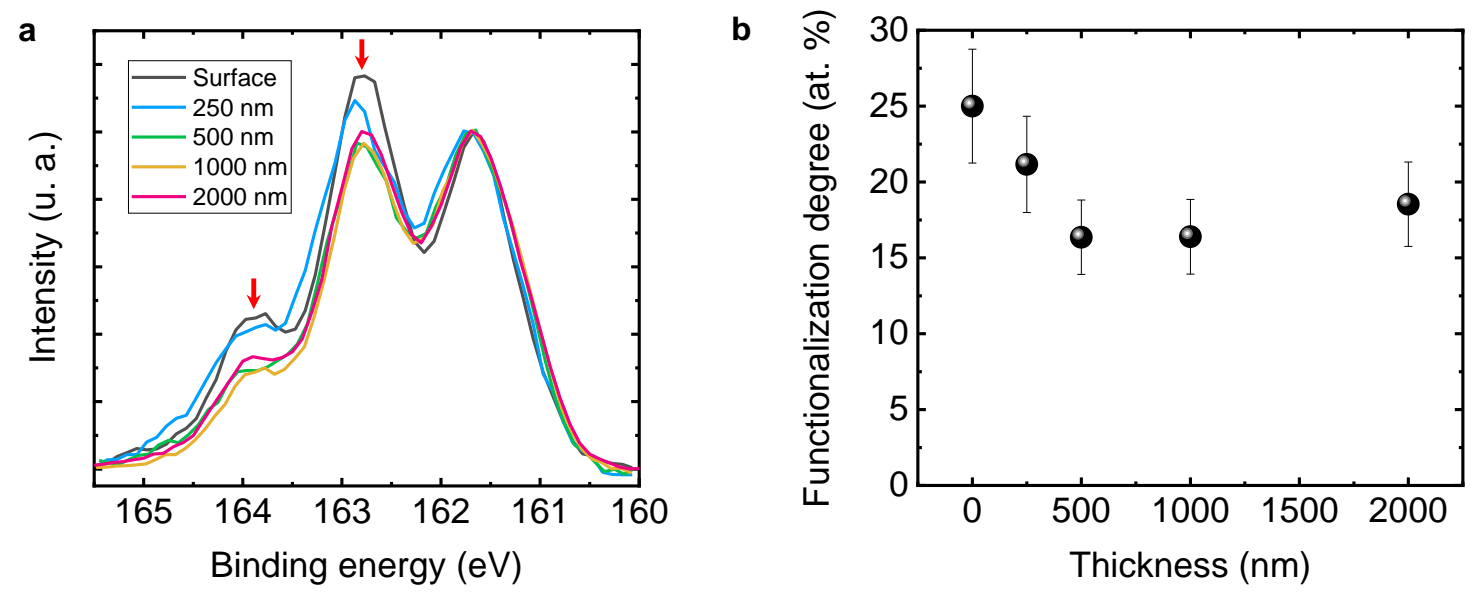

Supplementary Figure 27. a, Normalized high-resolution XPS spectra from the S2p region from $\mathrm{MoS}_{2}$-Met. membranes with increasing thicknesses from $250 \mathrm{~nm}$ up to 2000 $\mathrm{nm}$. $\mathbf{b}$, Evolution of the functionalization degree per $\mathrm{S}$ atom of $\mathrm{MoS}_{2}$-Met. membranes with thicknesses of $250 \mathrm{~nm}-2000 \mathrm{~nm}$. The functionalization degrees are estimated from the deconvolution of the S2p region.

shows the X-ray diffraction responses of membranes made of $\mathrm{MoS}_{2}$ nanosheets functionalized with acetamide and methyl groups. We found that for both types of 
membranes, the (002) peak is not significantly shifted; thus demonstrating that the interlayering spacing is largely preserved and independent of the membrane thickness. On the other hand, the full width at half maximum (FWHM) increases as the thickness increases from $250 \mathrm{~nm}$ up to $2000 \mathrm{~nm}$ suggesting that the degree of order of the vertical stacking of the $\mathrm{MoS}_{2}$ nanosheets in the membranes reduces with the increase of the thickness of the membranes $(a, b)$.

a

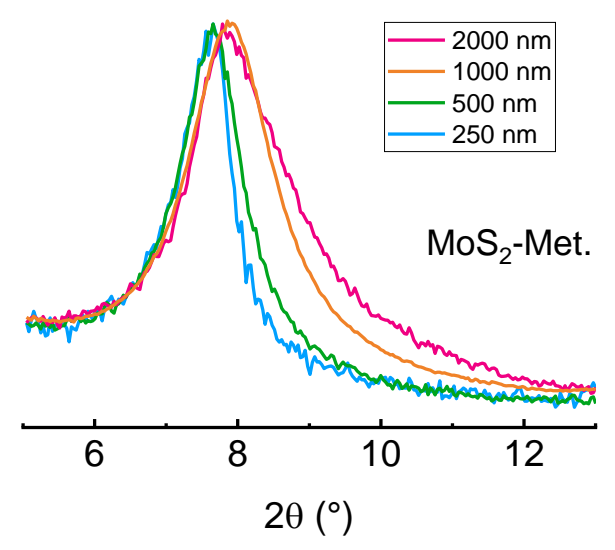

b

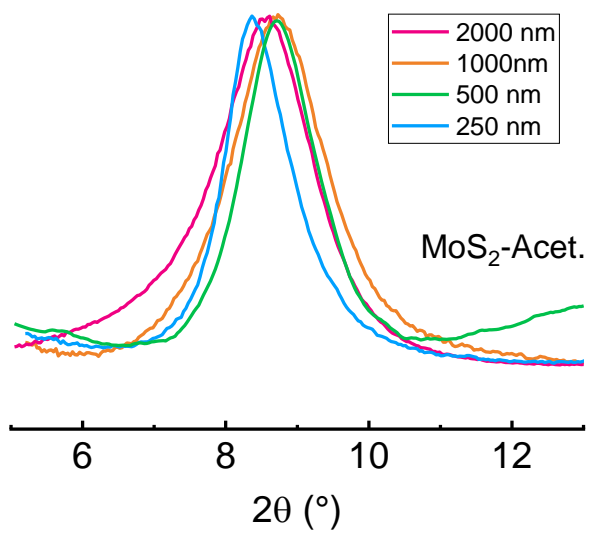

Supplementary Figure 28. Normalized X-ray diffraction spectra centered around of the (002) peak from the membranes made of methyl- (a) and acetamide- (b) functionalized MoS2 nanosheets with increasing thicknesses from $250 \mathrm{~nm}$ up to $2000 \mathrm{~nm}$.

We observed that the broadening of the (002) XRD peak for the $\mathrm{MoS}_{2}-$ Met. membranes deviates from the evolution of that of $\mathrm{MoS}_{2}$-acet. membranes for thicknesses larger than $1000 \mathrm{~nm}$ suggesting further disorder in the case of methyl- functionalized $\mathrm{MoS}_{2}$ nanosheets $(\mathrm{a}, \mathrm{b})$. We also analyzed the nanolaminate membranes made of ethyl-2ol- functionalized $\mathrm{MoS}_{2}$ nanosheets (prepared by the same method as for the $\mathrm{MoS}_{2}$-Acet. membranes) and the same trend as for $\mathrm{MoS}_{2}$-Acet. membranes was observed. 

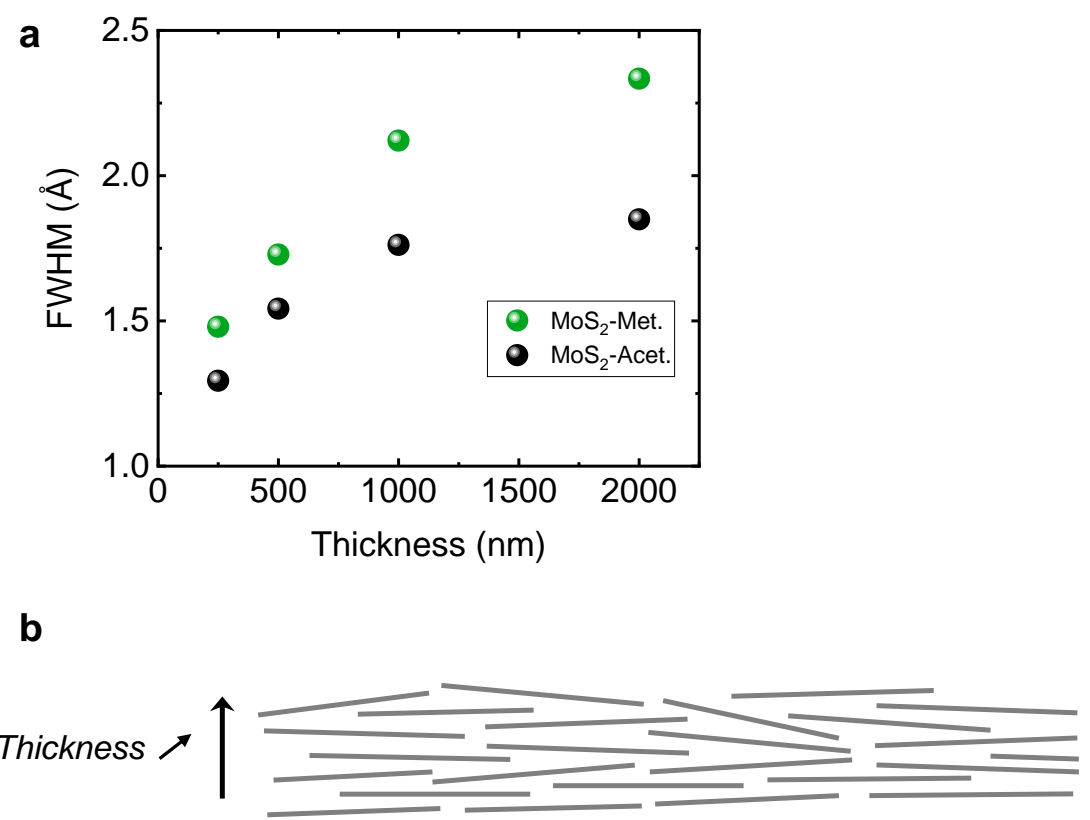

Supplementary Figure 29. a) Evolution of the full width at half maximum (FWHM) of the (002) peaks between $250 \mathrm{~nm}$ and $2000 \mathrm{~nm}$. b) Schematic showing the influence of the vertical stacking of the nanosheets on the broadening of the (002) XRD peak of the functionalized $\mathrm{MoS}_{2}$ membranes.

Interestingly the broadening of the (002) peak of $\mathrm{MoS}_{2}$-Acet. membranes is symmetrical, while an asymmetry is clearly observed for the (002) peak of $\mathrm{MoS}_{2}$-Met. (a,b). We attribute this phenomenon to a locally lower functionalization on the nanosheets likely due to a limited access of the reagents for the narrowest regions of the 2D capillaries. Indeed $\mathrm{MoS}_{2}$-Met. membranes are functionalized on the support and the non-uniform interlayer spacing of the pristine $\mathrm{MoS}_{2}$ membranes (,top) translates into nonuniform diffusion of the reagents such as iodomethane. This results in non-uniform functionalization (,bottom) as confirmed by the assymetric broadening of the (002) peak $(\mathrm{a}, \mathrm{b})$. On the other hand, the XPS analyses of the membranes revealed that the average functionalization degree of the membrane is constant (XPS measurements are averaged over a probed size of $400 \mu \mathrm{m}$ in diameter). Therefore, because of $i$ ) the non-uniform 
functionalization observed from the (002) peak and ii) the stable average functionalization degree of the membranes measured from the XPS analyses, we can conclude that for the regions of the membranes where the reagents can easily diffuse in, the extent of methyl groups is larger than the average value measured from the XPS analyses. At the opposite, for the other regions where the reagents have limited access due to steric hindrance, the extent of functional groups is smaller than the average ().

Since the functionalization degree remains constant even at larger thicknesses (Supplementary Figure 19b), we anticipate that as the thickness of the nanolaminate membranes increases, the non-uniform diffusion of the reagent increases and leads to more non-uniform grafting of the methyl groups. Our numerical simulations (See "Concentration of the functional groups" section for more details) have however predicted that the larger the amount of methyl on $\mathrm{MoS}_{2}$, the larger the water flux. The unexpected water flux for thick $\mathrm{MoS}_{2}$-Met. membranes is attributed to non-uniform functionalization degree and locally larger density of methyl groups on the nanosheets.

Thin membranes

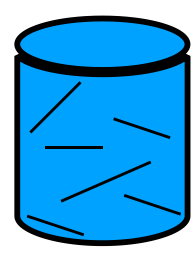

Thick membranes

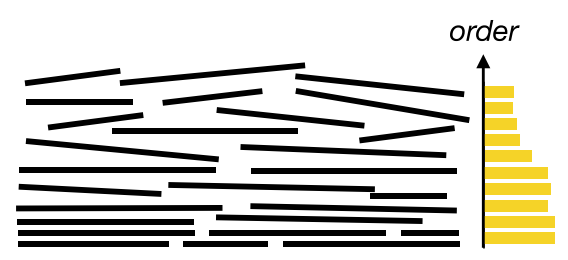




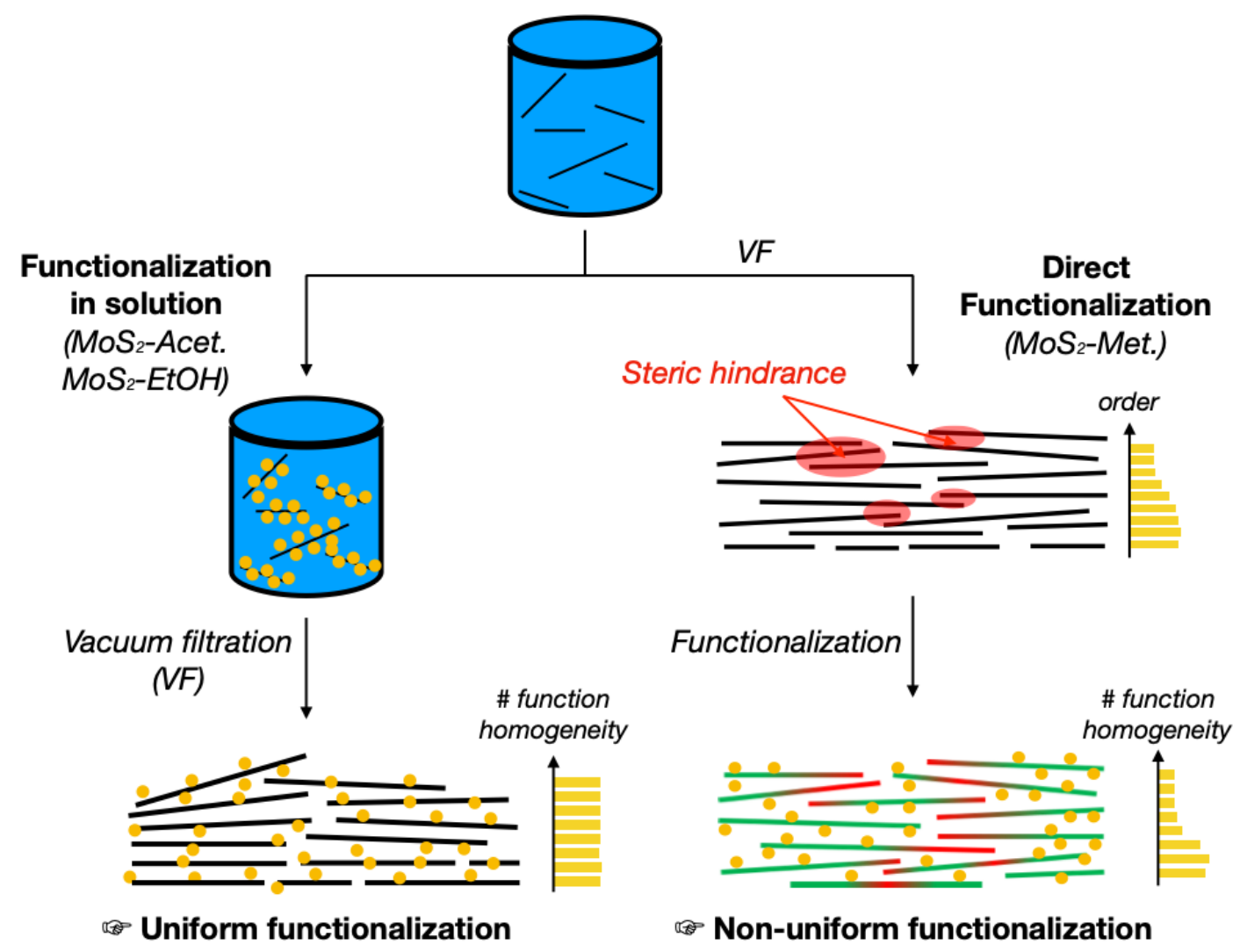

Supplementary Figure 30. Top: Representation of the turbostatic stacking of the nanosheets in the nanolaminate membranes prepared via vacuum filtration (VF). Bottom: Influence of the membrane fabrication on the functionalization homogeneity. In the case of $\mathrm{MoS}_{2}$-Acet. and $\mathrm{MoS}_{2}-\mathrm{EtOH}$, the functionalization of the nanosheets is uniform because the nanosheets are functionalized in solution. In the case of the direct functionalization with iodomethane, the lower interlayer spacing limits the diffusion of the reagents due to steric hindrance. This translates into locally lower functionalization (in red) while the other regions are more decorated with methyl groups (in green).

Our results show that the nanosheets are slightly more functionalized on the top surface compared to the core of the membrane and the functionalization degree per $\mathrm{S}$ atom varies from $25 \%$ down to $19 \%$ for the top surface and the $2 \mu \mathrm{m}$-thick membrane respectively. Importantly our measurements also reveal that the amount of grafted 
functional groups on the $\mathrm{MoS}_{2}$ nanosheets in the membrane remains virtually constant for thicknesses as high as $2 \mu \mathrm{m}$. On the other hand, the full width at half maximum (FWHM) of the (002) XRD peak increases for all the membranes as the thickness increases. The FWHM of the (002) peak for the $\mathrm{MoS}_{2}$-Met. membranes however deviates from the evolution of the (002) peak of $\mathrm{MoS}_{2}$-Acet. membranes for thicknesses larger than $1 \mu \mathrm{m}$ suggesting further disorder in the case of methyl-functionalized $\mathrm{MoS}_{2}$ nanosheets (Supplementary Figs. 20,21).

\section{Velocity profile and slip length measurements}

We calculated the slip length for pristine $\mathrm{MoS}_{2}$ and methyl-functionalized $\mathrm{MoS}_{2}$. The slip length was obtained by fitting the velocity profile of water molecules inside wide $2 \mathrm{D}$ channels of approximately $40 \AA$ separation following the method proposed by Wang et al. $^{21}$. The velocity profiles and corresponding fits for pristine and methyl-MoS 2 are shown in Supplementary Figure 31. Our MD results show that the slip length $L_{S}$ is $-1.70 \AA$ in the case of pristine $\mathrm{MoS}_{2}$ nanosheets. A negative slip length is expected in the case of hydrophilic membranes. For example, negative slip length was predicted between charged graphene membranes and water in the aforementioned reference. The authors found that the values for $L_{S}$ can be tuned from $78 \AA$ for pristine graphene to $-1.07 \AA$ when partial charges are introduced. Since $1 \mathrm{~T}^{\prime} \mathrm{MoS}_{2}$ membranes are rather hydrophilic, our values of $\mathrm{L}_{\mathrm{S}}$ are consistent with the results from Wang et al. In the case of methylfunctionalized $\mathrm{MoS}_{2}$ membranes, $\mathrm{L}_{\mathrm{S}}$ increases to $-1.38 \AA$, consistent with the decrease of the hydrophilicity of the $\mathrm{MoS}_{2}$ slabs. As discussed in the Molecular Dynamics section, the reactive force field overestimates surface tension at the $\mathrm{MoS}_{2}$ /water interface and 
therefore leads to more hydrophilic $\mathrm{MoS}_{2}$. However, this does not affect the comparative nature of the analysis between functional groups. Our simulations predict that the presence of methyl functional groups lead to improved slip length and velocity profiles due to less hydrophilic surface.

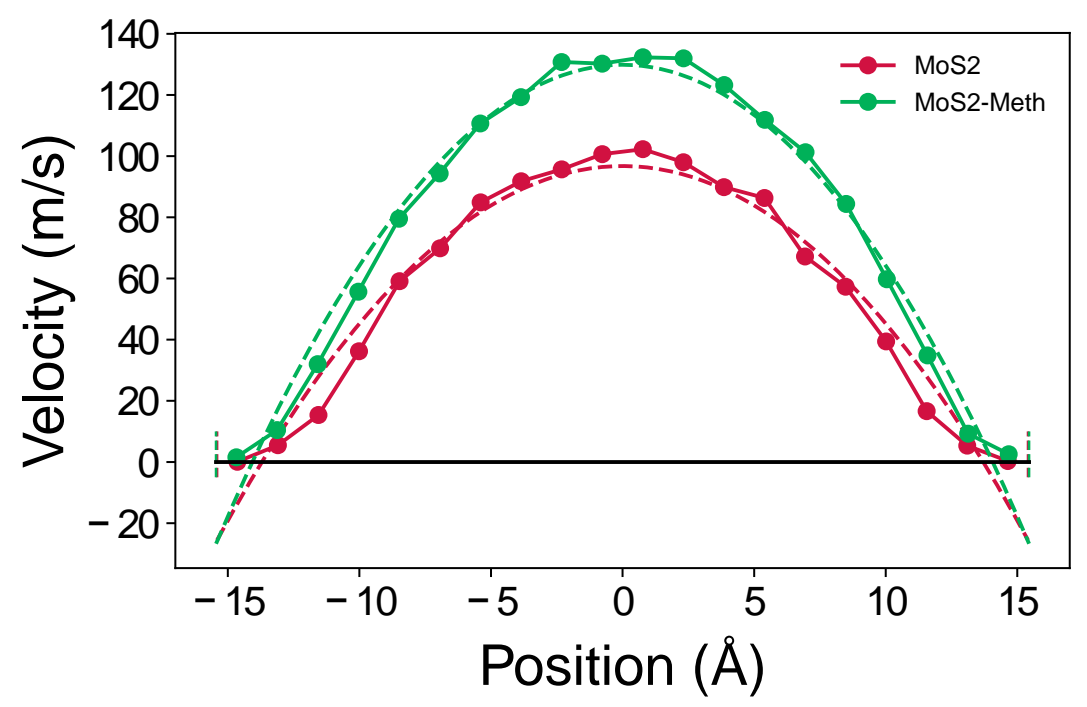

Supplementary Figure 31. Velocity profiles of water in pristine (red) and methylfunctionalized $\mathrm{MoS}_{2}$ channels.

\section{Molecular sieving performances under reversed osmosis conditions}

To evaluate the sieving properties of membranes towards nanocontaminants, the $\mathrm{MoS}_{2}$ membranes were tested under reverse osmosis (RO) conditions as described above. The upstream reservoir was filled with aqueous solution of caffeine, acetaminophen, estradiol $\beta$, or Ni-phtalocyanine (Nickel(II) phthalocyanine-tetrasulfonic acid tetrasodium salt, $\mathrm{NiPc}$ ) (respectively with concentrations of $0.05 \mathrm{M}, 0.5 \mathrm{M}, 11 \mu \mathrm{M}$ and $0.33 \mathrm{mM}$ ). Experiments have been performed at 2 bars loading for every contaminant solutions. The 
permeate solutions were collected and analyzed using high performance liquid chromatography coupled UV-vis detector (HPLC/UV/Fluo). HPLC characterization has been recovered using an APEX WP ODS column $(7 \mu 25 \mathrm{~cm} / 4.6 \mathrm{~mm})$ and data analysis were performed with EMPOWER build 1154 software.

Physico-chemical properties of the contaminant tested are reported in Supplementary Table 7. The Stoke radius - that represents the effective hydrated size of molecules in water - has been calculated using Equation (4).

$$
r=\frac{k_{B} \times T}{6 \pi \times \eta \times D} \quad(4)
$$

where $\mathrm{k}_{\mathrm{B}}$ is Boltzmann's constant $\left(1.38 \times 10^{-23} \mathrm{~J} \mathrm{~K}^{-1}\right), \mathrm{T}$ is the temperature $(\mathrm{K}), \eta$ the kinematic viscosity of the solvent $\left(\mathrm{kg} \mathrm{ms}^{-1}\right)$ and $\mathrm{D}$ is the diffusion coefficient of the contaminants $\left(\mathrm{m}^{2} \mathrm{~s}^{-1}\right)$.

Supplementary Table 7. Physico-chemical properties of contaminants. The Stoke radii were calculated at $25{ }^{\circ} \mathrm{C}$. $*$ Stoke radius of NiPc has been estimated based on the Stoke radius of porphyrin ${ }^{22}$.

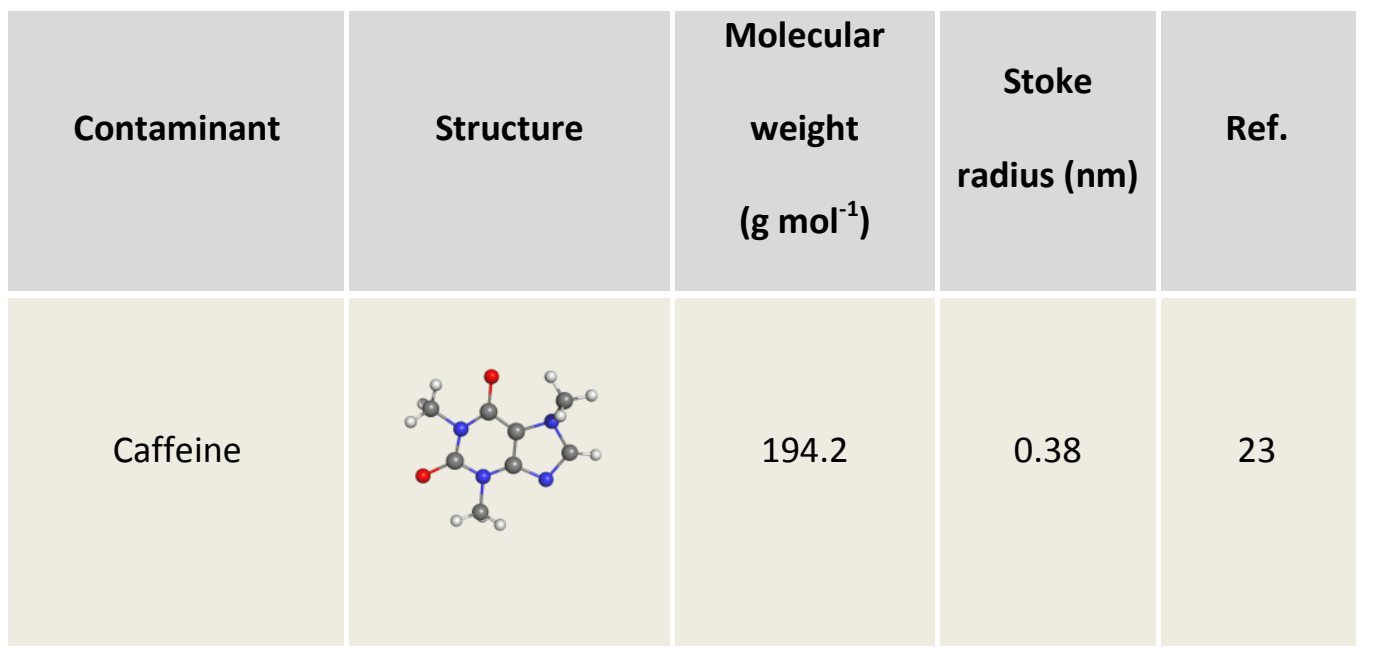




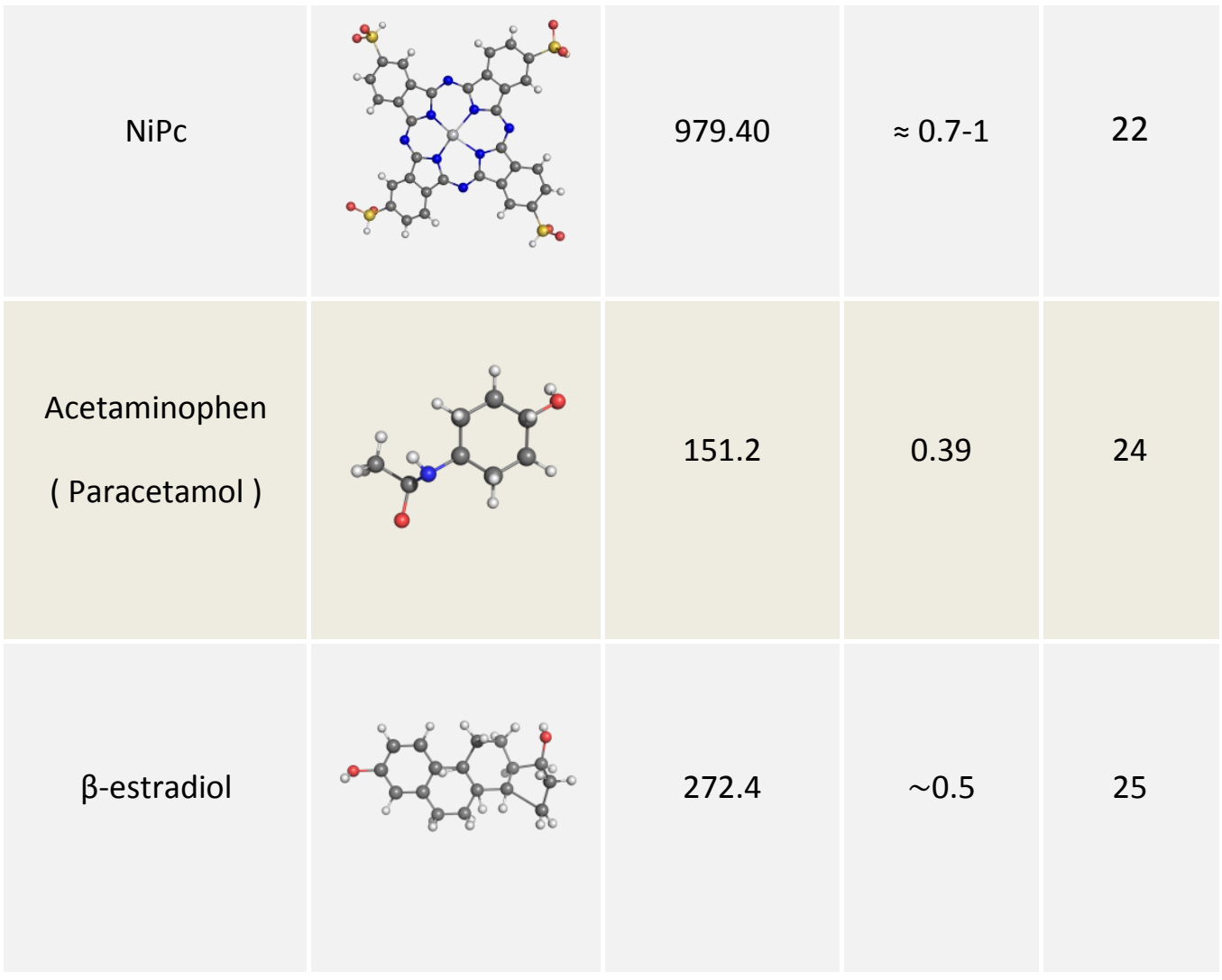

\section{Desalination performance under reverse osmosis conditions}

The desalination performance was evaluated using the same methodology as for the nanocontaminants with a feed concentration of $\mathrm{NaCl}$ of $0.1 \mathrm{M}(0.58$ wt. \%). The concentration of $\mathrm{NaCl}$ in the permeate was measured using Inductively Coupled Plasma Optical Emission Spectrometry (ICP-OES). ICP OES measurements were obtained using an ICAP 7400 ICP-OES Analyzer spectrometer (THERMO SCIENTIFIC). Supplementary Figure 32 summarizes the desalination performance of the various functionalized $\mathrm{MoS}_{2}$ membranes under reverse osmosis configuration. Desalination peaks at $95 \% \mathrm{NaCl}$ rejection for methyl-functionalized membranes. 


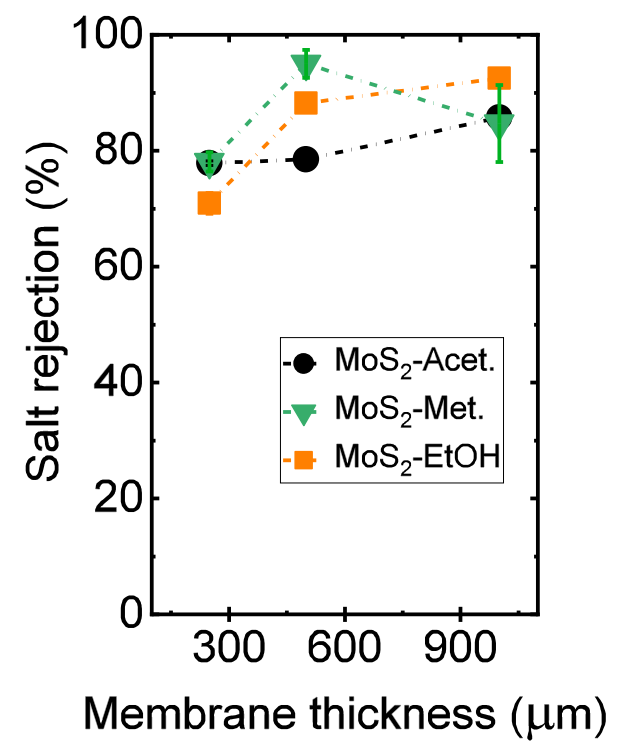

Supplementary Figure 32. $\mathrm{NaCl}$ rejection for the different functionalized $\mathrm{MoS}_{2}$ membranes with thicknesses of $250 \mathrm{~nm}, 500 \mathrm{~nm}$ and $1 \mu \mathrm{m}$.

Under the same conditions $(0.1 \mathrm{M} \mathrm{NaCl})$, we tested the functionalized $\mathrm{MoS}_{2}$ membranes towards desalination under reverse osmosis at an applied pressure of 9 bar. The rejection performances are largely maintained even at 9 bar (Supplementary Figure 33 Supplementary Figure 26 and Supplementary Table 8). We note that the values of water flux at 9 bar differ from those measured at lower pressures up to 4 bar due to differences in stiffness of the functionalized nanolaminate membranes. 


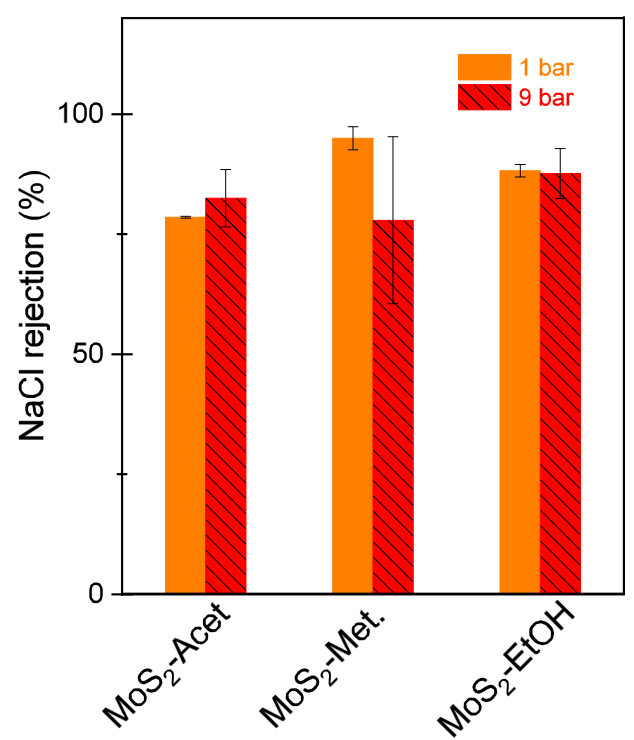

Supplementary Figure 33. Comparison of the desalination performance at 1 and 9 bar obtained for $500 \mathrm{~nm}$-thick functionalized $\mathrm{MoS}_{2}$ membrane.

Supplementary Table 8. Rejection performance of the $500 \mathrm{~nm}$-thick functionalized $\mathrm{MoS}_{2}$ membranes under a pressure of $9 \mathrm{bar}$.

\begin{tabular}{c|c|c|}
\hline Samples & $\begin{array}{c}\text { NaCl Rejection at } \\
\mathbf{1} \text { bar }(\%)\end{array}$ & $\begin{array}{c}\text { NaCl Rejection at } \\
\text { 9 bar }(\%)\end{array}$ \\
\hline MoS $_{2}$-Acet. & $78.5( \pm 0.2)$ & $82.5( \pm 6.0)$ \\
\hline MoS $_{2}$-Met. & $95.0( \pm 2.4)$ & $77.9( \pm 17.4)$ \\
\hline MoS $_{2}$-EtOH & $88.2( \pm 1.3)$ & $87.6( \pm 5.2)$ \\
\hline
\end{tabular}

Comparison of the performance of the $\mathrm{MoS}_{2}$ membranes with the state of the arts

The desalination performances have been compared with the literature (main Figure 3D) and the corresponding data are detailed in Supplementary Table 9. 
Supplementary Table 9. Comparison of the performances of functionalized $\mathrm{MoS}_{2}$ membranes with performance from the state of the arts. RO: reverse osmosis; SWRO: Sea water Reverse Osmosis; BWRO: Brackish Water Reverse Osmosis; NF: Nanofiltration; NR: not reported; TFC: Thin Film Composite; CNT: Carbon Nanotube, GO: Graphene oxide.

\begin{tabular}{|c|c|c|c|c|c|c|c|}
\hline Materials & $\begin{array}{c}\text { Filtration } \\
\text { method }\end{array}$ & $\begin{array}{c}\text { Thickness } \\
(\mathbf{n m})\end{array}$ & $\begin{array}{r}\text { Press. } \\
\text { (bar) }\end{array}$ & $\begin{array}{c}\mathrm{NaCl} \text { feed } \\
{[](\mathrm{M})}\end{array}$ & $\begin{array}{c}\text { Water } \\
\text { flux } \\
\text { L/(h.m².bar) }\end{array}$ & $\begin{array}{c}\mathrm{NaCl} \\
\text { rejection }\end{array}$ & Ref. \\
\hline $\begin{array}{c}\text { Non } \\
\text { functionalized } \\
\mathrm{MoS}_{2}\end{array}$ & Dead end & 250-1000 & $0.5-4$ & 0.1 & $\begin{array}{c}\mathbf{0} \\
\text { (pure water) }\end{array}$ & - & This work \\
\hline $\begin{array}{l}\text { Acetamide- } \\
\text { functionalized } \\
\qquad \mathrm{MoS}_{2}\end{array}$ & $\begin{array}{c}\text { Dead end } \\
\text { FO }\end{array}$ & \begin{tabular}{r|}
250 \\
500 \\
1000 \\
1000 \\
250
\end{tabular} & 1 & 0.1 & $\begin{array}{c}13.7( \pm 5.0) \\
(\text { pure water }) \\
9.3( \pm 2.8) \\
(\text { pure water }) \\
3.1( \pm 1.6) \\
\text { (pure water }) \\
0.036( \pm 0.006) \\
0.052( \pm 0.005)\end{array}$ & $\begin{array}{l}77.9( \pm 0.3) \\
78.5( \pm 0.2) \\
85.7( \pm 0.2)\end{array}$ & This work \\
\hline $\begin{array}{l}\text { Acetamide- } \\
\text { functionalized } \\
\qquad \mathrm{MoS}_{2}\end{array}$ & Dead end & 500 & 9 & 0.1 & $33.7( \pm 13.5)$ & $82.5( \pm 6)$ & This work \\
\hline $\begin{array}{l}\text { Ethyl-2-ol- } \\
\text { functionalized } \\
\mathrm{MoS}_{2}\end{array}$ & Dead end & $\begin{array}{l}250 \\
500\end{array}$ & 1 & 0.1 & $\begin{array}{c}42.5( \pm 12.1) \\
(\text { pure water }) \\
13.4( \pm 3.0) \\
(\text { pure water }) \\
7.8( \pm 5.0) \\
(\text { pure water }) \\
2.1( \pm 0.4) \\
\text { (pure water) }\end{array}$ & $\begin{array}{l}70.9( \pm 1.8) \\
88.2( \pm 1.2) \\
85.7( \pm 1.0)\end{array}$ & This work \\
\hline
\end{tabular}




$\begin{array}{ccc} & 1000 & 0.092( \pm 0.02) \\ \text { FO } & 250 & 0.146( \pm 0.04)\end{array}$

Ethyl-2-ol-

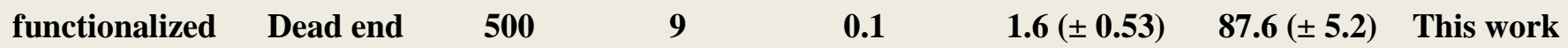
$\mathrm{MoS}_{2}$

Methyl-

$\begin{array}{ccc}\text { functionalized } & \text { Dead end } & 500 \\ \mathrm{MoS}_{2} & & 1000 \\ & & 2000\end{array}$

Methyl-
250

500

000

2000

$$
2.9( \pm 1.6)
$$

(pure water) $78.1( \pm 1.6)$

$15.9( \pm 4.0) \quad 95.0( \pm 2.4)$

0.1

(pure water)

9.8 ( \pm 5.2$)$

(pure water)

24.8 ( \pm 0.6$)$

(pure water)

\begin{tabular}{|c|c|c|c|c|c|c|c|}
\hline $\begin{array}{c}\text { functionalized } \\
\qquad \mathrm{MoS}_{2}\end{array}$ & Dead end & 500 & 9 & 0.1 & $6.7( \pm 1.35)$ & $77.9( \pm 17.4)$ & This work \\
\hline $\begin{array}{c}\text { Higly laminated } \\
\text { GO }\end{array}$ & Dead end & 8 & 2 & 1 & 4 & 25 & 26 \\
\hline $\begin{array}{c}\text { Higly laminated } \\
\text { GO }\end{array}$ & Dead end & 150 & 2 & & 0.3 & & 26 \\
\hline $\mathrm{MoS}_{2}$ & Dead end & 1700 & 1 & & 245 & NR & 27 \\
\hline GO/TMC & Dead end & 14 & 3.4 & 0.02 & $\approx 50$ & 19 & 28 \\
\hline Graphene & Dead end & $260-330$ & 1 & 0.02 & 20 & 40 & 14 \\
\hline GO/CNT & Dead end & $<100$ & 5 & 0.01 & 5.5 & 59 & 29 \\
\hline Graphene/CNT & Dead end & $\approx 40$ & 5 & 0.01 & 11.3 & 51 & 29 \\
\hline GO/ PECs & Cross flow & - & 5 & - & 0.7992 & 43 & 30 \\
\hline Graphene/CNT & Cross flow & - & 5 & 0.01 & 12.13 & 39.6 & 29 \\
\hline Graphene/GO & Cross flow & $260-330$ & 50 & 0.04 & $0.34( \pm 0.1)$ & $85( \pm 2)$ & 31 \\
\hline
\end{tabular}




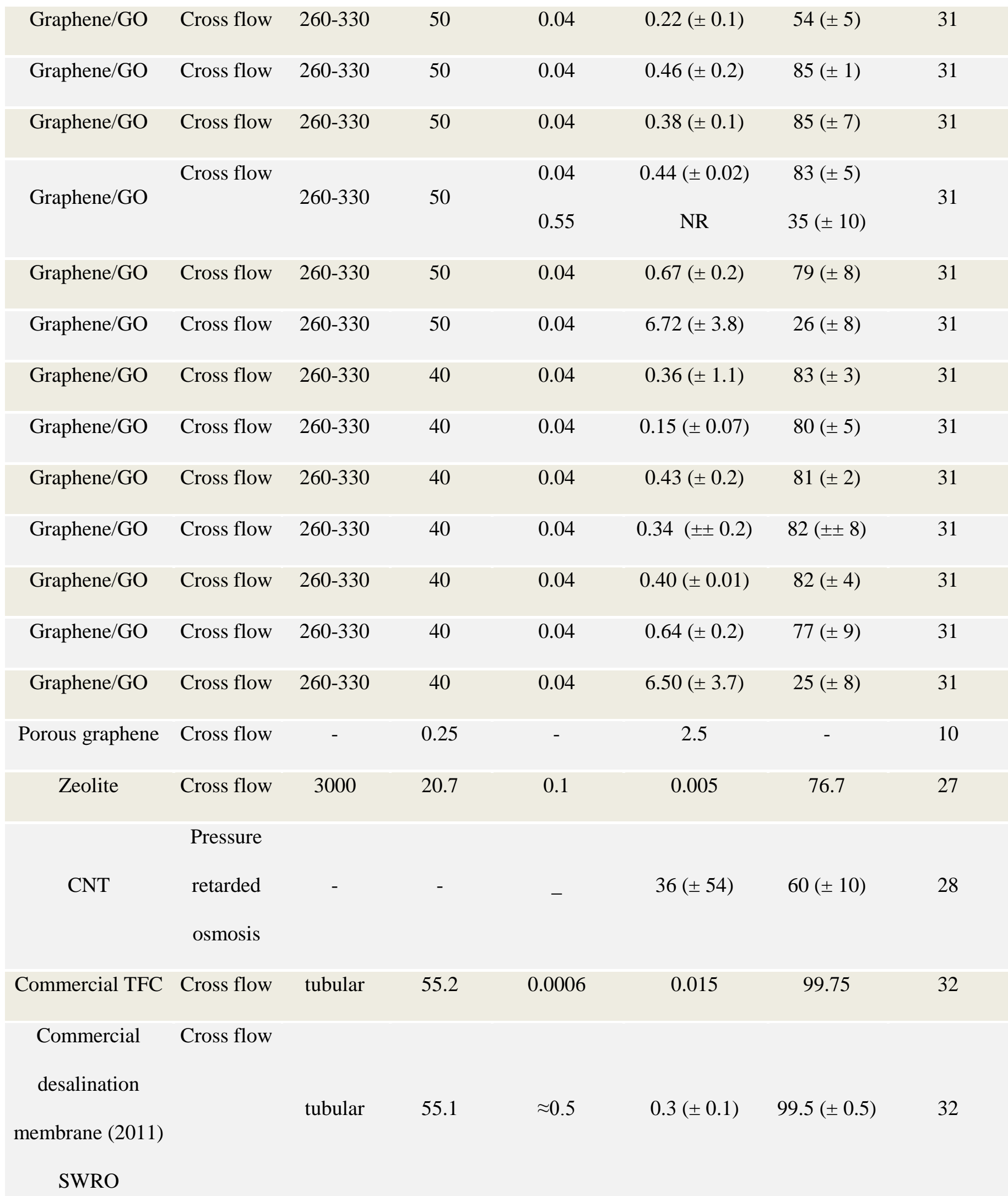




\begin{tabular}{|c|c|c|c|c|c|c|c|}
\hline BWRO & Cross flow & tubular & $6.9-41.4$ & $\begin{array}{c}0.0086- \\
0.034\end{array}$ & $2( \pm 2)$ & $94( \pm 1.425)$ & 32 \\
\hline NF & Cross flow & tubular & $5.2-15.9$ & $\begin{array}{c}0.0086- \\
0.034\end{array}$ & $5.5( \pm 1)$ & $65( \pm 15)$ & 32 \\
\hline $\begin{array}{c}\text { Commercial TFC } \\
\text { (2016) }\end{array}$ & Cross flow & $\begin{array}{l}\text { Spiral } \\
\text { wound }\end{array}$ & 55 & $\approx 0.5$ & $1.19( \pm 0.24)$ & 99.7 & 33 \\
\hline $\begin{array}{l}\text { Commercial TFC } \\
\text { (US, 2017) }\end{array}$ & Cross flow & $\begin{array}{l}\text { Spiral } \\
\text { wound }\end{array}$ & 56 & $\approx 0.5$ & $0.68( \pm 0.14)$ & 99.8 & 34 \\
\hline $\begin{array}{l}\text { Commercial TFC } \\
\text { polyamide }\end{array}$ & Cross flow & $\begin{array}{l}\text { spiral } \\
\text { wound }\end{array}$ & 15.5 & $\approx 0.55$ & 2.8 & 99.5 & 35 \\
\hline $\begin{array}{c}\text { Commercial TFC } \\
\text { polyamide }\end{array}$ & Cross flow & $\begin{array}{l}\text { spiral } \\
\text { wound }\end{array}$ & 55 & $\approx 0.55$ & 0.47 & 99.7 & 36 \\
\hline $\begin{array}{c}\text { Commercial TFC } \\
\text { polyamide }\end{array}$ & Cross flow & $\begin{array}{l}\text { spiral } \\
\text { wound }\end{array}$ & 55 & $\approx 0.55$ & 0.85 & 99.7 & 37 \\
\hline $\begin{array}{c}\text { Commercial TFC } \\
\text { polyamide }\end{array}$ & Cross flow & $\begin{array}{l}\text { spiral } \\
\text { wound }\end{array}$ & 55 & $\approx 0.55$ & 0.7 & 99.5 & 38 \\
\hline $\begin{array}{c}\text { Commercial TFC } \\
\text { polyamide }\end{array}$ & Cross flow & $\begin{array}{l}\text { spiral } \\
\text { wound }\end{array}$ & 55 & $\approx 0.55$ & 0.57 & 99.7 & 39 \\
\hline $\begin{array}{l}\text { Commercial TFC } \\
\text { polyamide }\end{array}$ & Cross flow & $\begin{array}{l}\text { spiral } \\
\text { wound }\end{array}$ & 55 & $\approx 0.55$ & 0,49 & 99.7 & 40 \\
\hline $\begin{array}{c}\text { Commercial TFC } \\
\text { polyamide }\end{array}$ & Cross flow & $\begin{array}{l}\text { spiral } \\
\text { wound }\end{array}$ & 55 & $\approx 0.55$ & 0,62 & 99.7 & 41 \\
\hline $\begin{array}{c}\text { Commercial TFC } \\
\text { polyamide }\end{array}$ & Cross flow & $\begin{array}{l}\text { spiral } \\
\text { wound }\end{array}$ & 55 & $\approx 0.55$ & 0,63 & 99.7 & 42 \\
\hline
\end{tabular}




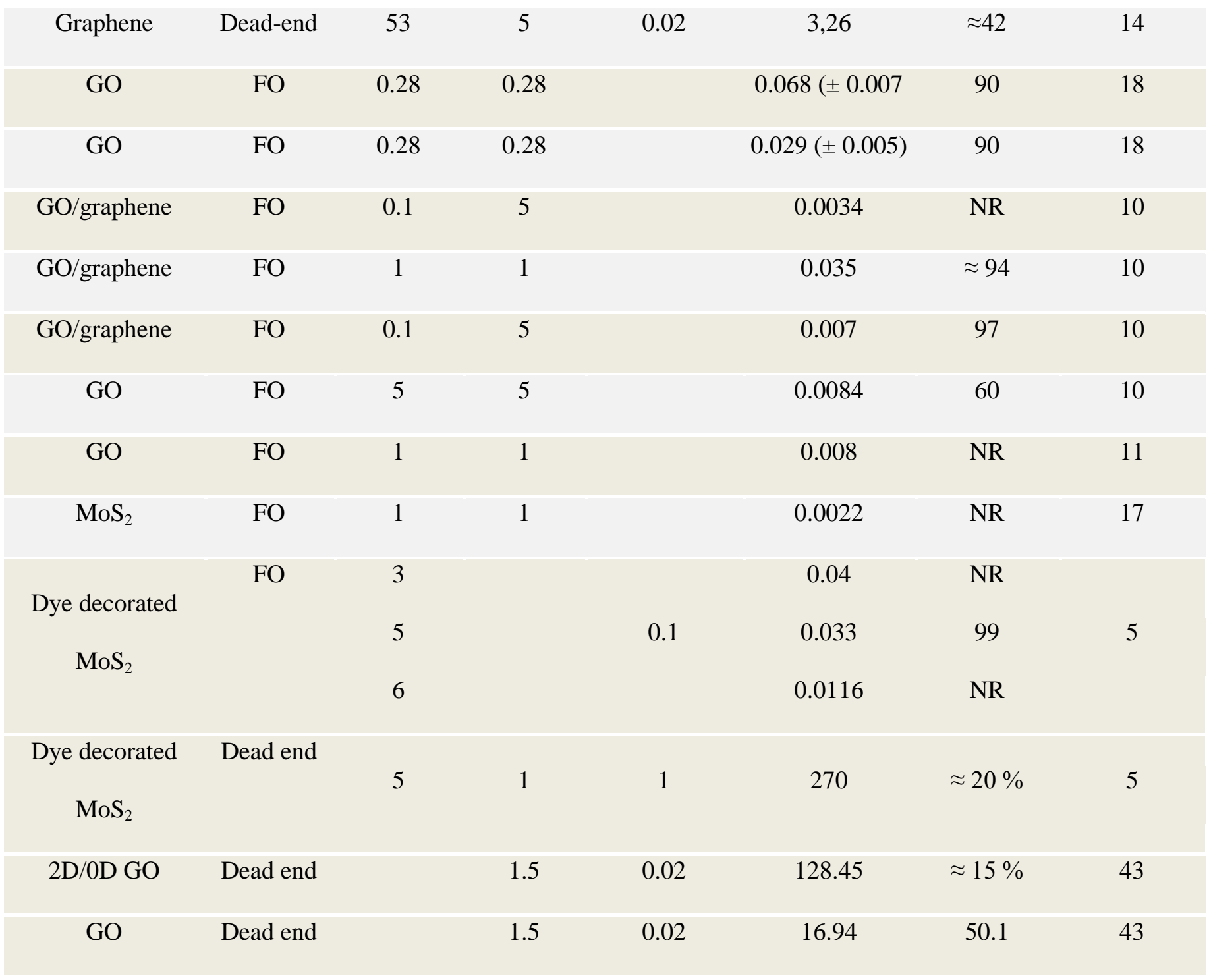




\section{Molecular dynamics simulations}

\section{Reactive MD and interatomic potential}

We performed fully reactive molecular dynamics simulations with $\operatorname{ReaxFF}^{45}$. C, H, O and $\mathrm{N}$ force field parameters were taken from Ref. ${ }^{45}$ and combined with that of $\mathrm{MoS}_{2}$ from Ref. ${ }^{46}$. We slightly modified the S-O van der Waals (vdW) parameters to improve the water/ $\mathrm{MoS}_{2}$ interactions. These parameters have been optimized against density functional theory (DFT) calculations corresponding to the adsorption of a single water molecule on a monolayer $\mathrm{MoS}_{2}$ and, water molecules in between $\mathrm{MoS}_{2}$ layers. Supplementary Figure 34 shows the corresponding potential energy surfaces before and after optimization of the parameters, summarized in Supplementary Table 10. We note that this adjustment only affects the water/MoS 2 interactions since sulfur is not part of any functional group studied. Second order interactions such as Mo with $\mathrm{C}, \mathrm{H}, \mathrm{O}$ and $\mathrm{N}$ were taken from Ref. $^{47}$.
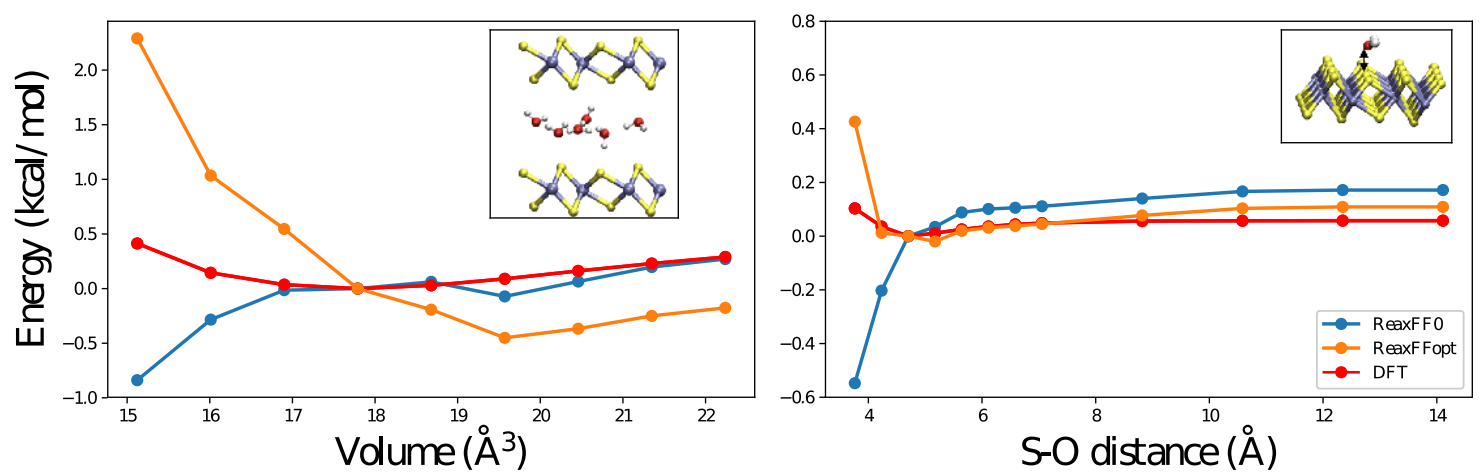

Supplementary Figure 34. ReaxFF optimization. ReaxFF was optimized to describe the energy of bulk $\mathrm{MoS}_{2}$ intercalated with 6 water molecules as a function of the out of plane lattice parameter (left) and, the adsorption of a water molecule on the surface of $\mathrm{MoS}_{2}$ as a function of S-O distance (right). 
Supplementary Table 10. Original and optimized ReaxFF off-diagonal parameters for S-O interaction.

\begin{tabular}{|c|c|c|c|c|}
\hline & Ediss & Ro & Gamma & rsigma \\
\hline $\begin{array}{c}\text { Original from } \\
\text { Ref. 45 }\end{array}$ & 0.1962 & 1.7872 & 10.2319 & 1.4622 \\
\hline $\begin{array}{c}\text { Optimized } \\
\text { parameters }\end{array}$ & 0.2132 & 2.2373 & 11.3611 & 1.1245 \\
\hline
\end{tabular}

We acknowledge that our reactive force field does not lead to stable water drop on a perfect $\mathrm{MoS}_{2}$ surface, as it is often used to validate the water/surface interaction ${ }^{48}$. Although there is no proof that the adhesion of a water drop on surface and its confinement in sub-nanometer channels behave similarly ${ }^{49}$. We found that the water drop completely wet the $\mathrm{MoS}_{2}$ surface after approximately $1 \mathrm{~ns}$ of equilibration. Therefore, our model overestimates surface tension at the water/MoS ${ }_{2}$ interface and as a consequence, underestimates the absolute water flux. However, the relative flux between different $\mathrm{MoS}_{2}$ functionalized surfaces and various interlayer spacing discussed here are relevant. Moreover, we want to stress that the proposed reactive force field is the only model available to describe with precision such complex surfaces including partial charges and polarization effects including that of the lone pairs on $\mathrm{O}$ and $\mathrm{S}$ elements. Preliminary tests with state-of-the-art water/ $\mathrm{MoS}_{2}$ interatomic potentials led to very high hydrophobicity of the functionalized channels mainly because of the static description of partial charges and the lack of parameters for inorganic/organic surfaces ${ }^{50}$. Future work should focus on the improvement of the reactive force field in order to improve the description of the contact angle of water on $\mathrm{MoS}_{2}$ surface. 
DFT calculations were performed with $\mathrm{VASP}^{51,52}$ with the Perdew, Burke, and Ernzerhof functional $^{53}$ including Grimme's D2 correction to improve vdW interactions ${ }^{54}$. Both monolayer and bulk supercells were built by replicating $4 \times 4$ times the rectangular unit cell of $\mathrm{MoS}_{2}$ in the in-plane directions. A $2 \times 2 \times 1$ and $1 \times 1 \times 2 \mathrm{k}$-grid was used for monolayer and bulk calculations, respectively. We used a $400 \mathrm{eV}$ kinetic energy cutoff and, we assumed convergence when energy and forces reached thresholds of $10^{-4} \mathrm{eV}$ and $5 \times 10^{-2} \mathrm{eV} \AA^{-1}$, respectively.

\section{Nanofluidic simulations}

Nanofluidic transport simulations were performed using the fluctuating wall method. We followed ideas from Berendsen's thermostat ${ }^{55}$ to adjust the position of two harmonic walls (which interact with water via a simple harmonic potential) perpendicular to the channel and located at the boundaries of the simulation cell (main Figure 4a). The position of one wall $x$ at time $t$ is defined as in Equation (5):

$$
\left\{\begin{array}{c}
\mu=1-\beta \frac{\Delta t}{\tau}\left(P-P_{\text {ext }}\right) \\
x=x_{0}+\mu v_{0} t
\end{array}\right.
$$

with $x_{0}, v_{0}$ the initial position and velocity of the wall, $\mu$ a scaling factor computed every 10 steps in the present simulations, $\beta$ the compressibility, $\Delta t$ the time step, $\tau$ a time constant, $P_{\text {ext }}$ the target pressure and $P$ the pressure computed as the total force on the wall divided by its area. $\beta$ and $\tau$ were optimized to reach fast pressure equilibration resulting in the values 0.05 and 10000 , respectively. The external pressure on the feed 
and permeate walls is represented on Supplementary Figure 35. A $500 \mathrm{MPa}$ pressure gradient is achieved by setting the pressure of the feed reservoir to $510 \mathrm{MPa}$ and that of the permeate reservoir to $10 \mathrm{MPa}$. The water flux was computed from the slope of the number of water molecules crossing the mid-point of the nanochannel as a function of simulation time. We converted units from the number of water molecules per nanoseconds (water $\mathrm{ns}^{-1}$ ) to $\mathrm{L} \mathrm{m}^{-2} \mathrm{~h}^{-1} \mathrm{bar}^{-1}$ by normalizing with the area of the channel (lenght $\times$ width) and dividing by the applied pressure of $500 \mathrm{MPa}$. The number of water molecule per liter was calculated assuming a water density of $1 \mathrm{~g} \mathrm{~cm}^{-3}$.

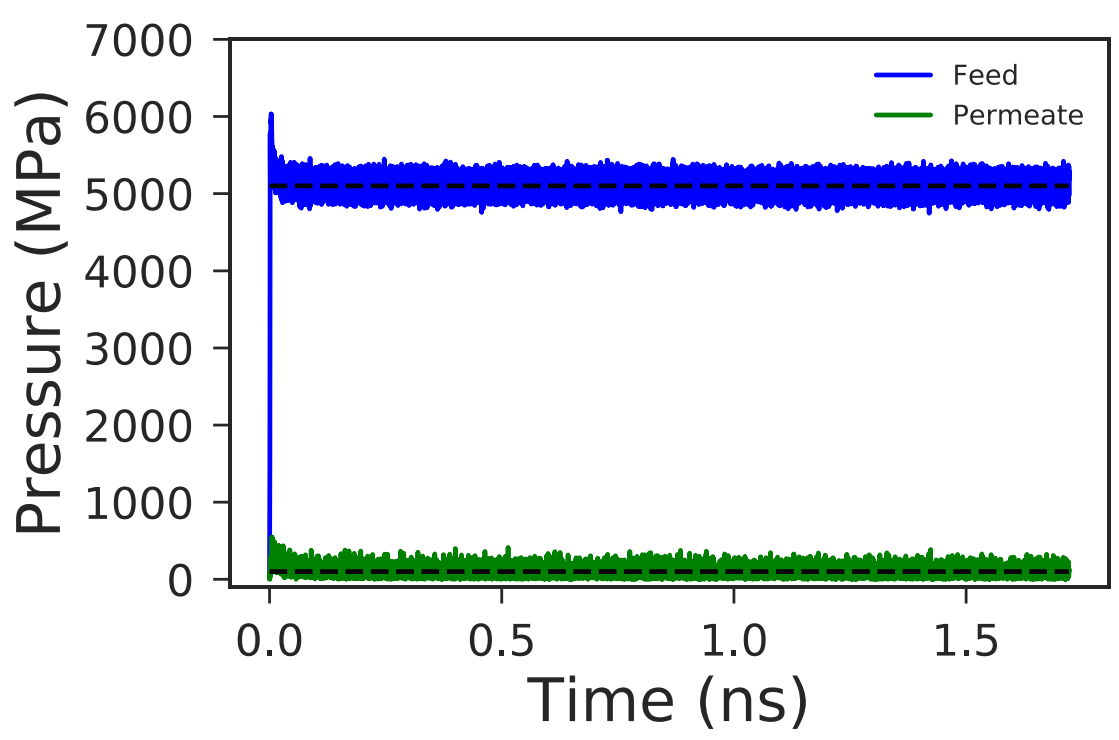

Supplementary Figure 35. Applied pressure on the feed and permeate reservoir using the fluctuating wall method as a function of simulations time. The target pressures are 510 and $10 \mathrm{MPa}$ applied to the feed and permeate reservoirs, respectively. 


\section{Density Profiles}

We performed density profiles of $\mathrm{O}$ and $\mathrm{H}$ atoms along the $\mathrm{y}$-direction (see main Figure $4 \mathrm{a}$ for the reference frame axis) to characterize the structure of water in various nanochannels. Supplementary Figure 36 shows density profiles of pristine $\mathrm{MoS}_{2}$ nanochannels at different capillary widths (corresponding to simulations presented Figure $4 \mathrm{~b}$ in the main text). For pristine $\mathrm{MoS}_{2}$ channels, we found that water molecules are highly organized in layered structure.
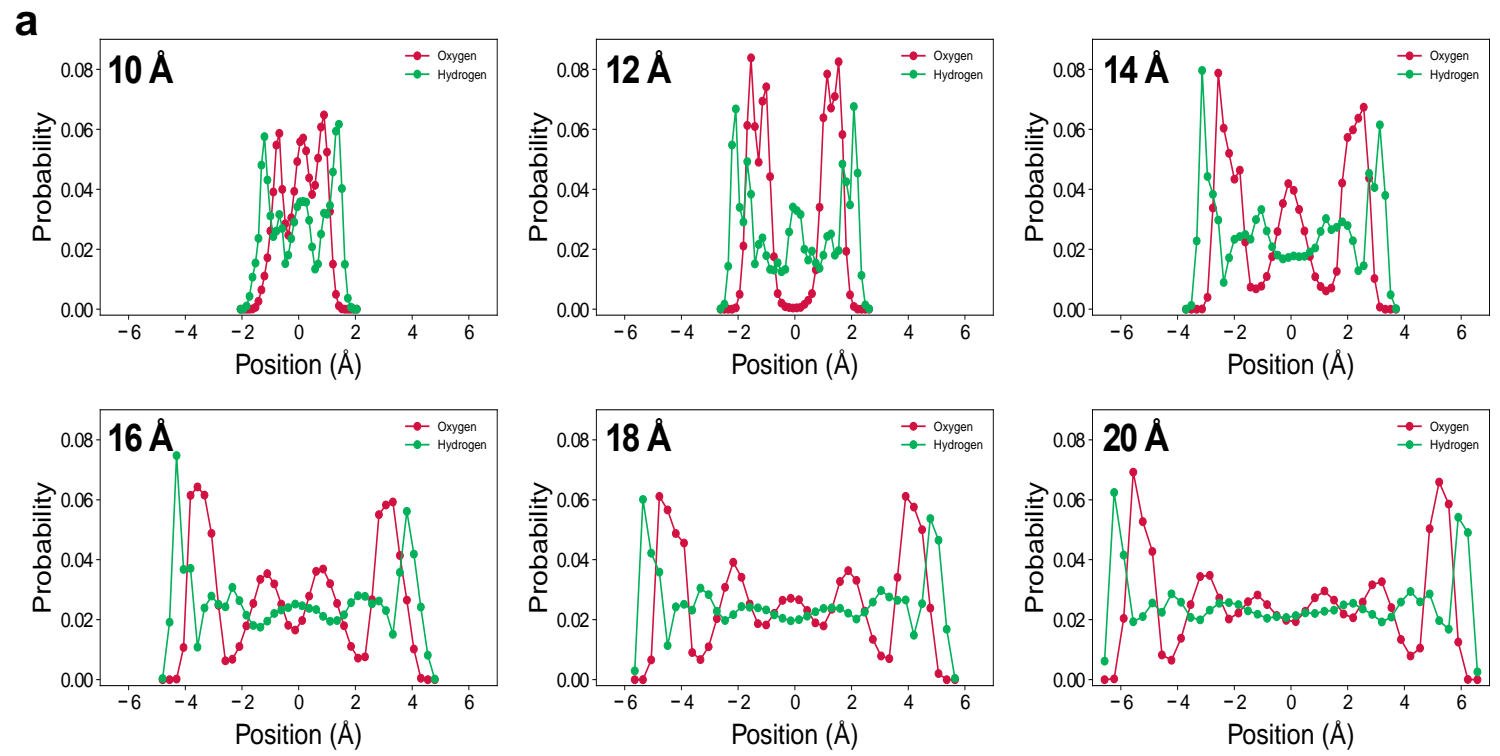

Supplementary Figure 36. Density profiles of $\mathrm{O}$ and $\mathrm{H}$ along the $\mathrm{y}$-direction for pristine $\mathrm{MoS}_{2}$ channels at various capillary widths.

We identified 1, 2, 3, 4, 5 and 6 layers of water in capillaries width of 10, 12, 14, 16, 18 and $20 \AA$ A respectively. This agrees well with recent equilibrium MD simulations ${ }^{56}$ for which the structure of water was associated with the electrostatic interactions between water and $\mathrm{MoS}_{2}$. The introduction of methyl-functionalized channels leads to the 
enlargement of the oxygen peaks at $12 \AA$ separation and, the loss of the 6-layers structure at $20 \AA$ capillary width, compared to pristine channels (Supplementary Figure 37b,d). This shows that water between methyl functionalized channels is less structured compared to water between pristine walls.
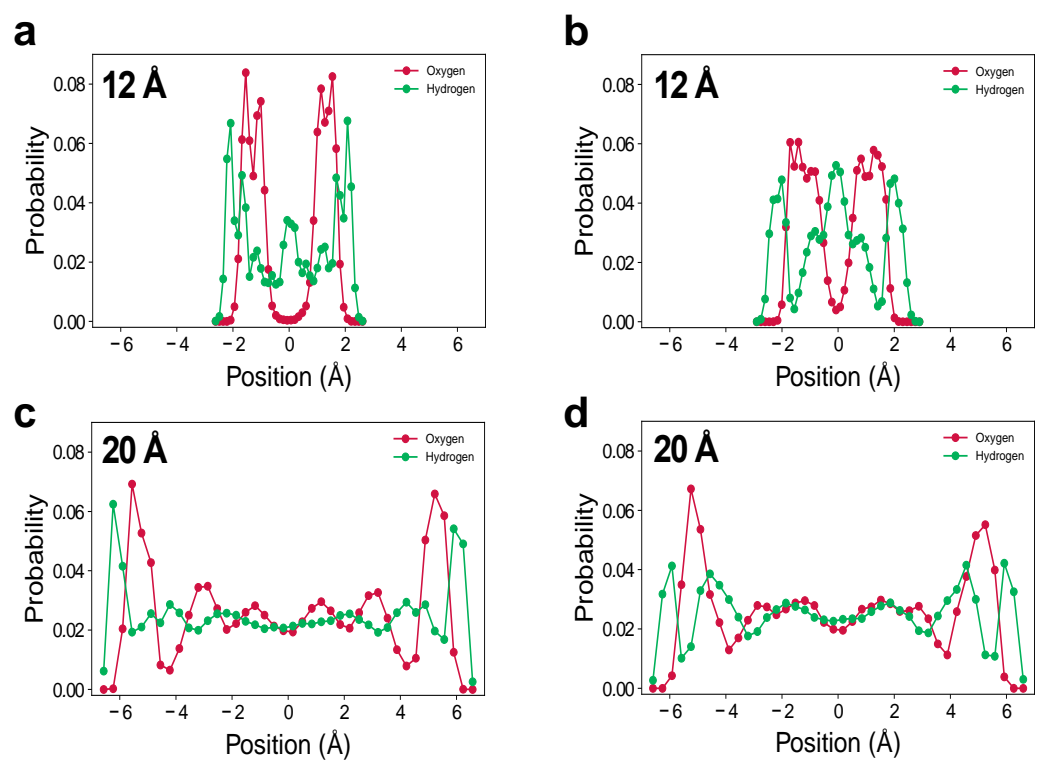

Supplementary Figure 37. Density profiles of $\mathrm{O}$ and $\mathrm{H}$ along the $\mathrm{y}$-direction for pristine (a,c) and methyl-functionalized (b,d) $\mathrm{MoS}_{2}$ channels at various capillary widths.

\section{Axial density function}

The structure of water along the flux direction (x-axis) can be characterized by the axial density function (ADF) ${ }^{49}$. Similar to the radial pair distribution function, the ADF $\mathrm{a}(\mathrm{x})$ represents the probability of finding a water molecule within the interval $\mathrm{x}$ and $\mathrm{x}+\mathrm{dx}$ from a reference water molecule. The ADF is normalized such that its integral over the entire channel equals the total number of water molecules. The ADF for pristine, methyl, acetamide and ethyl-2-ol functionalized channels (corresponding to data in main Figure 
4f) are represented Supplementary Figure 38. Because of the non-periodicity of the channel in the X-direction, the probability of finding a water molecule decreases when $\mathrm{x}$ increases. For pristine case, we can clearly identify the structure of water in the axial direction with a period of approximately $3 \AA$. No clear structure is observed for the functionalized cases.

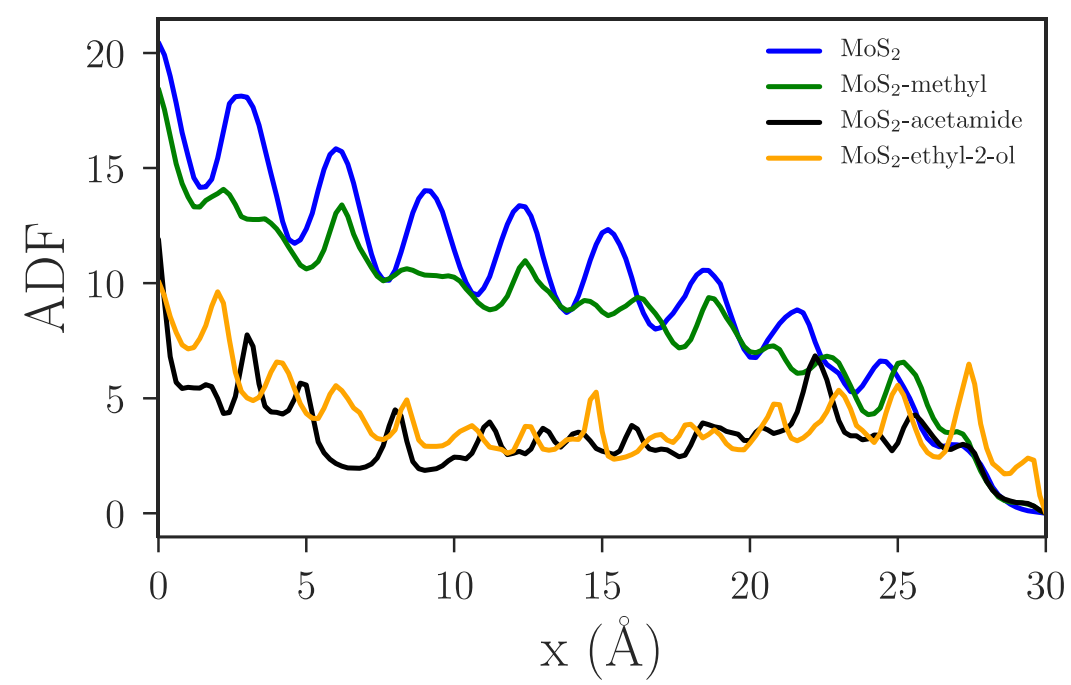

Supplementary Figure 38. Axial density function along the channel direction for various functionalized channels separated by $12 \AA$.

\section{Hydrogen bond correlation function.}

The dynamical properties of water confined can be determined from the hydrogen bond correlation function Hbcorr(t) ${ }^{57}$. We define a hydrogen bond between two water molecules when the distance between their oxygen atoms is less than $3.5 \AA$ and the angle between the vector joining the two oxygen atoms and that corresponding to the O-D bond of the donor water molecule is less than $30^{\circ}{ }^{57}$. The hydrogen bond correlation function is then calculated as $\operatorname{Hbcorr}(\mathrm{t})=\langle\mathrm{h}(0) \mathrm{h}(\mathrm{t})\rangle / \mathrm{h}$ with $\mathrm{h}(\mathrm{t})=1$ when a hydrogen bond exists 
for a particular tagged pair of water molecules and zero otherwise. The hydrogen bond correlation function corresponds to the probability that there is a hydrogen bond at time $t$, given that it was already established at time zero. We show in Supplementary Figure 39 the hydrogen bond correlation function of bulk water and water between various functionalized $\mathrm{MoS}_{2}$ channels, separated by $12 \AA$ (corresponding to data in main Figure 4f). The hydrogen bond lifetime is evaluated from the fit of the curves with the exponential e-t/ $\tau$ and, are reported in the legend of the figure. We found lifetimes of approximately $1 \mathrm{~ns}$ for water inside $12 \AA \mathrm{MoS}_{2}$ capillaries, two orders of magnitude higher than in bulk water. This demonstrates that the interactions between water molecules in confined $\mathrm{MoS}_{2}$ channels are much stronger than in the bulk, characteristic of an ice-like structure and, similar to the arrangement of the water molecules inside carbon nanotubes. The presence of non-polar functional group such as methyl lowers lifetimes by approximately a factor of 2 leading to more bulk-like water.

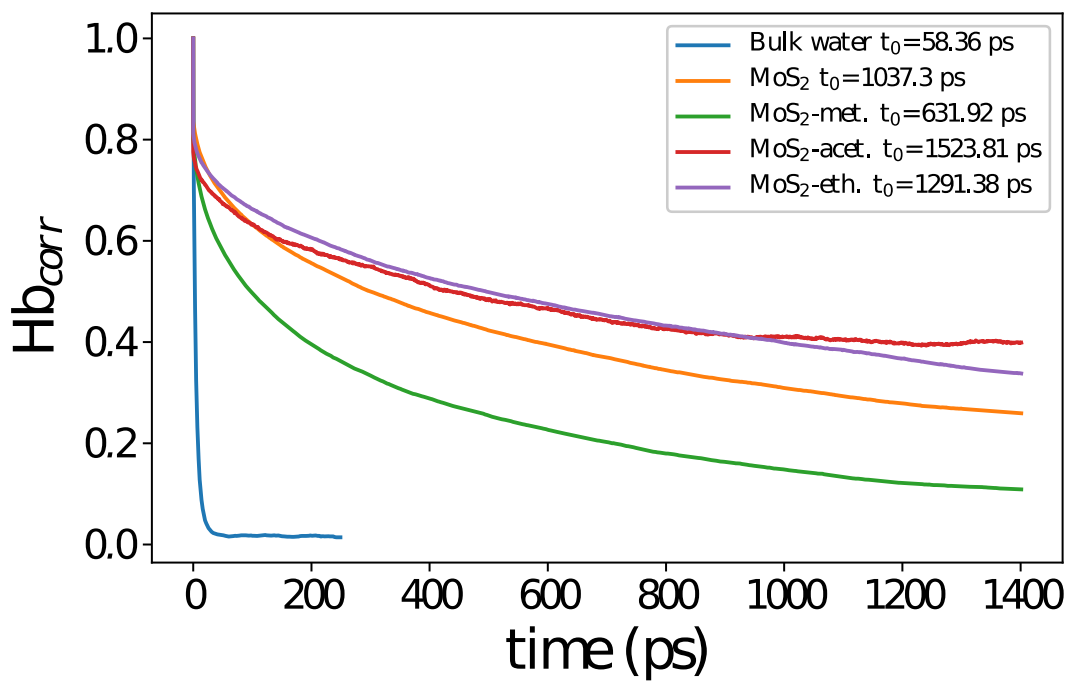

Supplementary Figure 39. Hydrogen bond correlation function of bulk water and water between various functionalized $\mathrm{MoS}_{2}$ channels separated by $12 \AA$. 


\section{Velocity map.}

We found that confined water in $\mathrm{MoS}_{2}$ channels is highly organized with slow hydrogen bond relaxation timescales forming ice-like layers. We now investigate the correlation between the structure of the nanochannel and the dynamical properties of water. More precisely, we would like to see if there is any correlation between the mobility of water in contact with the pristine or functionalized surfaces. We computed the velocity of the first layer of water molecules in contact with pristine and methylfunctionalized $\mathrm{MoS}_{2}$. Supplementary Figure 40 shows the representations of the methyl$\mathrm{MoS}_{2}$ channel side by side including the time averaged velocity map of water (as in main Figure 4e), the same map with in addition all positions of $\mathrm{C}$ and $\mathrm{H}$ atoms of methyl functional groups during the time averaged, and the actual molecular model of the layer of the channel showing all positions of the methyl atoms during the time average. By comparing the 3-graphs, one can conclude that the large majority of methyl atoms' location corresponds to higher averaged velocity of the water molecules underneath. Red (high velocity of water) patches are completely covered by the positions of methyl's atoms. 

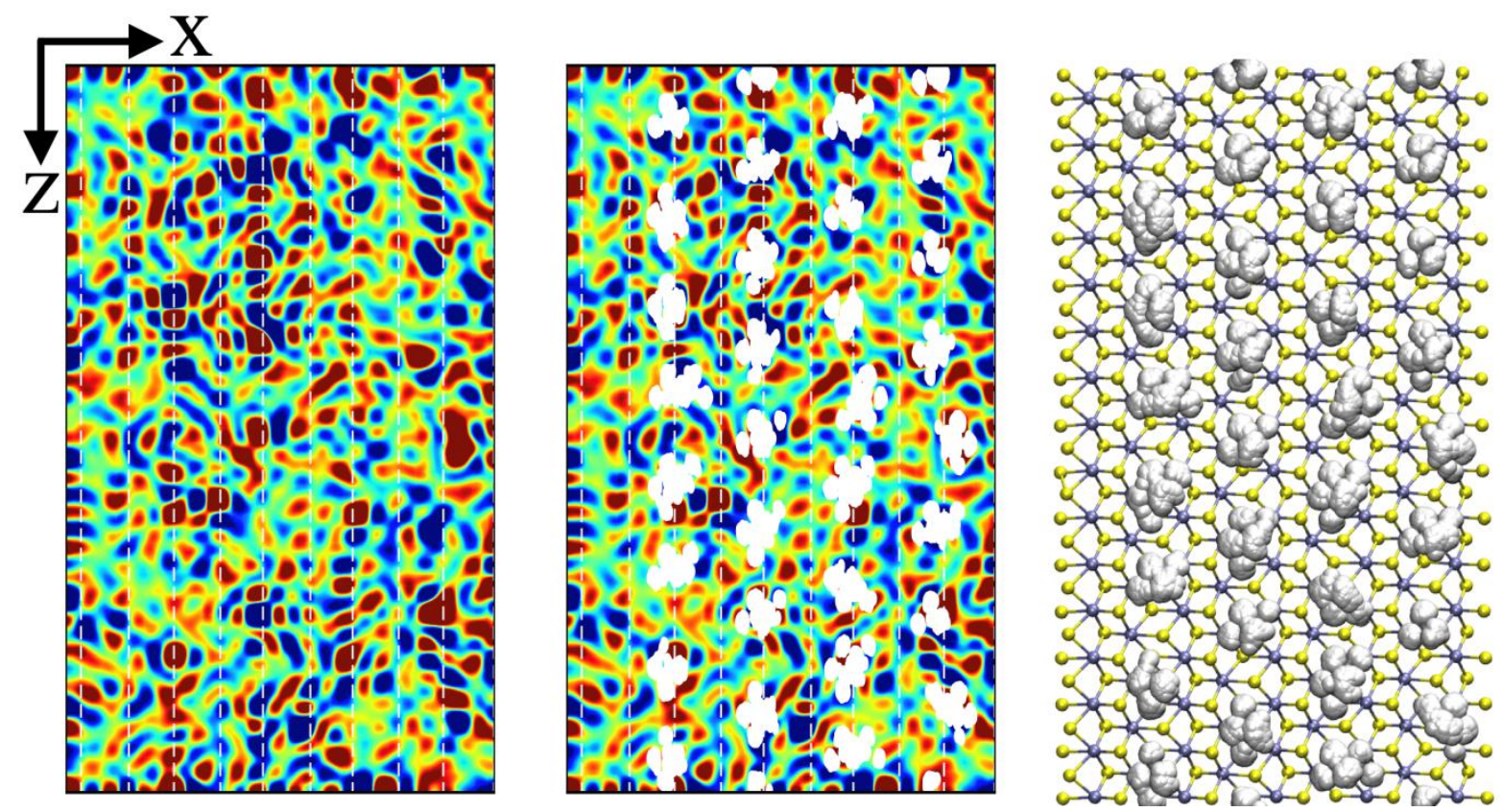

Supplementary Figure 40. From left to right, time averaged velocity map of the first layer of water molecules in the $\mathrm{XZ}$ plane of methyl-functionalized $\mathrm{MoS}_{2}$ membranes, same map including the actual position of the $\mathrm{C}$ and $\mathrm{H}$ atoms of the methyl group during the time averaged simulation, and molecular model of the layer of methyl-MoS $\mathrm{M}_{2}$ channel. Vertical white dashed lines correspond to the location of $\mathrm{S}$ atoms along the flux direction..

Indeed, we can see from main Figure 4c that the top and bottom S-atoms of the channel are slightly shifted in $\mathrm{x}$. The velocity of each water molecule of the selected layer are averaged into $1 \AA^{2}$ bins along the $1 \mathrm{~ns}$ trajectory and interpolated for better clarity. The velocity maps and their projection along the $\mathrm{x}$-axis are represented in Supplementary Figure 41 together with the position of the nearest $\mathrm{S}$-atoms of the $\mathrm{MoS}_{2}$ layer as white (map) and black (projection) dashed lines. Because of the limited amount of data (1 ns), the averaged velocities are quite large however, we can clearly see from the maps the higher mobility of water molecules in contact with methyl-functionalized $\mathrm{MoS}_{2}$. We note that minima in the velocity projection along the pristine channel are aligned with the 
location of S-atoms. By contrast, maxima in the velocity projection along methylfunctionalized channel are aligned with S-atoms. From this, we conclude that S atoms represent "pinning points" between highly polarized Mo-S and O-H bonds of electrostatic nature. The presence of methyl groups substitutes some of these pinning points and enhances the velocity of water molecules located below them. These results thus predict that the hydrophobic nature of methyl functionalized membranes is responsible for the enhanced water flux.
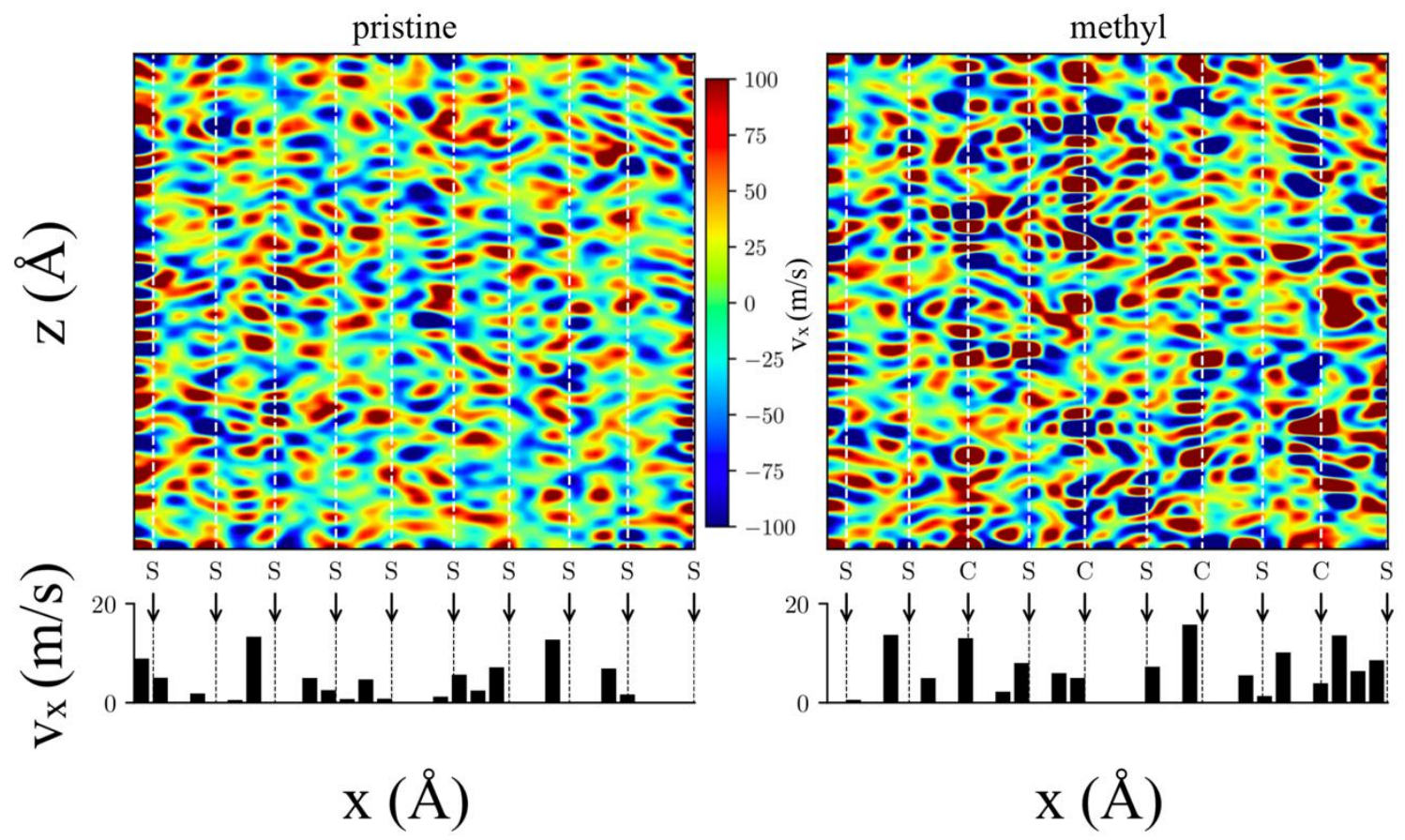

Supplementary Figure 41. Time averaged velocity map of the first layer of water molecules in the $\mathrm{XZ}$ plane and its projection along the channel direction. The velocity of water molecules is enhanced by the presence of methyl groups.

\section{Size effect of functional groups}

Since the ADF is normalized to the average number of water molecules in the channel, we can see from the area under the curve that much less water molecules are present 
between acetamide and ethyl-2-ol functionalized channels. This can be attributed to steric effects of the large functional groups. Integrating the curves gives 292, 256, 104 and 123 water molecules for pristine (non-functionalized), methyl, acetamide and ethyl-2-ol functionalized $\mathrm{MoS}_{2}$ channels. Additionally, we show Supplementary Figure 42 the position of oxygen and hydrogen atoms from water in the channels as red and gray dots, respectively, accumulated over 1,000 MD frames (=500 ps) of the simulations presented Figure 4f. We can see that water molecules in pristine and methyl-functionalized $\mathrm{MoS}_{2}$ explored most of the volume available in the nanochannel. By contrast, water in acetamide and ethyl-2-ol functionalized $\mathrm{MoS}_{2}$ are segregated in various regions of the channel. This confirms the steric effect of large functional groups and their limitation to water flow.
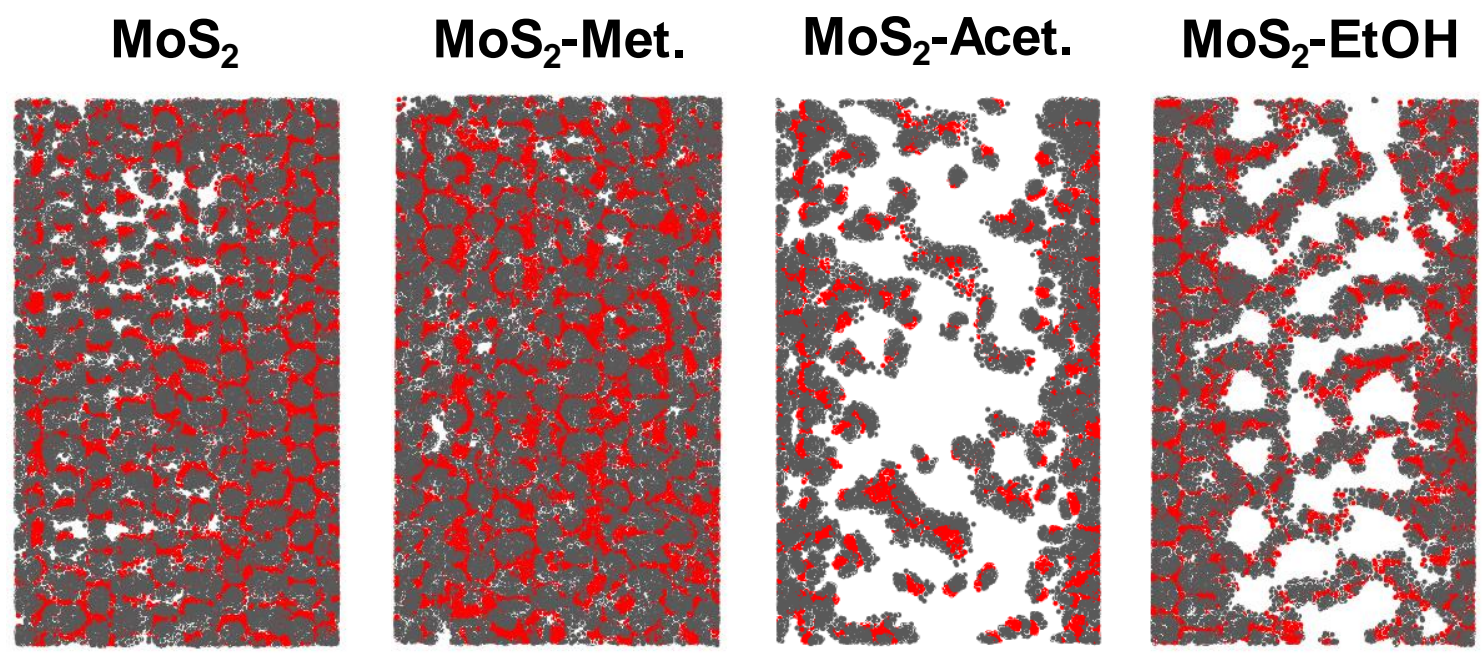

Supplementary Figure 42. Position of oxygen (red) and hydrogen (gray) atoms in pristine, methyl, acetamide and ethyl-2-ol channels accumulated during 500 ps of nanofluidic simulation. 


\section{Concentration of the functional groups.}

Since S-atoms represent pinning points for water and that the methyl-functionalization is assumed to enhance water mobility, the flux should be directly proportional to the fraction of functionalized S-atoms. We performed nanofluidic simulations, similar to those presented section "Nanofluidic simulations", for 12, 25 and $50 \%$ methylfunctionalized $\mathrm{MoS}_{2}$ channels. Supplementary Figure 43 shows the corresponding number of water molecules crossing the channel as a function of simulation time. We found fluxes of $6.2,15.7$ and $31.1 \mathrm{~L}^{-1} \mathrm{~h}^{-1} \cdot \mathrm{m}^{-2} \cdot \mathrm{bar}^{-1}$ for 12,25 and $50 \%$ methylfunctionalized $\mathrm{MoS}_{2}$ channels, respectively. The near-perfect correlation between fluxes and the fraction of functionalized S-atoms demonstrates the surface mechanism of functional group to enhance water flow.

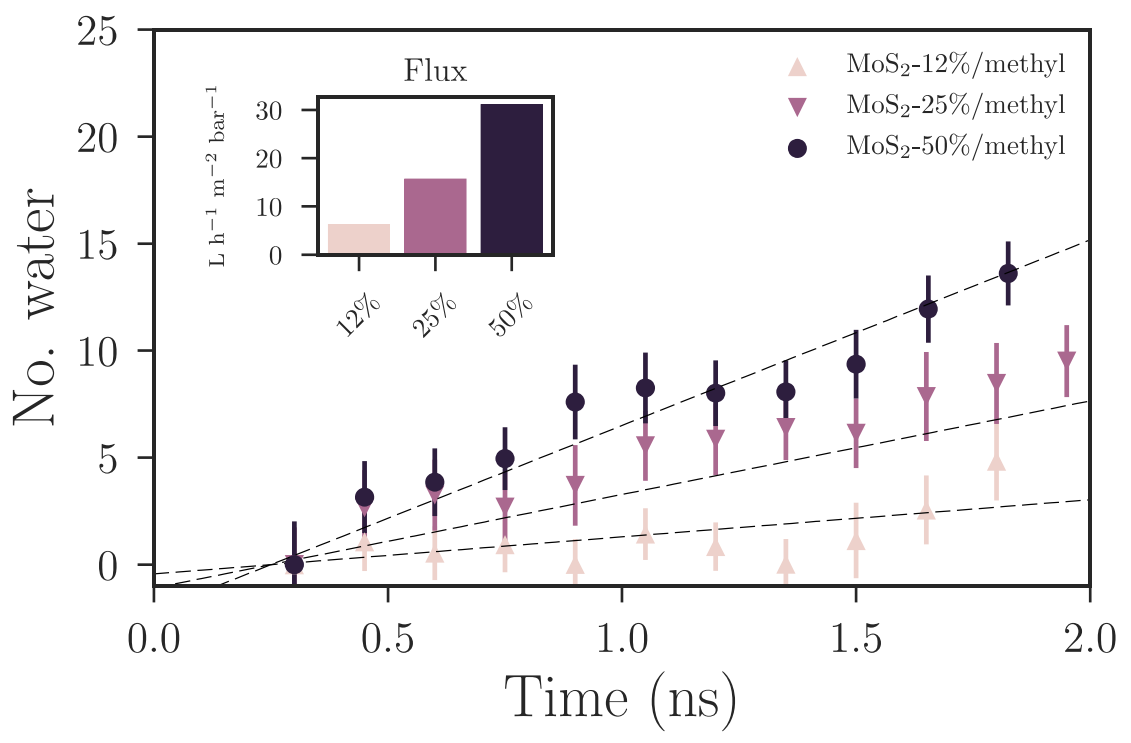

Supplementary Figure 43. Number of water molecules crossing the mid-point of the nanochannel as a function of simulation time for 12, 25 and $50 \%$ methyl-functionalized $\mathrm{MoS}_{2}$. The corresponding water fluxes are summarized in the inset. 


\section{References}

1. Heising, J. \& Kanatzidis, M. G. Structure of Restacked $\mathrm{MoS}_{2}$ and $\mathrm{WS}_{2}$ Elucidated by Electron Crystallography. J. Am. Chem. Soc. 121, 638-643 (1999).

2. Voiry, D. et al. The role of electronic coupling between substrate and 2D MoS2 nanosheets in electrocatalytic production of hydrogen. Nat. Mater. 15, 1003-1009 (2016).

3. Chow, P. K. et al. Wetting of Mono and Few-Layered $\mathrm{WS}_{2}$ and $\mathrm{MoS}_{2}$ Films Supported on $\mathrm{Si} / \mathrm{SiO}_{2}$ Substrates. ACS Nano 9, 3023-3031 (2015).

4. Heising, J. \& Kanatzidis, M. G. Exfoliated and Restacked $\mathrm{MoS}_{2}$ and $\mathrm{WS}_{2}$ : Ionic or Neutral Species? Encapsulation and Ordering of Hard Electropositive Cations. J. Am. Chem. Soc. 121, 11720-11732 (1999).

5. Hirunpinyopas, W. et al. Desalination and Nanofiltration through Functionalized Laminar $\mathrm{MoS}_{2}$ Membranes. ACS Nano 11, 11082-11090 (2017).

6. Hirata, M., Gotou, T., Horiuchi, S., Fujiwara, M. \& Ohba, M. Thin-film particles of graphite oxide 1: Carbon 42, 2929-2937 (2004).

7. Voiry, D. et al. Covalent functionalization of monolayered transition metal dichalcogenides by phase engineering. Nat. Chem. 7, 45-49 (2015).

8. Shen, Y., Wang, H., Zhang, X. \& Zhang, Y. $\operatorname{MoS}_{2}$ Nanosheets Functionalized Composite Mixed Matrix Membrane for Enhanced $\mathrm{CO}_{2}$ Capture via Surface DropCoating Method. ACS Appl. Mater. Interfaces 8, 23371-23378 (2016).

9. Calandra, M. Chemically exfoliated single-layer MoS 2 : Stability, lattice dynamics, and catalytic adsorption from first principles. Phys. Rev. B 88, (2013). 
10. Abraham, J. et al. Tunable sieving of ions using graphene oxide membranes. Nat. Nanotechnol. 12, 546-550 (2017).

11. Joshi, R. K. et al. Precise and Ultrafast Molecular Sieving Through Graphene Oxide Membranes. Science 343, 752-754 (2014).

12. Varrla, E. et al. Large-Scale Production of Size-Controlled $\mathrm{MoS}_{2}$ Nanosheets by Shear Exfoliation. Chem. Mater. 27, 1129-1139 (2015).

13. Neese, F. The ORCA program system: The ORCA program system. Wiley Interdiscip. Rev. Comput. Mol. Sci. 2, 73-78 (2012).

14. Han, Y., Xu, Z. \& Gao, C. Ultrathin graphene nanofiltration membrane for water purification. Adv. Funct. Mater. 23, 3693-3700 (2013).

15. Derek W. Smith. Ionic hydration enthalpies.pdf. (1977).

16. Picaud, F. et al. Biomimetic solution against dewetting in a highly hydrophobic nanopore. Soft Matter 12, 4903-4911 (2016).

17. Deng, M., Kwac, K., Li, M., Jung, Y. \& Park, H. G. Stability, Molecular Sieving, and Ion Diffusion Selectivity of a Lamellar Membrane from Two-Dimensional Molybdenum Disulfide. Nano Lett. 17, 2342-2348 (2017).

18. Chen, L. et al. Ion sieving in graphene oxide membranes via cationic control of interlayer spacing. Nature 550, 380 (2017).

19. Suk, M. E. \& Aluru, N. R. Water Transport through Ultrathin Graphene. J. Phys. Chem. Lett. 1, 1590-1594 (2010).

20. Chong, J. Y., Wang, B., Mattevi, C. \& Li, K. Dynamic microstructure of graphene oxide membranes and the permeation flux. J. Membr. Sci. 549, 385-392 (2018). 
21. Xu, F., Song, Y., Wei, M. \& Wang, Y. Water Flow through Interlayer Channels of Two-Dimensional Materials with Various Hydrophilicities. J. Phys. Chem. C 122, 15772-15779 (2018).

22. Kang, B. et al. Catalytically active supramolecular porphyrin boxes: acceleration of the methanolysis of phosphate triesters via a combination of increased local nucleophilicity and reactant encapsulation. Chem. Sci. 3, 1938 (2012).

23. Price, W. E. Tracer caffeine diffusion in aqueous solutions at $298 \mathrm{~K}$. The effect of caffeine self-association. J. Chem. Soc. Faraday Trans. 1 Phys. Chem. Condens. Phases 85, 415-419 (1989).

24. Ribeiro, A. C. F. et al. Diffusion coefficients of paracetamol in aqueous solutions. $J$. Chem. Thermodyn. 54, 97-99 (2012).

25. Drug Design: Medicinal Chemistry: A Series of Monographs, Volume 4.

26. Yang, Q. et al. Ultrathin graphene-based membrane with precise molecular sieving and ultrafast solvent permeation. Nat. Mater. 16, 1198 (2017).

27. Sun, L., Huang, H. \& Peng, X. Laminar MoS2 membranes for molecule separation. Chem. Commun. 49, 10718 (2013).

28. Hu, M. \& Mi, B. Enabling Graphene Oxide Nanosheets as Water Separation Membranes. Environ. Sci. Technol. 47, 3715-3723 (2013).

29. Han, Y., Jiang, Y. \& Gao, C. High-Flux Graphene Oxide Nanofiltration Membrane Intercalated by Carbon Nanotubes. ACS Appl. Mater. Interfaces 7, 8147-8155 (2015). 
30. Wang, N., Ji, S., Zhang, G., Li, J. \& Wang, L. Self-assembly of graphene oxide and polyelectrolyte complex nanohybrid membranes for nanofiltration and pervaporation. Chem. Eng. J. 213, 318-329 (2012).

31. Morelos-Gomez, A. et al. Effective $\mathrm{NaCl}$ and dye rejection of hybrid graphene oxide/graphene layered membranes. Nat. Nanotechnol. 12, 1083-1088 (2017).

32. Geise, G. M., Park, H. B., Sagle, A. C., Freeman, B. D. \& McGrath, J. E. Water permeability and water/salt selectivity tradeoff in polymers for desalination. $J$. Membr. Sci. 369, 130-138 (2011).

33. Veolia, B. W. E., MBROYN307130. RO MEMBRANES VRO-SE4040. (2016).

34. Veolia, S. W. E. M. RO MEMBRANES VRO-400-28. (2016).

35. Brackish Water element BW30-440i High Surface Area - Dow FILMTEC ${ }^{\mathrm{TM}}$ Membranes - Form No. 609-00204-1108.

36. Sea Water element SW30XHR-400i- Dow FILMTEC ${ }^{\mathrm{TM}}$ Membranes - Form No. 60900204-1108.

37. Sea Water element SW30ULE-400i - Dow FILMTEC ${ }^{\mathrm{TM}}$ Membranes - Form No. 609-00204-1108.

38. Sea Water element SW30XLE-400i - Dow FILMTEC ${ }^{\mathrm{TM}}$ Membranes - Form No. 609-00204-1108.

39. Sea Water element SW30HR LE-400i - Dow FILMTEC ${ }^{\mathrm{TM}}$ Membranes - Form No. 609-00204-1108.

40. Sea Water element SW30HR LE-400- Dow FILMTEC ${ }^{\mathrm{TM}}$ Membranes - Form No. 609-00204-1108. 
41. Sea Water element SW30HR-370/34i- Dow FILMTEC ${ }^{\mathrm{TM}}$ Membranes - Form No. 609-00204-1108.

42. Sea Water element SW30HR-380- Dow FILMTEC ${ }^{\mathrm{TM}}$ Membranes - Form No. 60900204-1108.

43. Zhao, G., Hu, R., Zhao, X., He, Y. \& Zhu, H. High flux nanofiltration membranes prepared with a graphene oxide homo-structure. J. Membr. Sci. 585, 29-37 (2019).

44. van Duin, A. C. T., Dasgupta, S., Lorant, F. \& Goddard, W. A. ReaxFF: A Reactive Force Field for Hydrocarbons. J. Phys. Chem. A 105, 9396-9409 (2001).

45. Kim, S.-Y. \& van Duin, A. C. T. Simulation of Titanium Metal/Titanium Dioxide Etching with Chlorine and Hydrogen Chloride Gases Using the ReaxFF Reactive Force Field. J. Phys. Chem. A 117, 5655-5663 (2013).

46. Ostadhossein, A. et al. ReaxFF Reactive Force-Field Study of Molybdenum Disulfide $\left(\mathrm{MoS}_{2}\right)$. J. Phys. Chem. Lett. 8, 631-640 (2017).

47. Chenoweth, K., van Duin, A. C. T. \& Goddard, W. A. The ReaxFF Monte Carlo Reactive Dynamics Method for Predicting Atomistic Structures of Disordered Ceramics: Application to the Mo3VOx Catalyst. Angew. Chem. Int. Ed. 48, 76307634 (2009).

48. Ingebrigtsen, T. \& Toxvaerd, S. Contact Angles of Lennard-Jones Liquids and Droplets on Planar Surfaces. J. Phys. Chem. C 111, 8518-8523 (2007).

49. Thomas, J. A. \& McGaughey, A. J. H. Water Flow in Carbon Nanotubes: Transition to Subcontinuum Transport. Phys. Rev. Lett. 102, (2009). 
50. Sresht, V. et al. Quantitative Modeling of $\mathrm{MoS}_{2}$-Solvent Interfaces: Predicting Contact Angles and Exfoliation Performance using Molecular Dynamics. J. Phys. Chem. C 121, 9022-9031 (2017).

51. Kresse, G. \& Furthmüller, J. Efficiency of ab-initio total energy calculations for metals and semiconductors using a plane-wave basis set. Comput. Mater. Sci. 6, 1550 (1996).

52. Kresse, G. \& Furthmüller, J. Efficient iterative schemes for ab initio total-energy calculations using a plane-wave basis set. Phys. Rev. B 54, 11169-11186 (1996).

53. Perdew, J. P., Burke, K. \& Ernzerhof, M. Generalized Gradient Approximation Made Simple. Phys. Rev. Lett. 77, 3865-3868 (1996).

54. Grimme, S. Semiempirical GGA-type density functional constructed with a longrange dispersion correction. J. Comput. Chem. 27, 1787-1799 (2006).

55. Berendsen, H. J. C., Postma, J. P. M., van Gunsteren, W. F., DiNola, A. \& Haak, J. R. Molecular dynamics with coupling to an external bath. J. Chem. Phys. 81, 36843690 (1984).

56. Kwac, K. et al. Multilayer Two-Dimensional Water Structure Confined in MoS $2 . J$. Phys. Chem. C 121, 16021-16028 (2017).

57. Grdadolnik, J., Merzel, F. \& Avbelj, F. Origin of hydrophobicity and enhanced water hydrogen bond strength near purely hydrophobic solutes. Proc. Natl. Acad. Sci. 114, 322-327 (2017). 